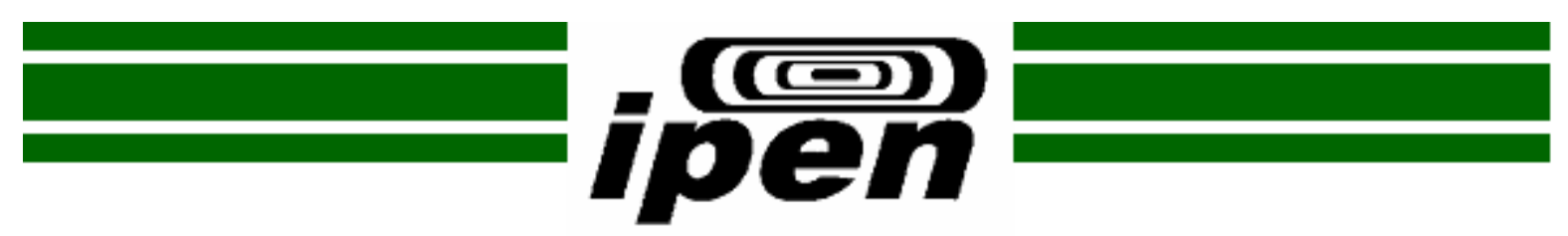

Autarquia associada à Universidade de São Paulo

\title{
AVALIAÇÃO DOS CRITÉRIOS DE QUALIDADE DE IMAGEM E ESTUDO DAS DOSES EM UM DEPARTAMENTO DE MAMOGRAFIA
}

\author{
Marcela Costa Alcântara
}

\begin{abstract}
Dissertação apresentada como parte dos requisitos para obtenção do Grau de Mestre em Ciências na Área de Tecnologia Nuclear - Aplicações.
\end{abstract}

Orientador:

Doutor Gian-Maria A. A. Sordi

SÃo PAULO 
INSTITUTO DE PESQUISAS ENERGÉTICAS E NUCLEARES

Autarquia associada à Universidade de São Paulo

\section{AVALIAÇÃO DOS CRITÉRIOS DE QUALIDADE DE IMAGEM E ESTUDO DAS DOSES EM UM DEPARTAMENTO DE MAMOGRAFIA}

Marcela Costa Alcântara

Dissertação apresentada como parte dos requisitos para obtenção do Grau de Mestre em Ciências na Área de Tecnologia Nuclear - Aplicações.

Orientador:

Doutor Gian-Maria A. A. Sordi

SÃO PAULO 
À toda a minha família. 


\section{AGRADECIMENTOS}

Ao IPEN, pela oportunidade de desenvolver este trabalho.

Ao Dr. Gian-Maria A. A. Sordi pela orientação, compreensão e ensinamentos que levarei comigo para sempre.

À Dra. Tânia Furquim pela imensa colaboração em todo o projeto e por todas as conversas amigas. Serei eternamente grata pela sua dedicação e empenho em todas as etapas deste trabalho.

À Dra. Linda, pelos ensinamentos, paciência e acolhimento desde o início da minha estadia em São Paulo.

À Donata Zanin, pela amizade e grande auxílio em assuntos burocráticos.

Ao Instituto de Radiologia do Hospital das Clínicas, por permitir o desenvolvimento deste projeto e a toda equipe de mamografia, principalmente às funcionárias, técnicas em radiologia, pela colaboração e por todo o carinho que me foi dado durante os 12 meses que acompanhei este serviço.

Aos meus pais, Geraldo e Fátima, que compreenderam a minha ausência física para a busca do conhecimento e por todo amor e carinho que me proporcionam.

Aos meus irmãos, Gegeu e Isa, pelos momentos felizes que ajudaram a encurtar a distância.

Ao meu amor, Juliano, por estar ao meu lado em todos os momentos difíceis, pelo esforço em ajudar e deixar tudo tão fácil, dando forças para que eu pudesse seguir em frente.

Ao Lucas, pelos momentos de diversão e apesar da pouca idade, por entender a minha necessidade de dedicação intensa ao mestrado.

Às minhas avós, Joseline e Lourdinha, e meu avô, Geraldo, por todo apoio e palavras carinhosas, mesmo estando distantes. Sei que meu avô Dermeval (in memorian) também torce pelo meu sucesso, esteja onde estiver. 
Às minhas tias amadas e meus tios queridos, por acreditarem que eu iria conseguir e por darem coragem e força para continuar, superando a saudade de todos.

À todos os meus primos e primas por todo o apoio e companhia que me proporcionaram ao virem me visitar.

Às minhas companheiras de apartamento, Nandra e Ana Paula, e à minha ex vizinha Antônia, pelas conversas animadoras, paciência e companheirismo ao longo de todo esse tempo.

Às amigas que São Paulo me trouxe, Vera, Gisene, Raquel e Dalila, por todo o auxílio, companhia e aventuras.

À toda a família Amadeu, por me acolher e proporcionar bons momentos, principalmente aos domingos.

À Leila de Paula, pela compreensão, oportunidades e ensinamentos que levarei comigo para sempre. Ao Paulo Costa, pelo incentivo e ajuda oferecida para a realização deste trabalho. Ao casal pelos momentos de descontração nos jantares nas sextas-feiras.

Ao meu amigo Daniel, futuro companheiro de trabalho, pela troca de conhecimento e experiência e às minhas amigas de Aracaju, Marcella, Heline, Carol, Maíra, Manuela, Juliana, Rosana, Ludmilla e Gil, que mesmo distantes, sempre se mantiveram perto.

Aos colegas do IPEN, por todo auxílio e momentos de descontração ao longo desse tempo e ao Professor Goro pelas orientações no estudo estatístico.

Aos colegas do IEE, principalmente à equipe da STADI, pela amizade, pelo conhecimento que foi fornecido para mim e por serem tão receptivos e solidários.

À Comissão Nacional de Energia Nuclear (CNEN), Conselho Nacional do Desenvolvimento Científico e Tecnológico (CNPq) e Fundação de Amparo do Estado de São Paulo (FAPESP) pelo auxílio financeiro que permitiu o desenvolvimento deste projeto. 


\title{
AVALIAÇÃO DOS CRITÉRIOS DE QUALIDADE DE IMAGEM E ESTUDO DAS DOSES EM UM DEPARTAMENTO DE MAMOGRAFIA
}

\author{
Marcela Costa Alcântara
}

\begin{abstract}
RESUMO
Os critérios de qualidade de imagem mamógrafica publicados pela European Commission foram implementados em três mamógrafos de um mesmo departamento de radiologia de um hospital na cidade de São Paulo. Dentre os mamógrafos dois apresentam o sistema tela-filme e um deles apresenta o sistema digital indireto. Durante o período de coleta de dados, foi observada a necessidade de realizar um estudo sobre índice de rejeição de imagem em cada mamógrafo. Portanto, este estudo foi realizado e, em seguida, foram feitas comparações, entre os mamógrafos, do índice de rejeição de imagem e da porcentagem de imagens que atendiam a cada critério de qualidade de imagem. Paralelamente a esses estudos, foi realizado o estudo das doses na entrada da pele e glandular média. Essas doses foram estimadas, baseando-se em diferentes metodologias apresentadas por diferentes grupos de estudiosos, para todas as combinações anodo-filtro apresentadas pelo equipamento. Para estimar a dose na entrada da pele pelo método publicado no guia da ANVISA e a dose glandular média pelo método de $\mathrm{Wu}$, foi desenvolvido um manequim no formato bem próximo ao de uma mama, em diferentes espessuras de PMMA. Por fim, associou-se a qualidade da imagem com a dose recebida pela paciente. $O$ equipamento digital apresentou melhores resultados na avaliação dos critérios de qualidade, menor índice de rejeição de imagem e menores valores de dose glandular média e na entrada da pele em todos os métodos estudados. Porém não é suficiente, pois não atende às pacientes que possuem mamas grandes.
\end{abstract}




\title{
EVALUATION OF THE IMAGE QUALITY CRITERIA AND STUDY OF DOSIS IN A MAMMOGRAPHY DEPARTMENT
}

\author{
Marcela Costa Alcântara
}

\begin{abstract}
The mammographic image quality criteria published by European Commission were implemented in three mammography equipments of a same radiology department in a hospital of Sao Paulo city. Among the mammography equipments, two use the screen-film system and one of them uses the indirect digital system. During the data collection, it was noted the need to conduct a study about image rejection in each mammography equipment. Therefore, this study was realized and, after that, the results in each mammography equipment of image rejection and image percentage that present each quality criterion it were compared. At the same time of this studies, it was realized other study about surface entrance dose and average glandular dose. These doses it was estimated based on different methods published by different groups of researcher, for all combinations anodefilter available in the equipment. To estimate the surface entrance dose following the methodology published in ANVISA' guide and the average glandular dose following the W' methodology, it was developed a phantom, in different thicknesses of acrylic, to simulate a breast. Finally, the image quality it was associated with the dose received by patient. The digital equipment shows better results in the evaluation of quality criteria, lower rate of image rejection and lower values of average glandular dose and surface entrance dose in all methods studied. But it is not sufficient, because is not adequate for patients with great breast.
\end{abstract}




\section{SUMÁRIO}

Página

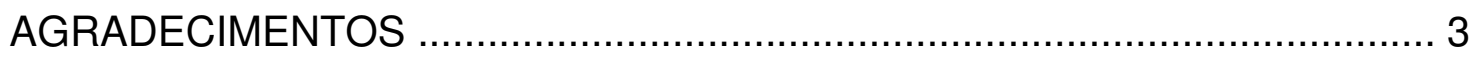

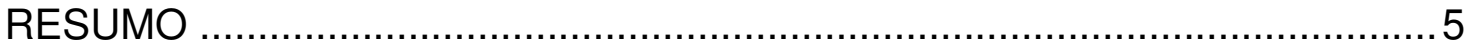

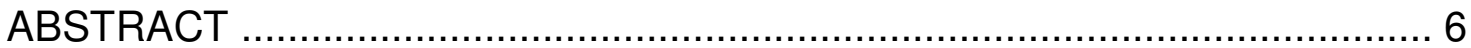

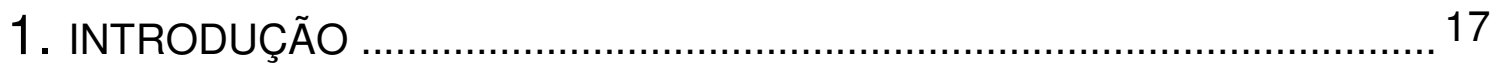

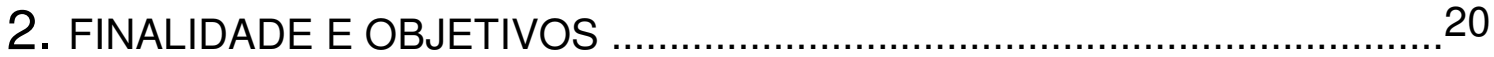

2.1. Finalidade 20

2.2. Objetivos 20

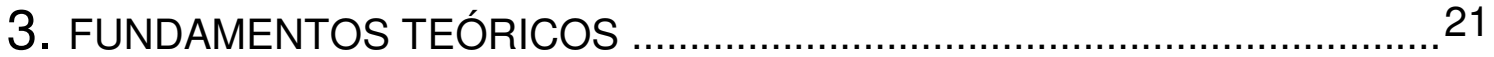

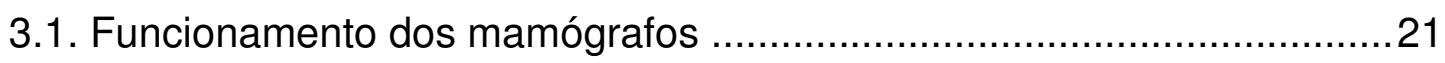

3.2. Controle de qualidade nos mamógrafos ............................................ 25

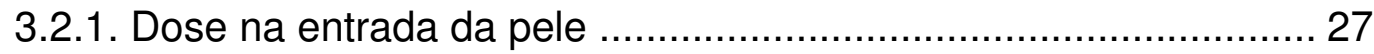

3.2.1.1. Método do Guia da ANVISA ........................................27

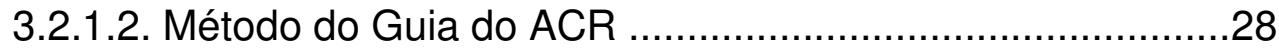

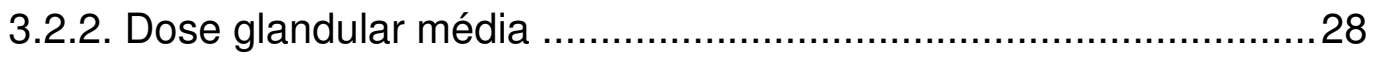

3.2.2.1. Método de Dance ....................................................... 29

3.2.2.2. Método de Wu ............................................................. 32

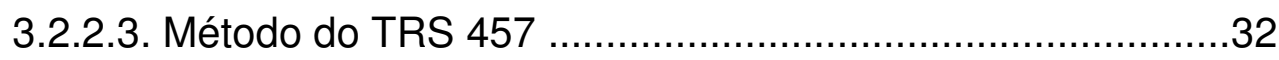

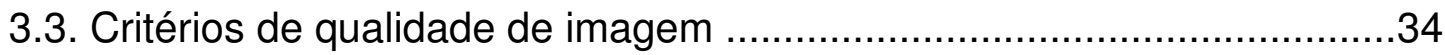

4. EQUIPAMENTOS, MATERIAIS E METODOLOGIA ................................ 36

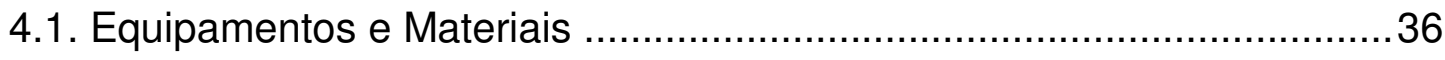

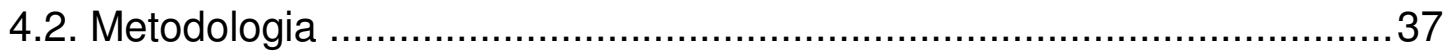

4.2.1. Determinação da dimensão da amostra.....................................37

4.2.2. Critérios de qualidade de imagem ............................................ 38

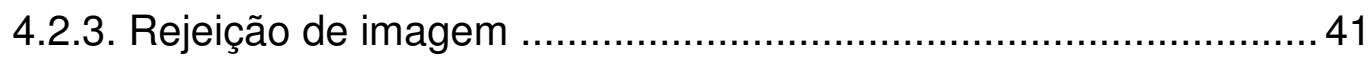

4.2.4. Estimativa da dose na entrada da pele e da dose glandular 41 média

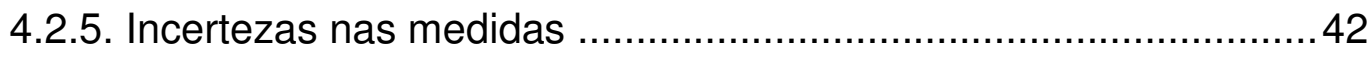

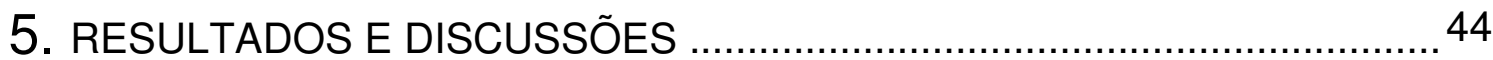


5.1. Controle de qualidade nos mamógrafos................................................ 44

5.2. Critérios de qualidade de imagem ...................................................... 49

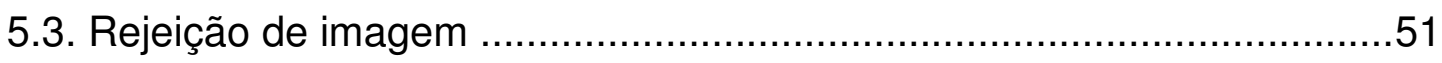

5.4. Estimativa da dose na entrada da pele e da dose glandular média .......54

5.4.1. Desenvolvimento do manequim ............................................. 54

5.4.2. Determinação da camada semi-redutora..................................... 54

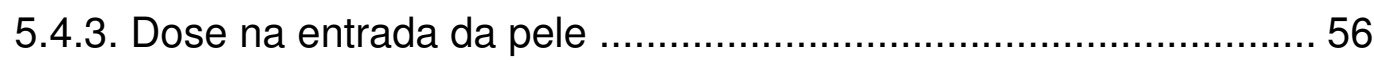

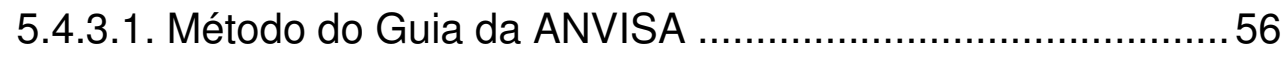

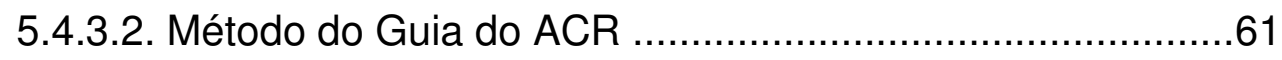

5.4.3.3. Comparação entre os métodos ...................................... 65

5.4.3.4. Comparação entre os mamógrafos …………................... 70

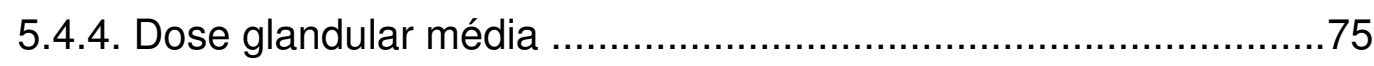

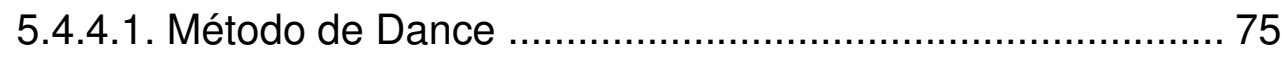

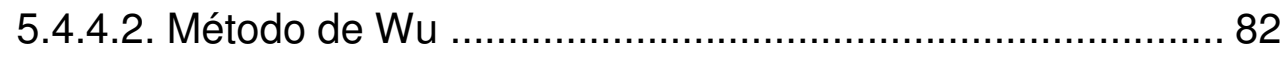

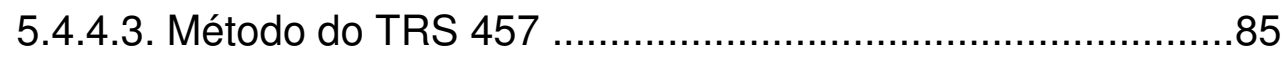

5.4.4.4. Comparação entre os métodos ....................................... 91

5.4.4.5. Comparação entre os mamógrafos ................................... 102

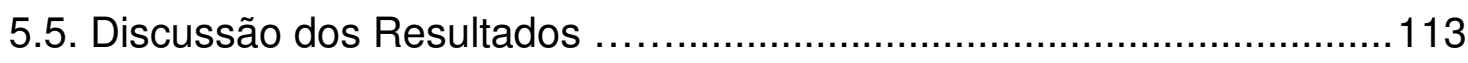

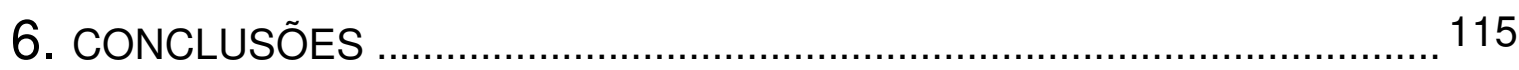

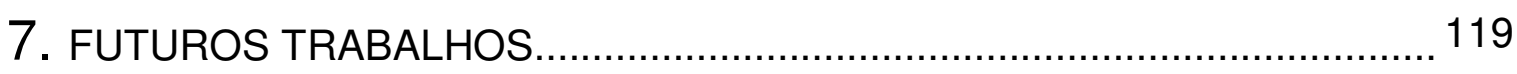

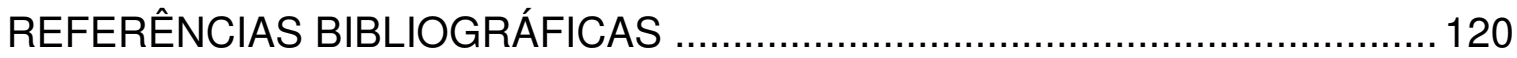




\section{LISTA DE TABELAS}

Página

Tabela 1 Fatores de retro-espalhamento para diferentes valores de camada semiredutora determinados por Kramer e colaboradores ${ }^{15}$

Tabela 2 Coeficientes para o ajuste polinomial da glandularidade em função da espessura da mama

Tabela 3 Fatores de conversão $\mathrm{g}$ ( $\mathrm{mGy} / \mathrm{mGy}$ ) para espessuras de mama de 2 a $11 \mathrm{~cm}$ e CSR entre 0,30 a $0,60 \mathrm{mmAl}$

Tabela 4 Fatores de conversão c para glandularidades de 0,1 a $100 \%$ na região central da mama, espessuras de mama de 2 a $11 \mathrm{~cm}$ e CSR de 0,30 a 0,60 mmAl

Tabela 5 Fatores de conversão s do espectro utilizados clinicamente e erros Máximos que podem ocorrer quando são utilizados

Tabela 6 Tabela utilizada na coleta de dados dos exames na projeção Crânio

Caudal

Tabela 7 Tabela utilizada na coleta de dados dos exames na projeção Médio Lateral Oblíqua

Tabela 8 Resultados dos testes de controle de qualidade realizados nos três mamógrafos.

Tabela 9 Principais causas de rejeição de imagem

Tabela 10 Valores de camada semi-redutora (mmAl) para cada tensão de aceleração de elétrons $(k V p)$ calculados nas diferentes combinações anodo-filtro nos três mamógrafos estudados

Tabela 11 Diferença de DEP (\%) estimada pelo método do ACR para uma mesma espessura de mama comprimida e para uma mesma paciente para as diferentes combinações anodo-filtro nos três mamógrafos 


\section{LISTA DE FIGURAS}

\section{Página}

Figura 1 Evolução dos tipos de câncer mais incidentes no sexo feminino de 2005 para 2008

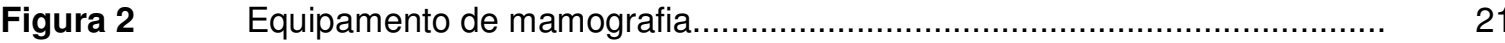

Figura $3 \quad$ Geometria do tubo de raios X mamográfico ........................................ 22

Figura $4 \quad$ Espectro de energia dos raios $X$ para as combinações anodo-filtro (a)

Mo/Mo, (b) W/Rh, (c) W/Mo, (d) Rh/Rh e (e) Mo/Rh

Figura 5 Diferentes projeções da mama que podem ser obtidas em um exame de mamografia

Figura 6 Equipamentos de mamografia estudados: A. GE Senographe 700T.

B. Siemens Mammomat 3000 Nova. C. GE Senographe DS

Figura 7 Instrumentos para a realização das medições. A. Conjunto Radcal composto por câmara de ionização de 6 cc, monitor, medidor e sensor de tensão e tempo de exposição. B. Filtros de alumínio da Nuclear Associates. C. Manequim de PMMA para diferentes espessuras

Figura $8 \quad$ Porcentual dos exames que atendem a cada critério de qualidade na projeção (a) CC e (b) MLO nos três mamógrafos

Figura 9 Comparação entre os valores de CSR obtidos para cada tensão de aceleração de elétrons (kVp) nas diferentes combinações anodo-filtro em cada mamógrafo

Figura 10 Dose na entrada da pele para cada espessura de mama comprimida estudada na projeção CC e MLO no equipamento GE $700 \mathrm{~T}$

Figura 11 Dose na entrada da pele para cada espessura de mama comprimida estudada na projeção CC e MLO no equipamento Siemens, combinação Mo/Mo

Figura 12 Dose na entrada da pele para cada espessura de mama comprimida estudada na projeção CC e MLO no equipamento Siemens, combinação Mo/Rh

Figura 13 Dose na entrada da pele para cada espessura de mama comprimida estudada na projeção CC e MLO no equipamento GE DS, combinação Mo/Rh

Figura 14 Dose na entrada da pele para cada espessura de mama comprimida estudada da projeção CC e MLO no equipamento GE DS, combinação Rh/Rh

Figura $15 \quad$ Dose na entrada da pele para cada espessura de mama comprimida estudada da projeção CC e MLO no equipamento GE 700 T

Figura 16 Dose na entrada da pele para cada espessura de mama comprimida estudada na projeção CC e MLO no equipamento Siemens - MoMo

Figura 17 Dose na entrada da pele para cada espessura de mama comprimida estudada da projeção CC e MLO no equipamento Siemens - MoRh

Figura 18 Dose na entrada da pele para cada espessura de mama comprimida estudada da projeção CC e MLO no equipamento GE DS - MoRh 
Figura 19 Dose na entrada da pele para cada espessura de mama comprimida estudada da projeção CC e MLO no equipamento GE DS - RhRh

Figura 20 Comparação entre as estimativas de DEP calculada pelos dois métodos diferentes para cada espessura de mama comprimida estudada na projeção CC no equipamento GE $700 \mathrm{~T}$

Figura 21 Comparação entre as estimativas de DEP calculada pelos dois métodos diferentes para cada espessura de mama comprimida estudada na projeção MLO no equipamento GE $700 \mathrm{~T}$

Figura 22 Comparação entre as estimativas de DEP calculada pelos dois métodos diferentes para cada espessura de mama comprimida estudada na projeção CC no equipamento Siemens - MoMo

Figura 23 Comparação entre as estimativas de DEP calculada pelos dois métodos diferentes para cada espessura de mama comprimida estudada na projeção MLO no equipamento Siemens - MoMo

Figura 24 Comparação entre as estimativas de DEP calculada pelos dois métodos diferentes para cada espessura de mama comprimida estudada na projeção CC no equipamento Siemens - MoRh

Figura 25 Comparação entre as estimativas de DEP calculada pelos dois métodos diferentes para cada espessura de mama comprimida estudada na projeção MLO no equipamento Siemens - MoRh

Figura 26 Comparação entre as estimativas de DEP calculada pelos dois métodos diferentes para cada espessura de mama comprimida estudada na projeção CC no equipamento GE DS - MoRh

Figura 27 Comparação entre as estimativas de DEP calculada pelos dois métodos diferentes para cada espessura de mama comprimida estudada na projeção MLO no equipamento GE DS - MoRh

Figura 28 Comparação entre as estimativas de DEP calculada pelos dois métodos diferentes para cada espessura de mama comprimida estudada na projeção CC no equipamento GE DS - RhRh

Figura 29 Comparação entre as estimativas de DEP calculada pelos dois métodos diferentes para cada espessura de mama comprimida estudada na projeção MLO no equipamento GE DS - RhRh

Figura 30 Comparação entre as estimativas de DEP calculada pelo método do Guia da ANVISA para cada espessura de mama comprimida estudada na projeção CC nos equipamentos Siemens e GE DS - MoRh

Figura $31 \quad$ Comparação entre as estimativas de DEP calculada pelo método do Guia da ANVISA para cada espessura de mama comprimida estudada na projeção MLO nos equipamentos Siemens e GE DS - MoRh

Figura 32 Comparação entre as estimativas de DEP calculada pelo método do Guia ACR para cada espessura de mama comprimida estudada na projeção CC nos equipamentos Siemens e GE DS - MoRh

Figura 33 Comparação entre as estimativas de DEP calculada pelo método do Guia do ACR para cada espessura de mama comprimida estudada na projeção MLO nos equipamentos Siemens e GE DS - MoRh 
Figura $34 \quad$ Comparação entre as estimativas de DEP calculada pelo método do Guia da ANVISA para cada espessura de mama comprimida estudada na projeção CC nos equipamentos Siemens e GE 700 T - MoMo

Figura 35 Comparação entre as estimativas de DEP calculada pelo método do Guia da ANVISA para cada espessura de mama comprimida estudada na projeção MLO nos equipamentos Siemens e GE 700 T - MoMo

Figura 36 Comparação entre as estimativas de DEP calculada pelo método do Guia do ACR para cada espessura de mama comprimida estudada na projeção CC nos equipamentos Siemens e GE 700 T - MoMo

Figura 37 Comparação entre as estimativas de DEP calculada pelo método do Guia do ACR para cada espessura de mama comprimida estudada na projeção MLO nos equipamentos Siemens e GE 700 T - MoMo

Figura 38 Dose glandular média para cada espessura de mama comprimida estudada na projeção CC e MLO no equipamento GE $700 \mathrm{~T}$, para mulheres com idade inferior a 50 anos

Figura 39 Dose glandular média para cada espessura de mama comprimida estudada na projeção CC e MLO no equipamento GE 700 T, para mulheres com idade igual ou superior a 50 anos

Figura 40 Dose glandular média para cada espessura de mama comprimida estudada na projeção CC e MLO no equipamento Siemens - Mo/Mo, para mulheres com idade inferior a 50 anos

Figura 41 Dose glandular média para cada espessura de mama comprimida estudada na projeção CC e MLO no equipamento Siemens - Mo/Mo, para mulheres com idade igual ou superior a 50 anos

Figura 42 Dose glandular média para cada espessura de mama comprimida estudada na projeção CC e MLO no equipamento Siemens - Mo/Rh, para mulheres com idade inferior a 50 anos

Figura 43 Dose glandular média para cada espessura de mama comprimida estudada na projeção CC e MLO no equipamento Siemens - Mo/Rh, para mulheres com idade igual ou superior a 50 anos

Figura 44 Dose glandular média para cada espessura de mama comprimida estudada na projeção CC e MLO no equipamento GE DS - Mo/Rh, para mulheres com idade inferior a 50 anos

Figura 45 Dose glandular média para cada espessura de mama comprimida estudada na projeção CC e MLO no equipamento GE DS - Mo/Rh, para mulheres com idade igual ou superior a 50 anos

Figura 46 Dose glandular média para cada espessura de mama comprimida estudada na projeção CC e MLO no equipamento GE DS - Rh/Rh, para mulheres com idade inferior a 50 anos

Figura 47 Dose glandular média para cada espessura de mama comprimida estudada na projeção CC e MLO no equipamento GE DS - Rh/Rh, para mulheres com idade igual ou superior a 50 anos

Figura 48 Dose glandular média para cada espessura de mama comprimida estudada na projeção CC e MLO no equipamento GE $700 \mathrm{~T}$ 
Figura 49 Dose glandular média para cada espessura de mama comprimida estudada na projeção CC e MLO no equipamento Siemens - Mo/Mo

Figura 50 Dose glandular média para cada espessura de mama comprimida estudada na projeção CC e MLO no equipamento Siemens - Mo/Rh

Figura 51 Dose glandular média para cada espessura de mama comprimida estudada na projeção CC e MLO no equipamento GE DS - Mo/Rh

Figura 52 Dose glandular média para cada espessura de mama comprimida estudada na projeção CC e MLO no equipamento GE DS - Rh/Rh

Figura 53 Dose glandular média para cada espessura de mama comprimida estudada na projeção CC e MLO no equipamento GE $700 \mathrm{~T}$, para mulheres com idade inferior a 50 anos ......

Figura 54 Dose glandular média para cada espessura de mama comprimida estudada na projeção CC e MLO no equipamento GE 700 T, para mulheres com idade igual ou superior a 50 anos

Figura 55 Dose glandular média para cada espessura de mama comprimida estudada na projeção CC e MLO no equipamento Siemens - Mo/Mo, para mulheres com idade inferior a 50 anos

Figura 56 Dose glandular média para cada espessura de mama comprimida estudada na projeção CC e MLO no equipamento Siemens - Mo/Mo, para mulheres com idade igual ou superior a 50 anos

Figura 57 Dose glandular média para cada espessura de mama comprimida estudada na projeção CC e MLO no equipamento Siemens - Mo/Rh, para mulheres com idade inferior a 50 anos

Figura 58 Dose glandular média para cada espessura de mama comprimida estudada na projeção CC e MLO no equipamento Siemens - Mo/Rh, para mulheres com idade igual ou superior a 50 anos

Figura 59 Dose glandular média para cada espessura de mama comprimida estudada na projeção CC e MLO no equipamento GE DS - Mo/Rh, para mulheres com idade inferior a 50 anos.....

Figura 60 Dose glandular média para cada espessura de mama comprimida estudada na projeção CC e MLO no equipamento GE DS - Mo/Rh, para mulheres com idade igual ou superior a 50 anos

Figura 61 Dose glandular média para cada espessura de mama comprimida estudada na projeção CC e MLO no equipamento GE DS - Rh/Rh, para mulheres com idade inferior a 50 anos

Figura 62 Dose glandular média para cada espessura de mama comprimida estudada na projeção CC e MLO no equipamento GE DS - Rh/Rh, para mulheres com idade igual ou superior a 50 anos

Figura 63 Comparação entre a estimativa de dose glandular média calculada pelos três métodos diferentes para cada espessura de mama comprimida estudada na projeção CC no equipamento GE $700 \mathrm{~T}$, para mulheres com idade inferior a 50 anos

Figura 64 Comparação entre a estimativa de dose glandular média calculada pelos três métodos diferentes para cada espessura de mama comprimida estudada na projeção CC no equipamento GE $700 \mathrm{~T}$, para mulheres com idade igual ou superior a 50 anos .... 
Figura 65 Comparação entre a estimativa de dose glandular média calculada pelos três métodos diferentes para cada espessura de mama comprimida estudada na projeção MLO no equipamento GE $700 \mathrm{~T}$, para mulheres com idade inferior a 50 anos

Figura 66 Comparação entre a estimativa de dose glandular média calculada pelos três métodos diferentes para cada espessura de mama comprimida estudada na projeção MLO no equipamento GE $700 \mathrm{~T}$, para mulheres com idade igual ou superior a 50 anos ..

Figura $67 \quad$ Comparação entre a estimativa de dose glandular média calculada pelos três métodos diferentes para cada espessura de mama comprimida estudada na projeção CC no equipamento Siemens - Mo/Mo, para mulheres com idade inferior a 50 anos ......

Figura 68 Comparação entre a estimativa de dose glandular média calculada pelos três métodos diferentes para cada espessura de mama comprimida estudada na projeção CC no equipamento Siemens - Mo/Mo, para mulheres com idade igual ou superior a 50 anos

Figura 69 Comparação entre a estimativa de dose glandular média calculada pelos três métodos diferentes para cada espessura de mama comprimida estudada na projeção MLO no equipamento Siemens - Mo/Mo, para mulheres com idade inferior a 50 anos ....

Figura 70 Comparação entre a estimativa de dose glandular média calculada pelos três métodos diferentes para cada espessura de mama comprimida estudada na projeção MLO no equipamento Siemens - Mo/Mo, para mulheres com idade igual ou superior a 50 anos

Figura 71 Comparação entre a estimativa de dose glandular média calculada pelos três métodos diferentes para cada espessura de mama comprimida estudada na projeção $\mathrm{CC}$ no equipamento Siemens - Mo/Rh, para mulheres com idade inferior a 50 anos .......

Figura 72 Comparação entre a estimativa de dose glandular média calculada pelos três métodos diferentes para cada espessura de mama comprimida estudada na projeção CC no equipamento Siemens - Mo/Rh, para mulheres com idade igual ou superior a 50 anos

Figura 73 Comparação entre a estimativa de dose glandular média calculada pelos três métodos diferentes para cada espessura de mama comprimida estudada na projeção MLO no equipamento Siemens - Mo/Rh, para mulheres com idade inferior a 50 anos ....

Figura $74 \quad$ Comparação entre a estimativa de dose glandular média calculada pelos três métodos diferentes para cada espessura de mama comprimida estudada na projeção MLO no equipamento Siemens - Mo/Rh, para mulheres com idade igual ou superior a 50 anos

Figura 75 Comparação entre a estimativa de dose glandular média calculada pelos três métodos diferentes para cada espessura de mama comprimida estudada na projeção CC no equipamento GE DS - Mo/Rh, para mulheres com idade inferior a 50 anos

Figura 76 Comparação entre a estimativa de dose glandular média calculada pelos três métodos diferentes para cada espessura de mama comprimida estudada na projeção CC no equipamento GE DS - Mo/Rh, para mulheres com idade igual ou superior a 50 anos .....

Figura 77 Comparação entre a estimativa de dose glandular média calculada pelos três métodos diferentes para cada espessura de mama comprimida estudada na projeção MLO no equipamento GE DS - Mo/Rh, para mulheres com idade inferior a 50 anos ......... 
Figura 78 Comparação entre a estimativa de dose glandular média calculada pelos três métodos diferentes para cada espessura de mama comprimida estudada na projeção MLO no equipamento GE DS - Mo/Rh, para mulheres com idade igual ou superior a 50 anos ......

Figura 79 Comparação entre a estimativa de dose glandular média calculada pelos três métodos diferentes para cada espessura de mama comprimida estudada na projeção CC no equipamento GE DS - Rh/Rh, para mulheres com idade inferior a 50 anos

Figura 80 Comparação entre a estimativa de dose glandular média calculada pelos três métodos diferentes para cada espessura de mama comprimida estudada na projeção $\mathrm{CC}$ no equipamento GE DS - Rh/Rh, para mulheres com idade igual ou superior a 50 anos

Figura $81 \quad$ Comparação entre a estimativa de dose glandular média calculada pelos três métodos diferentes para cada espessura de mama comprimida estudada na projeção MLO no equipamento GE DS - Rh/Rh, para mulheres com idade inferior a 50 anos .......

Figura 82 Comparação entre a estimativa de dose glandular média calculada pelos três métodos diferentes para cada espessura de mama comprimida estudada na projeção MLO no equipamento GE DS - Rh/Rh, para mulheres com idade igual ou superior a 50 anos

Figura 83 Comparação entre as estimativas de DGM calculada pelo método de Dance para cada espessura de mama comprimida estudada na projeção CC nos equipamentos Siemens e GE DS - MoRh para mulheres com idade inferior a 50 anos

Figura 84 Comparação entre as estimativas de DGM calculada pelo método de Dance para cada espessura de mama comprimida estudada na projeção $C C$ nos equipamentos Siemens e GE DS - MoRh para mulheres com idade igual ou superior a 50 anos

Figura 85 Comparação entre as estimativas de DGM calculada pelo método de Dance para cada espessura de mama comprimida estudada na projeção MLO nos equipamentos Siemens e GE DS - MoRh para mulheres com idade inferior a 50 anos

Figura 86 Comparação entre as estimativas de DGM calculada pelo método de Dance para cada espessura de mama comprimida estudada na projeção MLO nos equipamentos Siemens e GE DS - MoRh para mulheres com idade igual ou superior a 50 anos

Figura 87 Comparação entre as estimativas de DGM calculada pelo método de $\mathrm{Wu}$ para cada espessura de mama comprimida estudada na projeção CC nos equipamentos Siemens e GE DS - MoRh

Figura $88 \quad$ Comparação entre as estimativas de DGM calculada pelo método de $\mathrm{Wu}$ para cada espessura de mama comprimida estudada na projeção MLO nos equipamentos Siemens e GE DS - MoRh

Figura 89 Comparação entre as estimativas de DGM calculada pelo método do TRS 457 para cada espessura de mama comprimida estudada na projeção CC nos equipamentos Siemens e GE DS - MoRh para mulheres com idade inferior a 50 anos

Figura 90 Comparação entre as estimativas de DGM calculada pelo método do TRS 457 para cada espessura de mama comprimida estudada na projeção CC nos equipamentos Siemens e GE DS - MoRh para mulheres com idade igual ou superior a 50 anos

Figura 91 Comparação entre as estimativas de DGM calculada pelo método do TRS 457 para cada espessura de mama comprimida estudada na projeção MLO nos equipamentos Siemens e GE DS - MoRh para mulheres com idade inferior a 50 anos ..... 
Figura 92 Comparação entre as estimativas de DGM calculada pelo método do TRS 457 para cada espessura de mama comprimida estudada na projeção MLO nos equipamentos Siemens e GE DS - MoRh para mulheres com idade igual ou superior a 50 anos ......

Figura 93 Comparação entre as estimativas de DGM calculada pelo método de Dance para cada espessura de mama comprimida estudada na projeção $C C$ nos equipamentos Siemens e GE $700 \mathrm{~T}$ - MoMo para mulheres com idade inferior a 50 anos

Figura 94 Comparação entre as estimativas de DGM calculada pelo método de Dance para cada espessura de mama comprimida estudada na projeção CC nos equipamentos Siemens e GE 700 T - MoMo para mulheres com idade igual ou superior a 50 anos .....

Figura 95 Comparação entre as estimativas de DGM calculada pelo método de Dance para cada espessura de mama comprimida estudada na projeção MLO nos equipamentos Siemens e GE 700 T - MoMo para mulheres com idade inferior a 50 anos

Figura 96 Comparação entre as estimativas de DGM calculada pelo método de Dance para cada espessura de mama comprimida estudada na projeção MLO nos equipamentos Siemens e GE 700 T - MoMo para mulheres com idade igual ou superior a 50 anos.

Figura 97 Comparação entre as estimativas de DGM calculada pelo método de Wu para cada espessura de mama comprimida estudada na projeção CC nos equipamentos Siemens e GE 700 T - MoMo

Figura 98 Comparação entre as estimativas de DGM calculada pelo método de Wu para cada espessura de mama comprimida estudada na projeção MLO nos equipamentos Siemens e GE 700 T - MoMo

Figura 99 Comparação entre as estimativas de DGM calculada pelo método do TRS 457 para cada espessura de mama comprimida estudada na projeção CC nos equipamentos Siemens e GE 700 T - MoMo para mulheres com idade inferior a 50 anos

Figura 100 Comparação entre as estimativas de DGM calculada pelo método do TRS 457 para cada espessura de mama comprimida estudada na projeção CC nos equipamentos Siemens e GE 700 T - MoMo para mulheres com idade igual ou superior a 50 anos ....

Figura 101 Comparação entre as estimativas de DGM calculada pelo método do TRS 457 para cada espessura de mama comprimida estudada na projeção MLO nos equipamentos Siemens e GE $700 \mathrm{~T}$ - MoMo para mulheres com idade inferior a 50 anos

Figura 102 Comparação entre as estimativas de DGM calculada pelo método do TRS 457 para cada espessura de mama comprimida estudada na projeção MLO nos equipamentos Siemens e GE 700 T - MoMo para mulheres com idade igual ou superior a 50 anos 


\section{INTRODUÇÃO}

De acordo com a estimativa do Instituto Nacional do Câncer (INCA) realizado para o ano de $2008^{1}$, a mama é a segunda parte do corpo humano feminino com maior índice de incidência de câncer. Fazendo uma comparação entre os anos de $2005^{2}$ e $2008^{1}$, como mostra a Figura 1 , observa-se que, mesmo com o crescimento de aquisição de equipamentos de mamografia, houve uma variação quase imperceptível no número de casos de mulheres com câncer de mama.



Figura 1 Evolução dos tipos de câncer mais incidentes no sexo feminino de 2005 para 2008.

Em virtude deste resultado, ou seja, esta grande incidência de câncer de mama nas mulheres estar contínua, o Ministério da Saúde vem promovendo algumas campanhas em favor da realização do exame de mamografia, pelo menos a cada dois anos, para mulheres de 50 a 69 anos para a detecção precoce do câncer de mama. O Estado de São Paulo promoveu, de 2005 a 2009, mutirões de mamografia com o objetivo de detectar o câncer de mama precocemente, porém não há preocupação com a realização do controle de qualidade nestes mamógrafos. 
A mamografia é até hoje o método mais eficiente para a detecção de alguma anomalia na mama da paciente ${ }^{3}$. Porém, se o mamógrafo não for submetido a testes de controle de qualidade periódicos para se garantir a melhor qualidade de imagem junto à menor dose possível, o índice de câncer de mama pode até aumentar ${ }^{4}$.

O equipamento de mamografia necessita de um controle de qualidade rigoroso, já que é necessário e de extrema importância visualizar pequenos detalhes na radiografia para efetuar o melhor diagnóstico da paciente.

No Brasil, desde 1998, com a publicação da Portaria MS 453/98 ${ }^{5}$, tornou-se obrigatória a implementação de Programas de Garantia de Qualidade em departamentos de radiologia diagnóstica. Porém, este documento restringe-se aos cuidados com proteção radiológica e com os equipamentos emissores de radiação $X$, não adotando critérios mais detalhados para a melhoria da qualidade das imagens, após o bom funcionamento elétrico e radiológico ter sido atingido por estes equipamentos.

Portanto, torna-se fundamental a implementação dos critérios de qualidade para se obter a melhor qualidade da imagem acompanhado da menor dose à paciente. Uma publicação da European Commission ${ }^{6}$ fornece um guia completo de critérios de qualidade para imagens de diversas partes anatômicas ${ }^{7}$, inclusive a mama e em várias projeções, quantificando a qualidade obtida na imagem.

Para o estudo das doses glandular média (DGM) e na entrada da pele (DEP), deve-se seguir o princípio que a dose recebida pela paciente deve ser tão pequena quanto razoavelmente exequível (ALARA). Assim, como parte da otimização de procedimentos, primeiramente, deveria ser feito um estudo para determinar a dose à qual cada paciente está exposta durante o exame de mamografia, em seguida, avaliar a qualidade da imagem e por fim, otimizar esta dose em função da qualidade.

A relação entre dose e qualidade de imagem deve ser tal que o médico possa visualizar na imagem tudo o que pretende com a menor dose possível. Neste caso, satisfaz-se o princípio ALARA de proteção radiológica. Esta imagem será denominada de melhor qualidade da imagem, ainda que isto não seja realmente a melhor imagem no sentido real da expressão.

De acordo com o estudo realizado por Furquim ${ }^{8}$ em 2005, 66 \% dos mamógrafos do Estado de São Paulo não apresentam combinação anodo-filtro 
destinada a mamas grandes ou densas, aumentando, assim a dose média na paciente. Esse mesmo estudo também constatou um pequeno índice de contraste do filme processado, visto que a maioria dos departamentos de mamografia não possui processadora dedicada.

Por isso, é fundamental a implementação dos critérios de qualidade de imagem e otimização das doses nos departamentos de mamografia. 


\section{FINALIDADE E OBJETIVOS}

\subsection{FINALIDADE}

Encontrar a relação ALARA entre a dose e a melhor qualidade da imagem em diferentes mamógrafos

\subsection{OBJETIVOS}

- Implementar os critérios de qualidade de imagem do guia European Guidelines on Quality Criteria for Diagnostic Radiographic Images ${ }^{6}$ em mamógrafos com diferentes sistemas de aquisição de imagem em um mesmo departamento de radiologia;

- Estimar dose na entrada da pele e dose glandular média por diferentes métodos para pacientes assintomáticas;

- Desenvolver um manequim para simular a mama e estimar a dose na entrada da pele e a dose glandular média por métodos que o utilizam para medir 0 querma no ar;

- Comparar os resultados da implementação dos critérios e estudo das doses entre os mamógrafos;

- Associar a melhor qualidade da imagem à menor dose possível à paciente. 


\section{FUNDAMENTOS TEÓRICOS}

\subsection{FUNCIONAMENTO DOS MAMÓGRAFOS}

A Figura 2 mostra um esquema de um mamógrafo. $O$ equipamento de mamografia é composto por:

Gerador de raios X (fonte de alta tensão);

Controle automático de exposição

Tubo de raios $X$;

Compressor da mama;

Pedal de controle;

Grades;

Mesa;

Barreira de proteção;

Colimadores;

Cúpula;

Painel de controle;

Sistema ergonômico;

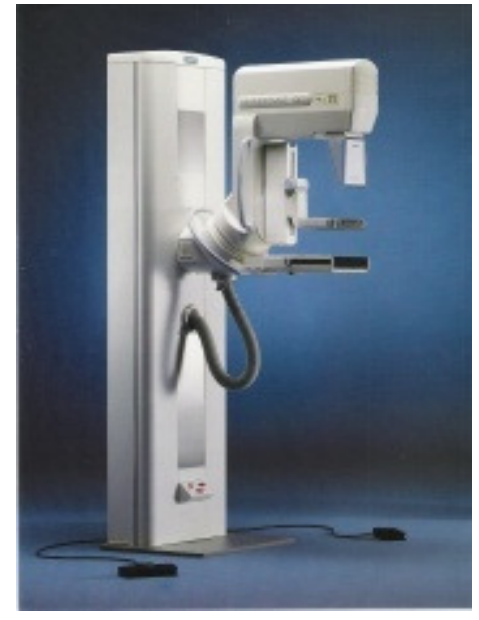

Figura 2 Equipamento de mamografia. (Folheto de propaganda da Siemens)

Sistema de detecção de imagem;

O gerador de raios $\mathbf{X}$ fornece energia elétrica para o tubo de raios $X$ permite a seleção do produto corrente-tempo e da tensão de aceleração dos elétrons ${ }^{9}$. Esses parâmetros são selecionados pelo controle automático de exposição. Geralmente, o tipo de gerador utilizado em mamografia é o de alta frequência por apresentar mínima variação de voltagem (2 \%) e uma resposta rápida e reprodutível.

O tubo de raios $\mathbf{X}$ fica contido em uma ampola a vácuo ${ }^{9}$, é composto por um anodo e um catodo e possui uma geometria um pouco diferente dos tubos utilizados nos equipamentos convencionais, como mostra a Figura 3. O anodo giratório pode ser constituído por molibdênio (Mo), ródio (Rh) ou tungstênio (W). Os filtros utilizados no equipamento de mamografia têm o objetivo de atenuar seletivamente e de otimizar o espectro do feixe de raios $\mathrm{X}$. As combinações anodo-filtro mais frequentes nos mamógrafos são Mo/Mo, W/Rh, W/Mo, Mo/Rh e $\mathrm{Rh} / \mathrm{Rh}$. 


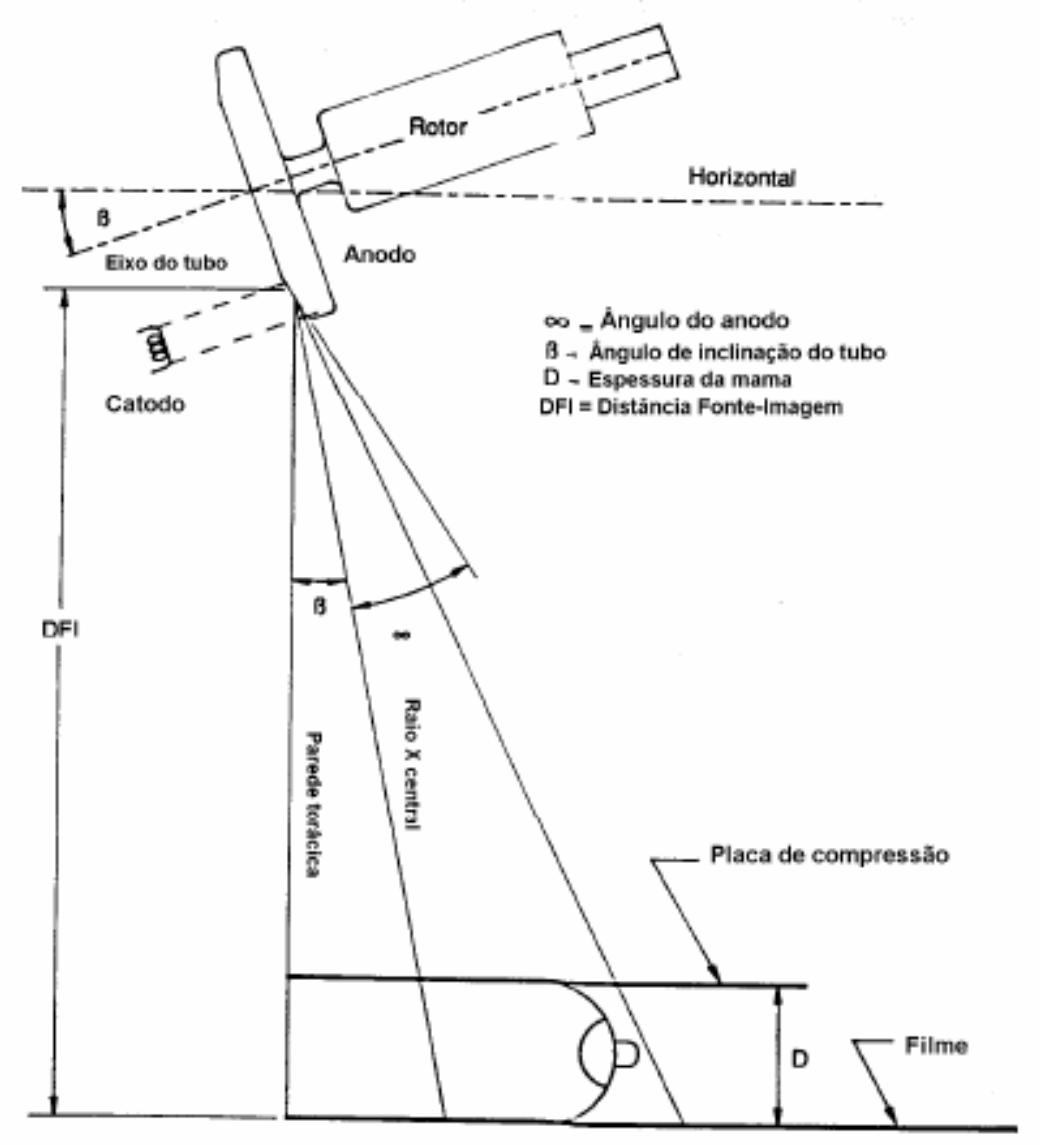

Figura 3 Geometria do tubo de raios X mamográfico ${ }^{10}$.

Os espectros de raios $X$ ilustrados na Figura 4 mostram que a combinação Mo/Mo é mais indicada para tamanhos de mama menores e menos densas, visto que os raios $X$ do espectro são emitidos em energias mais baixas.

As combinações $\mathrm{W} / \mathrm{Rh}, \mathrm{Mo} / \mathrm{Rh}$ e $\mathrm{Rh} / \mathrm{Rh}$ emitem raios $\mathrm{X}$ em energias maiores, portanto são indicados para tamanho de mamas maiores e mais densas ${ }^{11}$.

O compressor da mama tem uma importância fundamental na qualidade da imagem mamográfica, pois faz com que a espessura da mama torne-se mais homogênea a fim de obter uma imagem com boa qualidade, uma exposição melhor distribuída e reduzir as estruturas sobrepostas. Além disso, o compressor minimiza o movimento da paciente, evitando borramento, reduz a dose em virtude da redução da espessura e afasta a mama da parede torácica, fazendo com que a imagem mamográfica contenha apenas as estruturas da mama, não apresentando estruturas ósseas. 

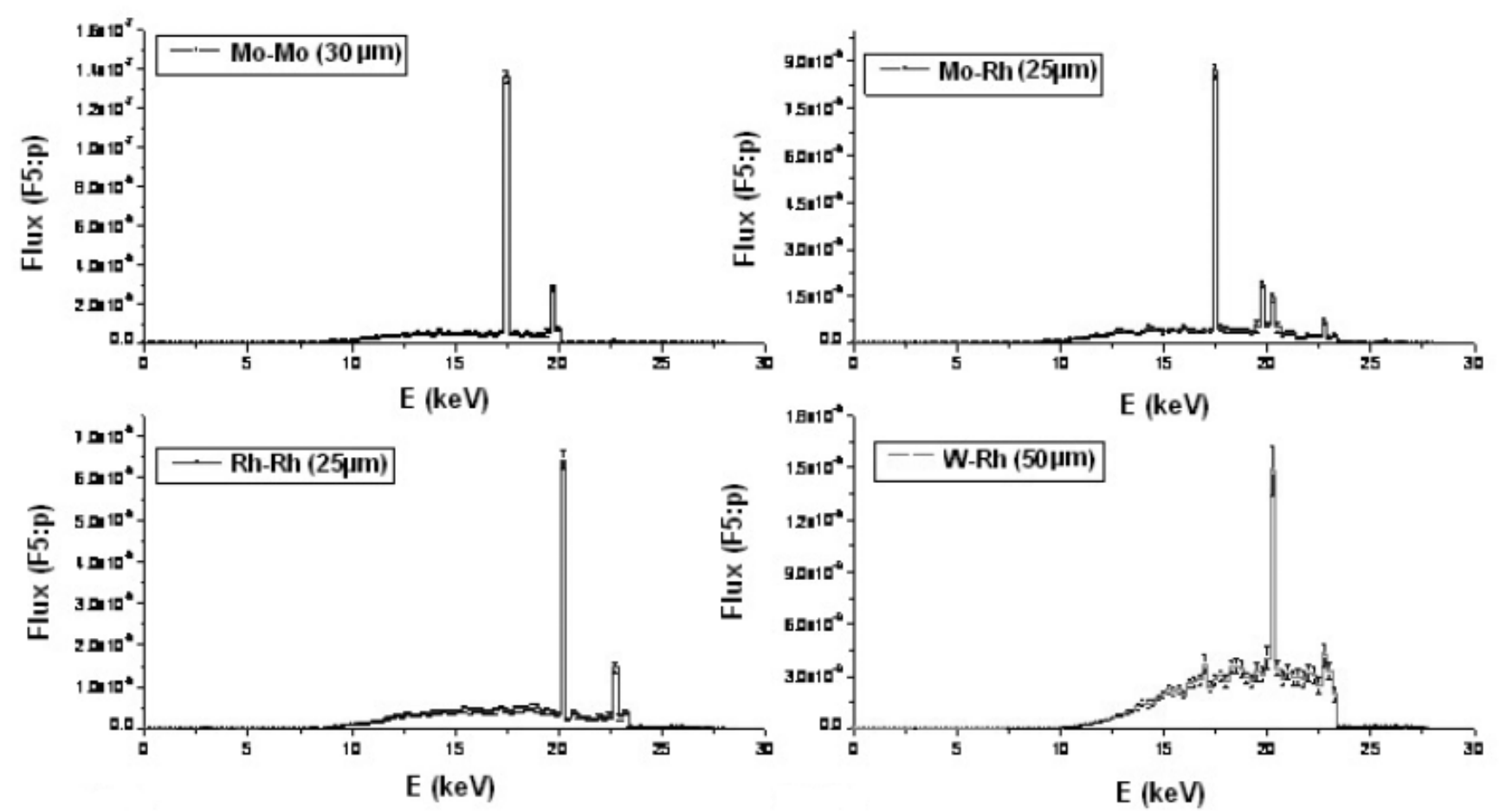

Figura 4 Espectro de energia dos raios $X$ para as combinações anodo-filtro (a) Mo/Mo, (b) Mo/Rh, (c) Rh/Rh e (d) W/Rh' ${ }^{12}$.

O pedal de controle tem a função de movimentar o compressor da mama para cima ou para baixo.

A grade é composta por tiras de chumbo $(\mathrm{Pb})$, servem para reduzir o espalhamento da radiação, evitando o borramento da imagem.

A barreira de proteção tem a função apenas de impedir que a paciente exponha à radiação alguma parte do corpo, além da mama.

As funções em comum da mesa dos equipamentos convencionais e digitais é servir de suporte da mama e é onde se localiza a grade do equipamento. No equipamento convencional, o chassi é posicionado na mesa. Já no digital, o sistema de detecção da imagem encontra-se instalado neste dispositivo.

Os colimadores têm a função de colimar o feixe de radiação para que este atinja somente a região de interesse, ou seja, a mama.

A cúpula contém o tubo de raios $X$, os colimadores e o filtro.

O painel de controle localiza-se geralmente atrás de um biombo de vidro plumbífero, permitindo à técnica (operador) visualizar a paciente durante a realização do exame. 
O sistema que une a cúpula ao sistema de aquisição da imagem é ergonômico. Ele tem um formato de um arco-em-C para facilitar o posicionamento da paciente no equipamento mamográfico e pode ser girado em um ângulo de até $180^{\circ}$ em relação à posição vertical para aquisição de imagens laterais da mama.

O sistema de detecção de imagem de um mamógrafo difere em equipamentos que contêm o sistema écran-filme e o sistema digital. No sistema écran-filme (convencional) os fótons de raios $X$, que atravessam a mama, interagem com o filme mamográfico que contém emulsão em um único lado. Esse filme será posteriormente processado em um sistema contendo revelador, fixador, água e um sistema de secagem ${ }^{9}$. Para obter uma boa qualidade da imagem é necessário realizar um controle de alguns parâmetros da processadora, como temperatura e velocidade de processamento do filme. Esta é a grande diferença entre o sistema écran-filme e o digital. O sistema digital dispensa o uso da processadora, portanto não há filme e o sistema de detecção de imagem é um tanto quanto diferente. Existem dois tipos de sistemas digitais ${ }^{8}$ :

- Indireto: Os raios X são convertidos em sinal de luz e, em seguida, em cargas elétricas. Ex: Csl:TI - aSi e Csl:TI - CCD

- Direto: Os raios X são convertidos diretamente em cargas elétricas. Ex: selênio amorfo (a-Se). 


\subsection{CONTROLE DE QUALIDADE NOS MAMÓGRAFOS}

Para um departamento de radiologia diagnóstica garantir a qualidade do seu serviço é imprescindível a implementação de um programa de controle de qualidade nos seus equipamentos.

Os testes de reprodutibilidade e repetibilidade da tensão de aceleração de elétrons $(\mathrm{kV})$ têm o objetivo de garantir que o valor de tensão selecionado ou indicado no painel de controle seja próximo ao que realmente é utilizado durante o exame e verificar se é reprodutível.

O tempo de exposição também é avaliado, pois não deve ser longo, para evitar o movimento da paciente e não causar o enegrecimento excessivo do filme.

Para avaliar a qualidade do feixe de raios $X$ é preciso saber o valor da camada semi-redutora (CSR), que é a espessura necessária de um material absorvedor para reduzir pela metade a intensidade do feixe de radiação e pode ser calculada por $^{13}$ :

$$
\operatorname{CSR}=\frac{x_{b} \cdot\left(\ln .2 \frac{L_{a}}{L_{0}}\right)-x_{a} \cdot\left(\ln 2 \frac{L_{b}}{L_{0}}\right)}{\left(\ln \frac{L_{a}}{L_{b}}\right)}
$$

onde:

$L_{0}=$ leitura inicial de exposição.

$L_{a}=$ leitura de exposição imediatamente superior a $L_{0} / 2$.

$L_{b}=$ leitura de exposição imediatamente inferior a $L_{0} / 2$.

$x_{a}=$ espessura de Al correspondente à leitura $L_{a}$.

$x_{b}=$ espessura de Al correspondente à leitura $L_{b}$.

O valor da CSR é diferente para cada tensão de aceleração de elétrons e combinação anodo-filtro, pois quanto maior a tensão, maior será a espessura de alumínio necessária para reduzir o feixe à metade.

Por meio de medições do querma no ar determina-se o rendimento do tubo de raios $\mathbf{X}$ para saber se a taxa de querma no ar medida para certa tensão de aceleração de elétrons está adequada para uma boa qualidade da imagem. 
O teste de linearidade e reprodutibilidade do querma no ar também tem a sua importância no controle de qualidade, pois é utilizado para verificar se à medida que se aumenta o valor do produto corrente-tempo, mantendo a tensão constante, o querma no ar aumenta linearmente e se é reprodutível ${ }^{8}$.

A qualidade da imagem é avaliada por meio da visualização de estruturas na imagem mamográfica de manequins.

Para evitar um dano na paciente ou uma má qualidade de imagem, o sistema de compressão da mama deve ser avaliado para que a força de compressão não seja nem grande e nem pequena.

O teste de colimação é realizado para que nenhuma região do corpo da paciente seja irradiada desnecessariamente e também para garantir que a região da mama iluminada apareça realmente na imagem.

A imagem mamográfica deve possibilitar a visualização de detalhes para que o radiologista realize o diagnóstico correto da paciente. Esta característica é garantida por meio da avaliação da resolução do equipamento.

O tamanho do ponto focal é avaliado, pois a resolução depende diretamente deste fator.

A luminância do negatoscópio é avaliada para garantir que todas as estruturas presentes no filme mamográfico possam ser visualizadas pelo radiologista.

É necessário realizar o teste da compensação e reprodutibilidade do controle automático de exposição, pois a densidade ótica do filme não pode sofrer grandes variações mesmo modificando-se a espessura ou a densidade da mama da paciente e o sistema deve ser reprodutível.

A verificação do movimento da grade anti-espalhamento é realizada para evitar o aparecimento da grade na imagem mamográfica. Além desta verificação também se analisa se há artefatos na imagem para que o radiologista não efetue um diagnóstico incorreto.

Em sistemas com filmes, para avaliar as condições da processadora, são realizados alguns testes. Dentre eles encontram-se a determinação do índice de contraste do filme processado e a sensitometria diária.

O controle automático de densidades também é avaliado já que ele tem a função de ser um ajuste fino para uma boa qualidade da imagem. 
Os detectores do sistema digital devem ser avaliados para que todos forneçam um sinal real para a formação de uma boa imagem. Esse parâmetro é avaliado testando-se a uniformidade do campo horizontal.

A medição da função de transferência de modulação proporciona a descrição mais completa da resolução espacial de um detector. A resolução espacial, por sua vez, deve permitir a visualização de pequenos objetos em alto contraste $^{14}$.

A razão contraste-ruído é determinada e espera-se obter o máximo valor de contraste junto ao mínimo de ruído, evitando-se que a dose ultrapasse os níveis de referência.

\subsubsection{DOSE NA ENTRADA DA PELE}

Não é possível determinar o valor exato da dose na entrada da pele (DEP), mas é importante controlar este parâmetro para que o valor desta dose não ultrapasse os níveis de referência estabelecidos, preservando assim a saúde da paciente. Estes níveis de referência serão apresentados adiante, quando forem necessários e o valor da DEP será estimado por diferentes métodos.

\subsubsection{MÉTODO DO GUIA DA ANVISA}

O Guia "Radiodiagnóstico Médico: Segurança e Desempenho de Equipamentos" da Agência Nacional de Vigilância Sanitária (ANVISA) ${ }^{13}$ foi elaborado para descrever os procedimentos para a realização de alguns testes de controle de qualidade para equipamentos de radiodiagnóstico médico.

Dentre estes testes, inclui-se a estimativa da dose na entrada da pele (DEP), que é determinada pela equação 2 .

$$
D=K_{a r} \cdot k_{P T} \cdot f_{c} \cdot F R E
$$

onde:

$K_{a r}=$ querma no ar.

$k_{P T}=$ fator de correção para temperatura e pressão.

$f_{c}=$ fator de calibração da câmara.

$F R E=$ fator de retro-espalhamento . 
O querma é medido apenas com a câmara de ionização no centro do feixe, ou seja, sem manequim. Por isso, há uma correção no valor do querma para simular o retro-espalhamento da radiação e foram adotados os fatores determinados por Kramer e colaboradores ${ }^{15}$, que se encontram na Tabela 1.

Tabela 1 Fatores de retro-espalhamento para diferentes valores de camada semiredutora determinados por Kramer e colaboradores ${ }^{15}$.

\begin{tabular}{|c|c|c|c|c|c|c|c|c|c|}
\hline CSR (mmAl) & 0,25 & 0,30 & 0,35 & 0,40 & 0,45 & 0,50 & 0,55 & 0,60 & 0,65 \\
\hline FRE & 1,07 & 1,07 & 1,08 & 1,09 & 1,10 & 1,11 & 1,12 & 1,12 & 1,13 \\
\hline
\end{tabular}

O nível de referência determinado por este método para uma mama padrão $(4,5 \mathrm{~cm})$ é $10 \mathrm{mGy}$.

\subsubsection{MÉTODO DO GUIA DO ACR}

O "Mammography Quality Control Manual" elaborado pela American College of Radiology $(\mathrm{ACR})^{16}$ contempla os testes que devem ser realizados em um controle de qualidade em mamografia. Dentre eles, encontra-se a estimativa da dose na entrada da pele que é determinada por meio da equação 3.

$$
D=K_{a r} \cdot k_{P T} \cdot f_{c}
$$

Esta equação difere da anterior (equação 2) apenas por não apresentar o fator de retro-espalhamento (FRE). Isto ocorre uma vez que a medição do querma é realizada com o manequim ao lado da câmara de ionização.

Este método não apresenta nível de referência.

\subsubsection{DOSE GLANDULAR MÉDIA}

A dose glandular média (DGM) é a média da dose recebida pela região glandular da mama. Não é possível medir diretamente a DGM à qual a paciente está exposta durante um exame de mamografia. Porém, como o tecido glandular é a região mais radiosensível da mama, é de suma importância estimar esta dose $^{3}$. Para isto, foram adotadas algumas metodologias desenvolvidas por pesquisadores. 


\subsubsection{MÉTODO DE DANCE}

O estudo inicial para estimativa de dose glandular foi realizado apenas por Dance $^{17}$ em 1990. Por meio do Cálculo de Monte Carlo foram determinados fatores de conversão que aproximassem o querma medido da dose glandular média.

$$
D=K \cdot g
$$

onde:

$K=$ querma no ar incidente;

$g=$ fator de conversão de querma no ar em dose glandular média para mama com $50 \%$ de glandularidade.

Em 2000, um grupo de estudiosos europeus, incluindo Dance ${ }^{18}$, publicaram o estudo para a determinação de mais fatores de conversão para a estimativa da dose glandular média. Portanto, a dose passou a ser estimada por meio da equação 5 .

$$
D=K . g . c . s
$$

onde:

$K=$ querma no ar incidente na superfície da mama, medido sem retroespalhamento;

$g$ = fator de conversão do querma incidente na mama, em dose glandular média, para glandularidade de $50 \%$;

$c=$ fator de correção para diferentes composições da mama com glandularidade diferente de $50 \%$;

$s=$ fator de correção para diferentes combinações anodo-filtro.

A glandularidade da mama de uma paciente pode ser estimada aplicando-se $o$ seguinte polinômio:

$$
\text { glandularidade }(\%)=a t^{3}+b t^{2}+c t+d
$$

Os coeficientes $a, b, c$ e d estão definidos na Tabela 2 e o parâmetro $t$ representa a espessura da mama comprimida da paciente. 
Tabela 2 Coeficientes para o ajuste polinomial da glandularidade em função da espessura da mama ${ }^{18}$.

\begin{tabular}{ccc}
\hline Coeficiente & Idade entre 40 e 49 anos & Idade entre 50 e 64 anos \\
\hline A & 0,00005209 & $-0,0001118$ \\
B & 0,00125494 & 0,03932 \\
C & $-1,988$ & $-4,544$ \\
D & 138,8 & 176,0 \\
\hline
\end{tabular}

Esta publicação de Dance e colaboradores ${ }^{18}$ fornece os fatores de correção $\mathrm{g}$ (Tabela 3), s (Tabela 4) e c (Tabela 5) para algumas espessuras de mama, para algumas CSR e para algumas glandularidades da mama. Para obter os valores desses fatores para todas as espessuras de mama desejadas, é necessário realizar interpolações lineares.

Tabela 3 Fatores de conversão g (mGy/mGy) para espessuras de mama de 2 a $11 \mathrm{~cm}$ e CSR entre 0,30 a $0,60 \mathrm{mmAl}^{18}$.

\begin{tabular}{cccccccc}
\hline $\begin{array}{c}\text { Espessura } \\
\text { (cm) }\end{array}$ & 0,3 & 0,35 & 0,4 & 0,45 & 0,5 & 0,55 & 0,6 \\
\hline 2 & 0,39 & 0,433 & 0,473 & 0,509 & 0,543 & 0,573 & 0,587 \\
3 & 0,274 & 0,309 & 0,342 & 0,374 & 0,406 & 0,437 & 0,466 \\
4 & 0,207 & 0,235 & 0,261 & 0,289 & 0,318 & 0,346 & 0,374 \\
4,5 & 0,183 & 0,208 & 0,232 & 0,258 & 0,285 & 0,311 & 0,339 \\
5 & 0,164 & 0,187 & 0,209 & 0,232 & 0,258 & 0,287 & 0,31 \\
6 & 0,135 & 0,154 & 0,172 & 0,192 & 0,214 & 0,236 & 0,261 \\
7 & 0,114 & 0,13 & 0,145 & 0,163 & 0,177 & 0,202 & 0,224 \\
8 & 0,098 & 0,112 & 0,126 & 0,14 & 0,154 & 0,175 & 0,195 \\
9 & 0,0859 & 0,0981 & 0,1106 & 0,1233 & 0,1357 & 0,1543 & 0,1723 \\
10 & 0,0763 & 0,0873 & 0,0986 & 0,1096 & 0,1207 & 0,1375 & 0,154 \\
11 & 0,0687 & 0,0786 & 0,0887 & 0,0988 & 0,1088 & 0,124 & 0,1385 \\
\hline
\end{tabular}

Tabela 4 Fatores de conversão $s$ do espectro utilizados clinicamente e erros máximos que podem ocorrer quando são utilizados ${ }^{18}$.

\begin{tabular}{|c|c|c|}
\hline Espectro & fator s & Erro máx (\%) \\
\hline $\mathrm{Mo} / \mathrm{Mo}$ & 1 & 3,1 \\
\hline $\mathrm{Mo} / \mathrm{Rh}$ & 1,017 & 2,2 \\
\hline $\mathrm{Rh} / \mathrm{Rh}$ & 1,061 & 3,6 \\
\hline $\mathrm{Rh} / \mathrm{Al}$ & 1,044 & 2,4 \\
\hline $\mathrm{W} / \mathrm{Rh}$ & 1,042 & 2,1 \\
\hline
\end{tabular}


Tabela 5 Fatores de conversão c para glandularidades de 0,1 a $100 \%$ na região central da mama, espessuras de mama de 2 a $11 \mathrm{~cm}$ e CSR de 0,30 a 0,60 $\mathrm{mmAl}^{18}$.

\begin{tabular}{|c|c|c|c|c|c|c|c|c|c|c|c|c|c|}
\hline \multirow{2}{*}{$\begin{array}{c}\text { HVL } \\
(\mathrm{mmAl})\end{array}$} & \multirow{2}{*}{$\begin{array}{c}\text { Espessura } \\
\text { (cm) }\end{array}$} & \multicolumn{5}{|c|}{ Glanduralidade da mama } & \multirow[t]{2}{*}{ HVL } & \multirow{2}{*}{$\begin{array}{c}\text { Espessura } \\
\text { (cm) }\end{array}$} & \multicolumn{5}{|c|}{ Glanduralidade da mama } \\
\hline & & $0,10 \%$ & $25 \%$ & $50 \%$ & $75 \%$ & $100 \%$ & & & $0,10 \%$ & $25 \%$ & $50 \%$ & $75 \%$ & $100 \%$ \\
\hline 0,3 & 2 & 1,13 & 1,059 & 1 & 0,938 & 0,885 & 0,35 & 2 & 1,123 & 1,058 & 1 & 0,943 & 0,891 \\
\hline 0,3 & 3 & 1,206 & 1,098 & 1 & 0,915 & 0,836 & 0,35 & 3 & 1,196 & 1,09 & 1 & 0,919 & 0,842 \\
\hline 0,3 & 4 & 1,253 & 1,12 & 1 & 0,898 & 0,808 & 0,35 & 4 & 1,244 & 1,112 & 1 & 0,903 & 0,816 \\
\hline 0,3 & 5 & 1,282 & 1,127 & 1 & 0,886 & 0,794 & 0,35 & 5 & 1,272 & 1,121 & 1 & 0,89 & 0,801 \\
\hline 0,3 & 6 & 1,303 & 1,135 & 1 & 0,882 & 0,785 & 0,35 & 6 & 1,294 & 1,132 & 1 & 0,886 & 0,793 \\
\hline 0,3 & 7 & 1,317 & 1,142 & 1 & 0,881 & 0,784 & 0,35 & 7 & 1,308 & 1,138 & 1 & 0,886 & 0,788 \\
\hline 0,3 & 8 & 1,325 & 1,143 & 1 & 0,879 & 0,78 & 0,35 & 8 & 1,312 & 1,14 & 1 & 0,884 & 0,786 \\
\hline 0,3 & 9 & 1,328 & 1,145 & 1 & 0,879 & 0,78 & 0,35 & 9 & 1,319 & 1,145 & 1 & 0,884 & 0,786 \\
\hline 0,3 & 10 & 1,329 & 1,147 & 1 & 0,88 & 0,78 & 0,35 & 10 & 1,319 & 1,144 & 1 & 0,881 & 0,785 \\
\hline 0,3 & 11 & 1,328 & 1,143 & 1 & 0,879 & 0,779 & 0,35 & 11 & 1,322 & 1,142 & 1 & 0,882 & 0,784 \\
\hline 0,4 & 2 & 1,111 & 1,054 & 1 & 0,949 & 0,9 & 0,45 & 2 & 1,099 & 1,052 & 1 & 0,948 & 0,905 \\
\hline 0,4 & 3 & 1,181 & 1,087 & 1 & 0,922 & 0,851 & 0,45 & 3 & 1,169 & 1,08 & 1 & 0,924 & 0,858 \\
\hline 0,4 & 4 & 1,227 & 1,105 & 1 & 0,907 & 0,825 & 0,45 & 4 & 1,209 & 1,102 & 1 & 0,909 & 0,829 \\
\hline 0,4 & 5 & 1,258 & 1,12 & 1 & 0,899 & 0,81 & 0,45 & 5 & 1,248 & 1,115 & 1 & 0,898 & 0,815 \\
\hline 0,4 & 6 & 1,276 & 1,125 & 1 & 0,89 & 0,798 & 0,45 & 6 & 1,267 & 1,125 & 1 & 0,891 & 0,801 \\
\hline 0,4 & 7 & 1,292 & 1,132 & 1 & 0,887 & 0,793 & 0,45 & 7 & 1,283 & 1,129 & 1 & 0,892 & 0,797 \\
\hline 0,4 & 8 & 1,302 & 1,136 & 1 & 0,885 & 0,79 & 0,45 & 8 & 1,298 & 1,137 & 1 & 0,887 & 0,799 \\
\hline 0,4 & 9 & 1,308 & 1,138 & 1 & 0,884 & 0,789 & 0,45 & 9 & 1,301 & 1,135 & 1 & 0,886 & 0,792 \\
\hline 0,4 & 10 & 1,311 & 1,138 & 1 & 0,883 & 0,788 & 0,45 & 10 & 1,305 & 1,138 & 1 & 0,886 & 0,791 \\
\hline 0,4 & 11 & 1,315 & 1,14 & 1 & 0,885 & 0,791 & 0,45 & 11 & 1,312 & 1,138 & 1 & 0,885 & 0,789 \\
\hline 0,5 & 2 & 1,098 & 1,05 & 1 & 0,955 & 0,91 & 0,55 & 2 & 1,086 & 1,043 & 1 & 0,955 & 0,914 \\
\hline 0,5 & 3 & 1,164 & 1,078 & 1 & 0,928 & 0,864 & 0,55 & 3 & 1,154 & 1,071 & 1 & 0,932 & 0,87 \\
\hline 0,5 & 4 & 1,209 & 1,094 & 1 & 0,912 & 0,835 & 0,55 & 4 & 1,196 & 1,093 & 1 & 0,918 & 0,843 \\
\hline 0,5 & 5 & 1,242 & 1,111 & 1 & 0,903 & 0,817 & 0,55 & 5 & 1,227 & 1,105 & 1 & 0,906 & 0,824 \\
\hline 0,5 & 6 & 1,263 & 1,12 & 1 & 0,896 & 0,807 & 0,55 & 6 & 1,252 & 1,115 & 1 & 0,9 & 0,814 \\
\hline 0,5 & 7 & 1,278 & 1,127 & 1 & 0,89 & 0,8 & 0,55 & 7 & 1,267 & 1,122 & 1 & 0,896 & 0,805 \\
\hline 0,5 & 8 & 1,289 & 1,132 & 1 & 0,889 & 0,794 & 0,55 & 8 & 1,278 & 1,125 & 1 & 0,89 & 0,8 \\
\hline 0,5 & 9 & 1,295 & 1,134 & 1 & 0,887 & 0,793 & 0,55 & 9 & 1,285 & 1,128 & 1 & 0,89 & 0,798 \\
\hline 0,5 & 10 & 1,302 & 1,138 & 1 & 0,886 & 0,791 & 0,55 & 10 & 1,29 & 1,133 & 1 & 0,889 & 0,796 \\
\hline 0,5 & 11 & 1,303 & 1,14 & 1 & 0,885 & 0,789 & 0,55 & 11 & 1,293 & 1,134 & 1 & 0,888 & 0,793 \\
\hline 0,6 & 2 & 1,089 & 1,045 & 1 & 0,959 & 0,919 & & & & & & & \\
\hline 0,6 & 3 & 1,142 & 1,065 & 1 & 0,933 & 0,874 & & & & & & & \\
\hline 0,6 & 4 & 1,185 & 1,09 & 1 & 0,923 & 0,85 & & & & & & & \\
\hline 0,6 & 5 & 1,216 & 1,102 & 1 & 0,91 & 0,83 & & & & & & & \\
\hline 0,6 & 6 & 1,238 & 1,113 & 1 & 0,904 & 0,82 & & & & & & & \\
\hline 0,6 & 7 & 1,252 & 1,12 & 1 & 0,899 & 0,812 & & & & & & & \\
\hline 0,6 & 8 & 1,266 & 1,123 & 1 & 0,894 & 0,806 & & & & & & & \\
\hline 0,6 & 9 & 1,272 & 1,124 & 1 & 0,893 & 0,801 & & & & & & & \\
\hline 0,6 & 10 & 1,279 & 1,125 & 1 & 0,891 & 0,797 & & & & & & & \\
\hline 0,6 & 11 & 1,284 & 1,129 & 1 & 0,893 & 0,798 & & & & & & & \\
\hline
\end{tabular}




\subsubsection{MÉTODO DE WU}

Paralelamente à publicação de Dance ${ }^{18}$, um grupo de estudiosos americanos, incluindo $\mathrm{Wu}^{19}$, mostraram que a dose glandular deve ser estimada pela equação 7, baseada em medições do querma realizadas com o manequim ao lado da câmara de ionização.

$$
D_{g}=D_{g N} \cdot X_{e s e}
$$

onde:

$D_{g}=$ dose glandular média;

$D_{g N}=$ dose glandular média normalizada;

$X_{\text {ese }}=$ exposição de entrada na pele

Wu e colaboradores ${ }^{20}$ apresentaram uma tabela em trabalho publicado em 1991 onde constavam valores de $D_{g N}$ em função da CSR, tensão de aceleração de elétrons, espessura e composição da mama para a combinação anodo-filtro Mo/Mo.

Em 1997, Sobol e $\mathrm{Wu}^{21}$ apresentaram um algoritmo que facilitou a determinação da $D_{g N}$ para qualquer tensão de aceleração de elétrons, CSR, espessura da mama, combinação da mama e anodo-filtro por meio de interpolações.

\subsubsection{MÉTODO DO TRS 457}

O método apresentado pelo Código de Prática TRS $457^{22}$, da International Atomic Energy Agency (IAEA), baseia-se no método de Dance ${ }^{17,18}$ ao adotar os mesmos fatores de conversão, entretanto difere ao se analisar o método de obtenção do valor do querma no ar.

Primeiramente, realiza-se as medições de querma no ar para uma mesma espessura de mama comprimida, que será a espessura de referência utilizando apenas a câmara de ionização, ou seja, neste método o manequim não é utilizado. O querma é medido para diferentes valores de tensão de aceleração de elétrons nesta mesma espessura. 
Em seguida, determina-se o rendimento do tubo de raios $X$ para cada valor de tensão escolhido. O rendimento é calculado aplicando a equação 8:

$$
Y_{r e f}=\frac{\bar{M} N_{K, Q_{0}} k_{Q} k_{T P}}{P_{I t}}
$$

onde:

$\bar{M}=$ média das leituras do dosímetro;

$N_{K, Q_{0}}=$ coeficiente de calibração da câmara;

$k_{Q}=$ fator de correção para diferenças na resposta do dosímetro na qualidade de calibração e medição;

$k_{T P}=$ fator de correção para temperatura e pressão;

$P_{I t}=$ produto corrente-tempo.

Com os valores de rendimento conhecidos, calcula-se o querma no ar para qualquer paciente por meio da equação 9.

$$
K_{i}=Y_{r e f}\left(\frac{d_{p}-d_{r e f}}{d_{p}-d_{B}}\right)^{2} P_{I t, p a t}
$$

onde:

$Y_{r e f}=$ rendimento do tubo de raios $\mathrm{X}$ na espessura de referência;

$d_{p}=$ distância entre o ponto focal e a parte superior do suporte da mama;

$d_{r e f}=$ distância entre a parte superior do suporte da mama e o ponto de referência;

$d_{B}=$ distância entre a parte superior do suporte da mama e a parte superior da mama;

$P_{I t, p a t}=$ produto corrente-tempo registrado na exposição da paciente.

Por fim, estima-se a dose glandular média aplicando a equação 10:

$$
D_{G}=c_{D_{G 50}, K_{i}} c_{D_{G_{g}}, D_{G 50}} s K_{i}
$$

onde:

$c_{D_{G 50}, K_{i}}=$ fator de conversão do querma no ar para dose glandular média para uma mama com glandularidade de $50 \%$;

$c_{D_{G g}, D_{G 50}}=$ fator de correção para mama com glandularidade diferente de $50 \%$; 
$s=$ fator de correção para as diferentes combinações de anodo-filtro;

$K_{i}=$ querma no ar.

Este método leva em consideração o rendimento do tubo, já que é este resultado que demonstra se a qualidade da imagem será suficiente para um bom diagnóstico ${ }^{8}$.

\subsection{CRITÉRIOS DE QUALIDADE DE IMAGEM}

Em um exame de mamografia rotineiro, são realizadas 4 radiografias da paciente, 2 na projeção Crânio Caudal e 2 na projeção Médio Lateral Oblíqua. Porém, existem outras projeções (Figura 5) que podem ser feitas, caso seja solicitado pelo radiologista. A projeção é definida de acordo com a necessidade do diagnóstico.

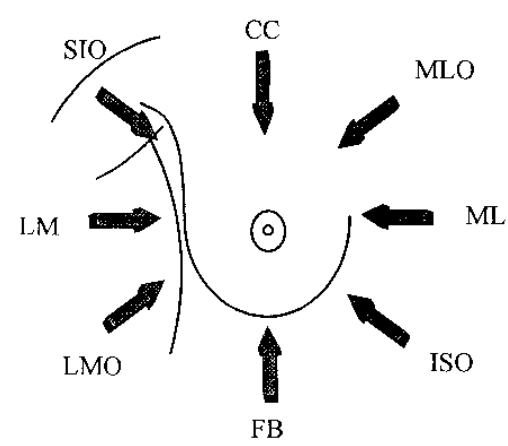

CC = Crânio Caudal

MLO = Médio Lateral Oblíqua

$M L=$ Médio Lateral $\left(90^{\circ}\right)$

ISO = Ínfero-Superior Oblíqua

FB = Crânio Caudal (de baixo)

LMO = Látero-medial Oblíqua

LM = Látero-medial $\left(90^{\circ}\right)$

SIO = Súpero-inferior Obíqua

Figura 5 Diferentes projeções da mama que podem ser obtidas em um exame de mamografia $^{23}$.

A imagem mamográfica deve apresentar alguns critérios de qualidade essenciais para a realização de um bom diagnóstico. O guia European Guidelines on Quality Criteria for Diagnostic Radiographic Images ${ }^{6}$ apresenta esses critérios para algumas estruturas do corpo, inclusive a mama. Foram estabelecidos 11 critérios para a projeção Crânio Caudal (CC) e 12 critérios para a projeção Médio Lateral Oblíqua (MLO). Estes critérios estão descritos a seguir.

- Critérios de imagem relacionados ao posicionamento da paciente:

1 músculo peitoral projetado no ângulo correto; 
2 visualização do ângulo inframamário;

3 mamilo inteiro de perfil, sem sobreposição de tecido mamário;

4 nenhuma dobra da pele visível;

5 imagens simétricas da mama direita e esquerda.

- Critérios de imagem relacionados ao desempenho, parâmetros de exposição e movimento da paciente

1 visualização do tecido glandular lateral;

2 visualização de cordões fibrosos no tecido adiposo;

3 visualização de estruturas vasculares no tecido adiposo;

4 visualização do tecido da mama medial;

5 visualização do tecido adiposo retro-glandular;

6 visualização da margem do músculo peitoral;

7 visualização do contorno brilhante da pele;

8 visualização da estrutura da pele ao longo do músculo peitoral. 


\section{EQUIPAMENTOS, MATERIAIS E METODOLOGIA}

\subsection{Equipamentos e materiais}

O estudo foi desenvolvido no Instituto de Radiologia do Hospital das Clínicas da Faculdade de Medicina da USP, na cidade de São Paulo/SP. Foram utilizados três mamógrafos ilustrados na Figura 6: GE Senographe 700 T (convencional), Siemens Mammomat 3000 Nova (convencional) e GE Senographe DS (digital).
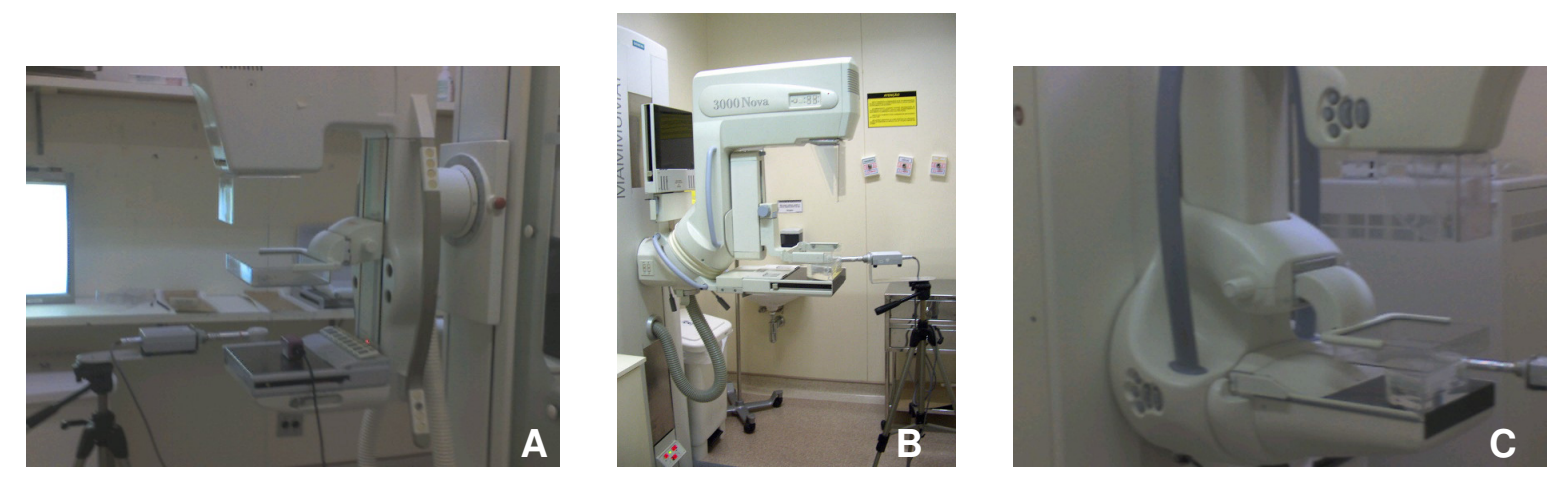

Figura 6 Equipamentos de mamografia estudados: A. GE Senographe 700T. B. Siemens Mammomat 3000 Nova. C. GE Senographe DS

A fim de obter a DGM pelo método de $\mathrm{Wu}^{19,20,21}$ e a DEP pelo método do $\mathrm{ACR}^{16}$ foi desenvolvido um manequim da mama no ex Centro de Metrologia das Radiações (CMR), atual Gerência de Metrologia das Radiações (GMR), do Instituto de Pesquisas Energéticas e Nucleares (IPEN/CNEN). Este objeto é composto por placas de PMMA, com diferentes espessuras, para simular os diversos tamanhos da mama comprimida das pacientes nas projeções Crânio Caudal (CC) e Médio Lateral Oblíqua (MLO). Foram produzidas: 1 placa de $2 \mathrm{~cm}$, 10 de $0,5 \mathrm{~cm}$ e 5 de $0,3 \mathrm{~cm}$.

Para medir o querma no ar, foi utilizado um conjunto Radcal Co. contendo uma câmara de ionização de $6 \mathrm{~cm}^{3}$ dedicada a mamografia e um monitor 9010 . Este conjunto foi utilizado para obter os valores das camadas semi-redutoras (CSR), DEP e DGM. Simultaneamente à medida de querma no ar, foi medida a tensão de aceleração de elétrons do tubo de raios $X(k V p)$ e o tempo de exposição com o medidor da Radcal Co. (modelo 4083). Estes dois parâmetros foram registrados 
para avaliar as condições de funcionamento do mamógrafo e obter a tensão real fornecida no feixe de raios $X$.

Foram utilizados filtros de alumínio 1100 (99 \% de pureza) com diferentes espessuras para a determinação das CSR. Todos os equipamentos de medição pertencem ao Instituto de Eletrotécnica e Energia da Universidade de São Paulo (IEE/USP) e podem ser vistos na Figura 7.

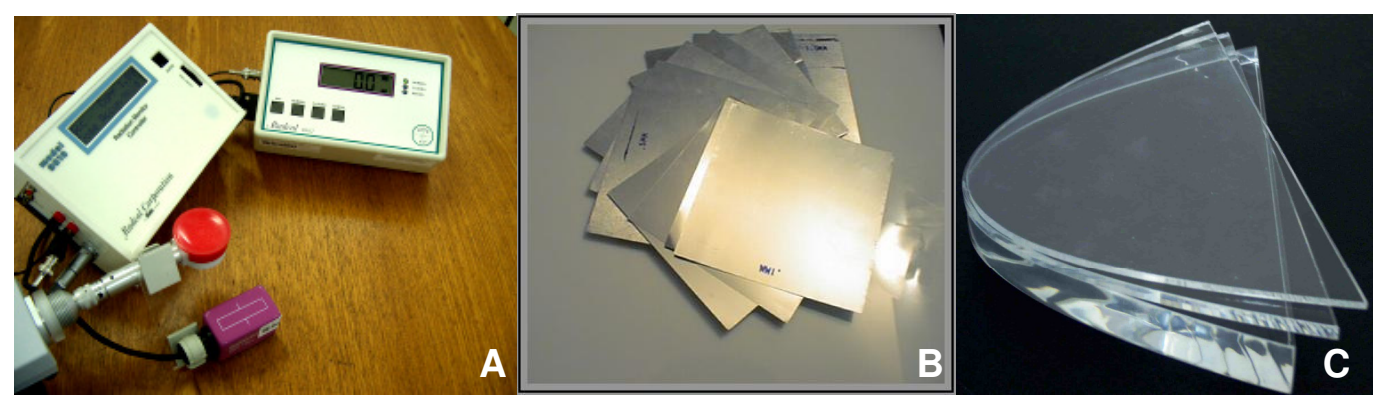

Figura 7 Instrumentos para a realização das medições. A. Conjunto Radcal composto por câmara de ionização de $6 \mathrm{~cm}^{3}$ dedicada a mamografia, monitor, medidor e sensor de tensão e tempo de exposição. B. Filtros de alumínio da Nuclear Associates. C. Manequim de PMMA com diferentes espessuras.

\subsection{Metodologia}

\subsubsection{Determinação da dimensão da amostra}

Para determinar a dimensão da amostra (n) necessária para validar o estudo realizado neste projeto, foi adotada a técnica de amostragem aleatória simples ${ }^{24}$.

Primeiramente calculou-se a média de exames realizados no período da coleta de dados nos três mamógrafos. O Hospital das Clínicas forneceu uma planilha com o número de exames realizados no departamento de mamografia em cada mês. Diante desta informação, determinou-se a média de exames realizados durante o período da coleta de dados, ou seja, em 4 meses, encontrando o valor igual a 6944. Este valor representa N, que é definido como a dimensão da população. 
Em seguida adotou-se um nível de confiança de $95 \%$, o que implica z igual a $1,96^{25}$. Além disso, foi considerado um erro amostral de $5 \%(e=0,05)$ e a estimativa inicial do desvio padrão igual a $50 \%\left(S_{0}=0,5\right)$.

Por fim, foi utilizada a expressão (11) para a determinação da dimensão da amostra e a expressão (12) para efetuar a correção do valor encontrado para a dimensão finita da população, chegando ao valor igual a 364 exames.

$$
n=\frac{(z)^{2} \cdot\left(S_{0}\right)^{2}}{e^{2}}(11) \quad n_{\text {corrigido }}=\frac{n \cdot N}{(n+N)}
$$

Apesar de ter sido encontrado o valor de $n$ igual a 364 exames, foi possível coletar 140 exames em cada mamógrafo, ou seja, 420 exames no total.

\subsubsection{Critérios de qualidade de imagem}

Para implementar os critérios de qualidade de imagem em mamografia de acordo com o documento European guidelines ${ }^{6}$ em um departamento de radiologia, foi preciso coletar dados durante um período de 4 meses. As imagens foram analisadas em duas projeções, na Crânio Caudal (CC) considerando 11 critérios de qualidade e na Médio Lateral Oblíqua (MLO) considerando 12 critérios de qualidade.

A metodologia adotada foi a de permanecer na sala durante a realização de cada exame para registrar as informações, nas Tabelas 6 (CC) e 7 (MLO), a respeito do equipamento, da combinação anodo-filtro, da tensão de aceleração de elétrons $(\mathrm{kVp})$, do produto corrente-tempo (mAs), da espessura das duas mamas de cada paciente, da técnica (operadora) que realizou o exame e da idade da paciente. Depois de processar a imagem, essa mesma técnica realizava uma avaliação, informando se os critérios de qualidade buscados estavam ou não presentes nas imagens. Conforme informação do radiologista responsável, as técnicas tinham total conhecimento para analisar essas imagens.

Nos casos em que a resposta era positiva, a imagem obtinha 1 (um) ponto. Se fosse negativa, era dada uma pontuação igual a 0 (zero). Cada imagem poderia obter um máximo de 11 pontos na CC e 12 pontos na MLO. 
Tabela 6 Tabela utilizada na coleta de dados dos exames na projeção Crânio Caudal.

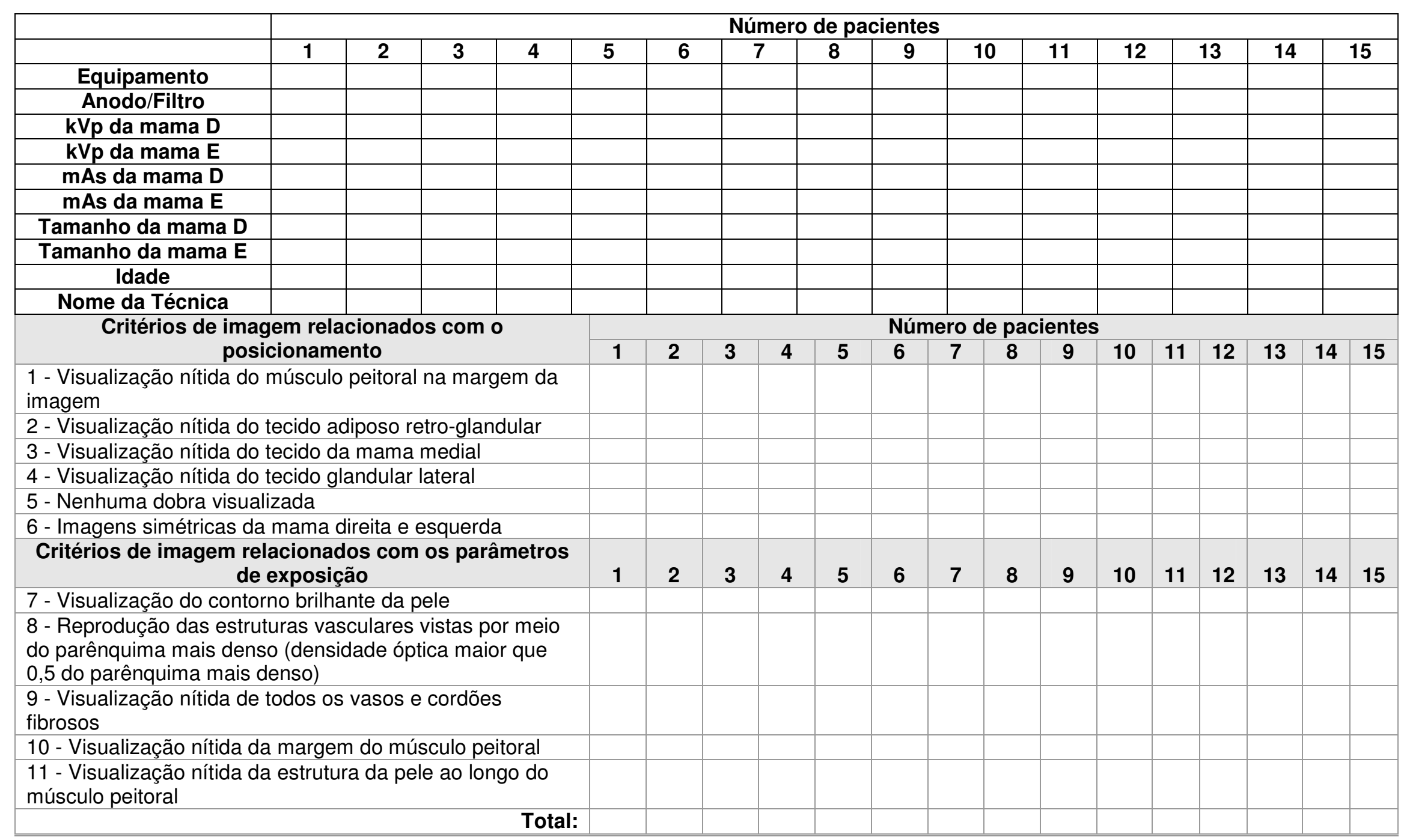


Tabela 7 Tabela utilizada na coleta de dados dos exames na projeção Médio Lateral Oblíqua.

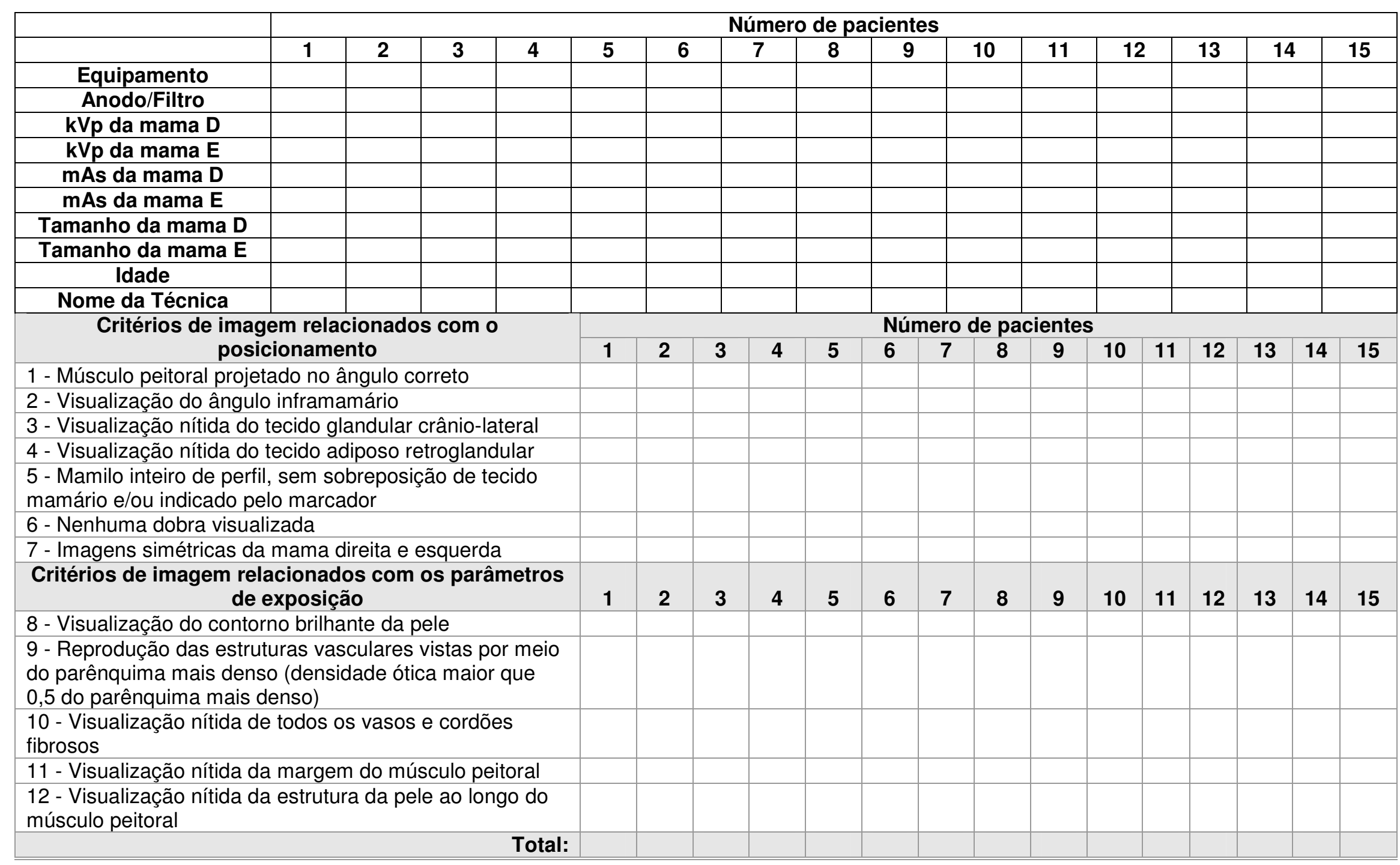




\subsubsection{Rejeição de imagem}

Durante o período de coleta de dados dos critérios de qualidade de imagem, foi observado que a maioria das imagens não atendia a todos os critérios. Portanto, em muitos casos, elas tinham que ser rejeitadas e, algumas vezes, a paciente era sujeita a uma exposição extra. Por isso, fez-se um estudo para detectar as principais causas de rejeição comparando-se os mamógrafos e verificando-se o índice de rejeição de cada equipamento.

Para a realização desse estudo foi necessário registrar quantos exames eram realizados em cada equipamento por dia, quantas imagens eram rejeitadas e determinar a sua causa. Os dados foram coletados durante 2 meses.

\subsubsection{Estimativa de dose na entrada da pele e dose glandular média}

Para estimar a dose na entrada da pele (DEP) e a dose glandular média (DGM) recebida por cada paciente durante um exame de mamografia em cada projeção, obteve-se a camada semi-redutora (CSR), o rendimento do tubo de raios $X$, o querma no ar, a tensão de aceleração de elétrons $(k V p)$ e o tempo de exposição de cada equipamento.

Primeiramente foi calculada a média da tensão de aceleração de elétrons $(\mathrm{kVp})$ e do produto corrente-tempo (mAs), baseando-se nas informações registradas durante a coleta de dados. Essas médias foram calculadas para cada espessura de mama comprimida, variando-se de $0,5 \mathrm{~cm}$ em um intervalo de 2,5 a $7,5 \mathrm{~cm}$, nas projeções CC e MLO, para cada combinação anodo-filtro em cada mamógrafo.

Adotando o método de Dance ${ }^{17,18}$ para estimar a DGM, foi medido o querma no ar selecionando a tensão e o produto corrente-tempo para cada espessura de mama comprimida em todas as combinações anodo-filtro de cada mamógrafo. Também foram feitas as medições com as placas de alumínio 1100 (99\% de pureza) para determinação da CSR em cada tensão de aceleração de elétrons. Com esses resultados, foi possível estimar também a DEP por meio do método do Guia da ANVISA ${ }^{13}$.

Para estimar a DGM recebida por cada paciente durante o exame de mamografia, adotou-se o método do TRS $457^{22}$ que se baseia na medição do 
rendimento do tubo em cada tensão de aceleração de elétrons para uma espessura de referência e calculou-se a DGM utilizando os mesmos fatores de correção apresentados por Dance ${ }^{17,18}$.

Seguindo o método de $\mathrm{Wu}^{19,20,21}$ a fim de obter uma estimativa da DGM, mediu-se o querma no ar com a câmara posicionada ao lado do manequim para cada espessura de mama comprimida das pacientes. Utilizando estes resultados, estimou-se a DEP pelo método do $\mathrm{ACR}^{16}$.

\subsubsection{Incertezas nas medidas}

A incerteza da medida é um parâmetro que caracteriza a dispersão dos valores que podem ser razoavelmente atribuídos ao mensurado. Ela é composta por dois tipos:

- $\quad$ Tipo A: são estimadas por métodos estatísticos para avaliar o grau de dispersão das medidas em relação ao valor médio;

- $\quad$ Tipo B: são estimadas por outros métodos que levam em consideração inúmeros fatores que não podem ser matematicamente determinados.

A incerteza do tipo A é calculada aplicando a equação 13:

$$
\sigma_{A}=\frac{\sigma}{\sqrt{n}}
$$

onde:

$\sigma=$ desvio padrão da medida

$n$ = número de medidas realizadas

O desvio padrão da medida é determinado pela equação 14:

$$
\sigma=\sqrt{\frac{\sum_{i=1}^{n}\left(x_{i}-\bar{x}\right)^{2}}{n-1}}
$$

onde:

$x_{i}=$ valor da medida

$\bar{x}=$ valor médio da medida

$n$ = número de medidas realizadas

Para a estimativa da incerteza tipo $\mathrm{B}\left(\sigma_{B}\right)$ foram considerados os erros provenientes da câmara de ionização, ou seja, o erro do coeficiente de calibração 
da câmara $(1,007 \pm 0,026)$, que é fornecido pelo Laboratório de Calibração do IPEN e do monitor da câmara $(0,0005)$, que foi considerado a metade da menor medida que o monitor apresenta.

A incerteza total das medidas foi estimada pela equação 15 :

$$
\sigma_{\text {Total }}=\sqrt{\left(\sigma_{A}{ }^{2}+{\sigma_{B}}^{2}\right)}
$$




\section{RESULTADOS E DISCUSSÕES}

\subsection{Controle de qualidade nos mamógrafos}

Em virtude dos testes de controle de qualidade realizados nos mamógrafos pode-se julgar o nível de confiança que se deve ter nos resultados obtidos neste projeto. Se algum dos mamógrafos apresentasse não-conformidade na maioria dos testes aplicados, não haveria garantia nas respostas dos estudos realizados neste trabalho.

É fundamental avaliar a constância do sistema elétrico, da qualidade da imagem e da processadora.

Portanto, antes de mostrar os resultados dos critérios de qualidade, índice de rejeição de imagem e estimativa de doses obtidos neste trabalho, é necessário se conhecer a resposta dos mamógrafos aos testes de controle de qualidade. Esses testes são realizados pela equipe da Seção Técnica de Aplicações em Diagnóstico por Imagem (STADI) no Instituto de Eletrotécnica e Energia da USP (IEE/USP). Os testes e os níveis de referência estabelecidos para cada teste estão apresentados na Tabela 8.

Os três mamógrafos apresentam conformidade na maioria dos testes realizados para o controle de qualidade. Um deles mostrou não conformidade na avaliação do contraste-detalhe, o outro apresentou não conformidade na avaliação do compressor da mama e da extensão do campo de radiação e o terceiro obteve resultado insatisfatório na avaliação da resolução.

A Tabela 8 não contempla os resultados dos testes de DEP, DGM e CSR realizados pela equipe da STADI. Estes parâmetros foram determinados, neste estudo, para várias espessuras de mama, se tratando da DEP e DGM e para vários valores de tensão de aceleração de elétrons, considerando a CSR. Portanto, serão apresentados ao longo deste trabalho, pois foram calculados e analisados para várias condições, além das exigidas no $A C R^{16}$ ou Guia da ANVISA $^{13}$.

A equipe de engenharia do hospital é informada sobre os resultados dos testes de controle de qualidade e providencia o conserto de todos os desajustes apresentados pelos equipamentos. Portanto, os equipamentos que apresentaram 
não conformidades foram imediatamente ajustados, com exceção do equipamento que apresentou resolução pequena, pois é uma limitação do próprio equipamento.

O Guia da ANVISA ${ }^{13}$ estabelece que a luminância do negatoscópio seja medida apenas na posição central. Porém a equipe da STADI realiza a medição nas extremidades também. Pode-se afirmar que todos os negatoscópios estão de conformidade com o Guia da ANVISA $^{13}$, porém $13 \%$ apresentam luminância inferior ao limite publicado no Guia em 1 das extremidades, $5 \%$ em 2 extremidades, $3 \%$ em 3 extremidades e $16 \%$ em 4 extremidades. Os monitores que são utilizados para o laudo dos exames realizados no equipamento digital não foram avaliados, pois não foram liberados para a realização do teste. Portanto, pode-se concluir destes resultados que a pequena luminância apresentada em alguns negatoscópios pode ter dificultado o radiologista que efetua o diagnóstico correto de alguns exames realizados nos equipamentos convencionais.

Foram detectados artefatos em todos os chassis quando foi realizado o teste para avaliação do contato tela-filme, mas a equipe de engenharia corrigiu esta não-conformidade imediatamente.

$\mathrm{Na}$ avaliação da câmara escura, foi constatado que a área da sala de câmara escura é menor que o recomendado pela Resolução SS-625/94 e foram encontradas frestas de luz em algumas áreas. 

Níveis de referência e recomendações do fabricante para testes de controle de qualidade em mamografia.

\begin{tabular}{|c|c|c|c|c|c|}
\hline \multicolumn{2}{|c|}{ Testes de Controle de Qualidade } & $\begin{array}{c}\text { Nível de referência Guia da } \\
\text { ANVISA }^{13}\end{array}$ & Nível de referência ACR $/ 99^{16}$ & $\begin{array}{l}\text { Recomendações do } \\
\text { fabricante }\end{array}$ & Outra referência \\
\hline \multicolumn{2}{|c|}{$\begin{array}{l}\text { Avaliação da diferença e da } \\
\text { reprodutibilidade do indicador de } \\
\text { tensão do tubo de raios } X\end{array}$} & $\pm 2,0 \mathrm{kVp}$ & $\leq 10 \%$ & (2) & - \\
\hline \multicolumn{2}{|c|}{$\begin{array}{c}\text { Avaliação da exatidão do tempo de } \\
\text { exposição }\end{array}$} & $\leq 10 \%$ & $\leq 10 \%$ & - & - \\
\hline \multicolumn{2}{|c|}{$\begin{array}{l}\text { Avaliação da reprodutibilidade do } \\
\text { tempo de exposição }\end{array}$} & $\leq 10 \%$ & $\leq 10 \%$ & - & - \\
\hline \multicolumn{2}{|c|}{$\begin{array}{l}\text { Avaliação da reprodutibilidade da } \\
\text { taxa de querma no ar }\end{array}$} & $\leq 10 \%$ & $\leq 10 \%$ & - & - \\
\hline \multicolumn{2}{|c|}{$\begin{array}{l}\text { Avaliação do rendimento do tubo } \\
\text { de raios } X\end{array}$} & - & $>7 \mathrm{mGy} / \mathrm{s}$ para $28 \mathrm{kVp}$ & - & - \\
\hline \multicolumn{2}{|c|}{$\begin{array}{l}\text { Avaliação da linearidade da taxa de } \\
\text { querma no ar com mAs }\end{array}$} & $\leq 20 \%$ & - & - & - \\
\hline \multirow{3}{*}{$\begin{array}{l}\text { Avaliação da } \\
\text { qualidade da } \\
\text { imagem }\end{array}$} & Manequim ACR & $\begin{array}{c}0,75 \mathrm{~mm} \text { (fibras) } \\
0,32 \mathrm{~mm} \\
\text { (microcalcificações) } \\
0,75 \mathrm{~mm} \text { (massas) }\end{array}$ & - & - & - \\
\hline & $\begin{array}{l}\text { Manequim - } \\
\text { avaliação do } \\
\text { mamógrafo } \\
\text { digital }\end{array}$ & 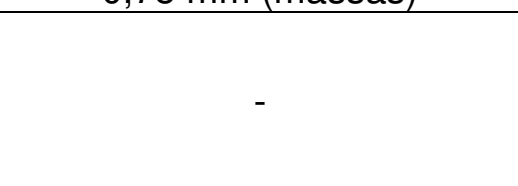 & $\begin{array}{c}0,54 \mathrm{~mm} \text { (fibras) } \\
0,24 \mathrm{~mm} \text { (microcalcificações) } \\
0,60 \mathrm{~mm} \text { (massas) }\end{array}$ & - & - \\
\hline & $\begin{array}{l}\text { Manequim } \\
\text { Contraste- } \\
\text { Detalhe }\end{array}$ & - & - & $\begin{array}{l}\text { Visualização de pelo } \\
\text { menos } 24 \text { objetos de } \\
49 \text { existentes }\end{array}$ & - \\
\hline \multicolumn{2}{|c|}{$\begin{array}{l}\text { Avaliação do sistema de } \\
\text { compressão da mama }\end{array}$} & $11 \mathrm{kgf} \leq \mathrm{m} \leq 18 \mathrm{kgf}$ & - & - & - \\
\hline \multirow{2}{*}{$\begin{array}{l}\text { Avaliação do } \\
\text { sistema de } \\
\text { colimação }\end{array}$} & $\begin{array}{c}\text { Coincidência de } \\
\text { campos }\end{array}$ & $\begin{array}{l}\text { Topo + P.T. } \leq 13,1 \\
\text { Esq. + Dir. } \leq 13,1\end{array}$ & $\begin{array}{c}\text { Topo + P.T. } \leq 13,1 \\
\text { Esq. + Dir. } \leq 13,1\end{array}$ & - & - \\
\hline & $\begin{array}{l}\text { Extensão do } \\
\text { campo de } \\
\text { radiação }\end{array}$ & $\begin{array}{l}\text { Parede torácica } \leq 3 \mathrm{~mm} \\
\text { Alinhamento } \leq 6,55 \mathrm{~mm}\end{array}$ & $\begin{array}{l}\text { Parede torácica } \leq 3 \mathrm{~mm} \\
\text { Outros lados } \leq 5 \mathrm{~mm} \\
\text { Alinhamento } \leq 6,55 \mathrm{~mm}\end{array}$ & - & - \\
\hline \multicolumn{2}{|c|}{ Avaliação da reprodutibilidade do } & $\leq 10 \%$ & $\leq 10 \%$ & - & - \\
\hline \multicolumn{2}{|c|}{$\begin{array}{l}\text { Avaliação do tamanho do ponto } \\
\text { focal }\end{array}$} & $\begin{array}{c}\text { Grosso } \leq 0,45 \\
\text { Fino } \leq 0,15\end{array}$ & - & $\begin{array}{l}\text { Grosso: } 0,3 \\
\text { Fino: } 0,1\end{array}$ & - \\
\hline
\end{tabular}




\begin{tabular}{|c|c|c|c|c|}
\hline Avaliação da compensação do AEC & $\begin{array}{c}\text { Variação da Densidade } \\
\text { Ótica } \leq 0,3\end{array}$ & $\begin{array}{c}\text { Variação da Densidade Ótica } \\
\leq 0,3\end{array}$ & - & - \\
\hline $\begin{array}{c}\text { Avaliação do controle automático } \\
\text { de densidades }\end{array}$ & $\begin{array}{l}\text { Variação da Densidade } \\
\text { Ótica } \leq 0,2\end{array}$ & $\begin{array}{c}\text { Variação do mAs: } \\
12 \% \text { à } 15 \% \\
\text { Variação da Densidade Ótica: } \\
0,13 \text { a } 0,17 \\
\end{array}$ & - & - \\
\hline $\begin{array}{l}\text { Avaliação da resolução do } \\
\text { equipamento }\end{array}$ & - & $\begin{array}{l}\text { paralelo: } 13 \text { pares de linha } / \mathrm{mm} \\
\text { perpendicular: } 11 \text { pares de } \\
\text { linha/mm }\end{array}$ & - & - \\
\hline Luminância do negatoscópio & $\begin{array}{c}\text { Entre } 3000 \text { e } 3500 \mathrm{~cd} / \mathrm{m}^{2} \\
\text { Variação da luminosidade } \\
\leq 15 \%\end{array}$ & - & - & - \\
\hline $\begin{array}{l}\text { Avaliação do sistema de } \\
\text { processamento da imagem }\end{array}$ & - & - & - & Linha de base \\
\hline $\begin{array}{l}\text { Verificação do movimento da grade } \\
\text { anti-espalhamento }\end{array}$ & - & - & - & - \\
\hline $\begin{array}{l}\text { Avaliação da razão Contraste- } \\
\text { Ruído }\end{array}$ & - & - & - & - \\
\hline $\begin{array}{c}\text { Medida da Função de Transferência } \\
\text { de Modulação }\end{array}$ & - & - & $\begin{array}{l}2,09 \mathrm{pl} / \mathrm{mm}: \\
>58 \% \\
3,93 \mathrm{pl} / \mathrm{mm}: \\
>25 \%\end{array}$ & - \\
\hline $\begin{array}{l}\text { Avaliação da Uniformidade do } \\
\text { campo horizontal }\end{array}$ & - & - & - & - \\
\hline Avaliação de Artefatos & - & Ausência de artefatos & - & - \\
\hline $\begin{array}{l}\text { Verificação da temperatura da } \\
\text { câmara escura }\end{array}$ & - & - & $10^{\circ} \mathrm{C} \leq \mathrm{T} \leq 24^{\circ} \mathrm{C}$ & - \\
\hline $\begin{array}{l}\text { Verificação da umidade relativa da } \\
\text { câmara escura }\end{array}$ & - & - & $30-50 \%$ & - \\
\hline $\begin{array}{l}\text { Verificação da validade dos } \\
\text { químicos }\end{array}$ & - & - & - & $\begin{array}{l}\text { Não devem estar vencidos } \\
\text { (Portaria MS 453/98) }\end{array}$ \\
\hline Verificação da validade dos filmes & - & - & - & $\begin{array}{l}\text { Não devem estar vencidos } \\
\text { (Portaria MS 453/98) }\end{array}$ \\
\hline $\begin{array}{l}\text { Verificação do sistema de } \\
\text { reciclagem de químicos }\end{array}$ & - & - & - & $\begin{array}{c}\text { Revelador e fixador não } \\
\text { devem ser liberados em } \\
\text { aterros } \\
\text { RDC } 306 / 2004\end{array}$ \\
\hline $\begin{array}{l}\text { Verificação da posição de } \\
\text { armazenamento dos filmes }\end{array}$ & - & & - & $\begin{array}{c}\text { Posição vertical } \\
\text { (Portaria MS 453/98) }\end{array}$ \\
\hline
\end{tabular}




\begin{tabular}{|c|c|c|c|c|}
\hline $\begin{array}{l}\text { Verificação do processamento dos } \\
\text { filmes de mamografia }\end{array}$ & - & - & - & $\begin{array}{c}\text { Processadora deve ser } \\
\text { dedicada } \\
\text { (Portaria MS 453/98) }\end{array}$ \\
\hline $\begin{array}{l}\text { Verificação da localização da } \\
\text { câmara escura }\end{array}$ & - & - & - & $\begin{array}{c}\text { Filmes devem estar } \\
\text { afastados de fontes de } \\
\text { radiação } \\
\text { (Portaria MS 453/98) }\end{array}$ \\
\hline $\begin{array}{c}\text { Verificação da área da câmara } \\
\text { escura }\end{array}$ & - & - & - & $\begin{array}{c}\geq 5 \mathrm{~m}^{2} \\
\text { SS-625/94 }\end{array}$ \\
\hline $\begin{array}{l}\text { Verificação do posicionamento do } \\
\text { interruptor de luz clara }\end{array}$ & - & - & - & $\begin{array}{l}\text { Evitar acionamento acidental } \\
\text { (Portaria MS 453/98) }\end{array}$ \\
\hline $\begin{array}{c}\text { Verificação do sistema de exaustão } \\
\text { de gases e vapores }\end{array}$ & - & - & - & $\begin{array}{l}\text { Deve possuir o sistema } \\
\text { (Portaria MS 453/98) }\end{array}$ \\
\hline $\begin{array}{l}\text { Verificação do tipo de piso da } \\
\text { câmara escura }\end{array}$ & - & - & - & $\begin{array}{c}\text { Anticorrosivo, impermeável e } \\
\text { antiderrapante } \\
\text { (Portaria MS 453/98) }\end{array}$ \\
\hline $\begin{array}{l}\text { Verificação do revestimento das } \\
\text { paredes da câmara escura }\end{array}$ & - & - & - & $\begin{array}{l}\text { Revestimento deve ser } \\
\text { resistente aos químicos } \\
\text { (Portaria MS 453/98) }\end{array}$ \\
\hline $\begin{array}{l}\text { Verificação das condições de } \\
\text { limpeza da câmara escura }\end{array}$ & - & - & - & $\begin{array}{c}\text { Mantida limpa } \\
\text { (Portaria MS 453/98) }\end{array}$ \\
\hline $\begin{array}{c}\text { Verificação da vedação da câmara } \\
\text { escura }\end{array}$ & - & - & - & $\begin{array}{c}\text { Deve ter vedação especial } \\
\text { contra luz do dia ou artificial } \\
\text { (Portaria MS 453/98) }\end{array}$ \\
\hline $\begin{array}{c}\text { Verificação do filtro de água da } \\
\text { processadora }\end{array}$ & - & - & $\begin{array}{l}\text { Água deve ser } \\
\text { filtrada }\end{array}$ & - \\
\hline $\begin{array}{c}\text { Verificação do tempo de } \\
\text { manipulação seguro da câmara } \\
\text { escura }\end{array}$ & - & - & - & $\begin{array}{c}\geq 60 s \\
\text { IEC 61223-2-3/93 }\end{array}$ \\
\hline
\end{tabular}




\subsection{Critérios de qualidade de imagem}

As respostas dos mamógrafos que atendem a cada critério de qualidade da imagem nas projeções CC e MLO estão apresentadas na Figura 8.
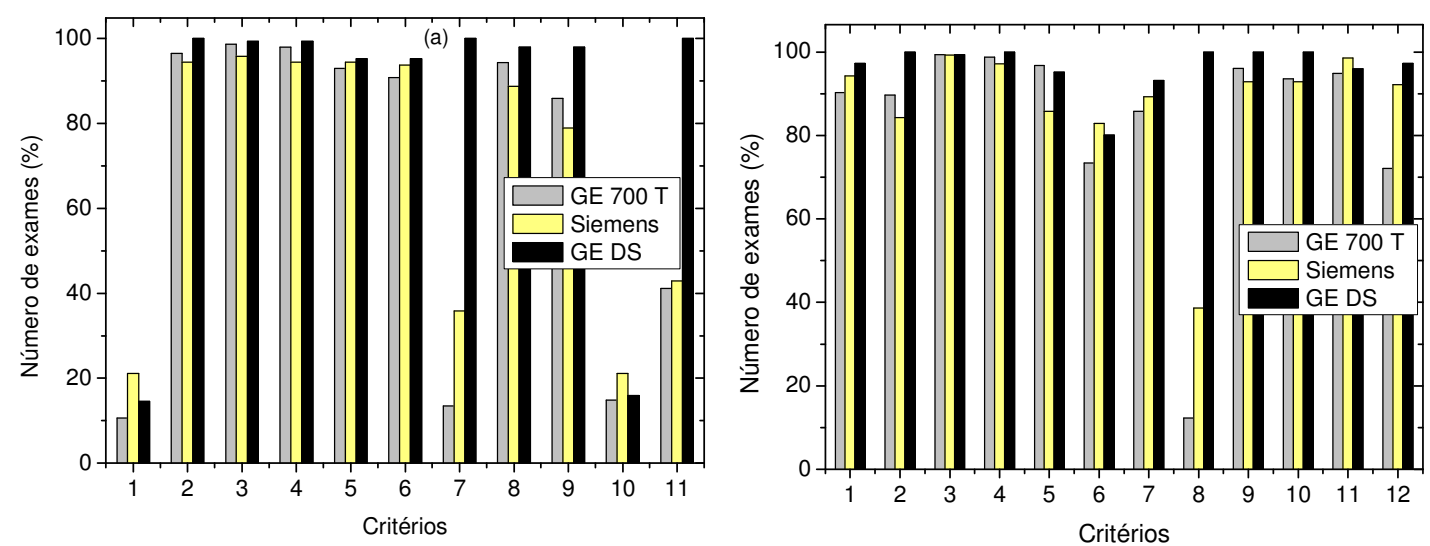

Figura 8 Porcentual dos exames que atendem a cada critério de qualidade na projeção (a) CC e (b) MLO nos três mamógrafos.

Na projeção $\mathrm{CC}$, tanto o critério 1 (visualização nítida do músculo peitoral na margem da imagem) quanto o critério 10 (visualização nítida da margem do músculo peitoral) estão presentes em $\pm 20 \%$ ou abaixo desse valor nas imagens dos três equipamentos. Para poder visualizar, nas imagens, o músculo peitoral na margem da imagem e a margem do músculo é necessário que a técnica (operadora) alongue bastante a mama da paciente para que toda a mama seja posicionada no suporte. Muitas pacientes não conseguem deixar a técnica posicionar toda a mama na mesa em virtude da dor que sentem e em outros casos, a paciente não apresenta condições para que a técnica consiga atender a esse critério, como por exemplo, pacientes que apresentam saliência na coluna vertebral.

Os critérios 7 (visualização do contorno brilhante da pele) e 11 (visualização nítida da estrutura da pele ao longo do músculo peitoral) apresentaram concordância de $15 \%$ e $40 \%$, respectivamente, nas imagens do equipamento GE $700 \mathrm{~T}$, em torno de $40 \%$ do Siemens e $100 \%$ do GE DS. Esse resultado mostra que o equipamento GE DS apresenta uma faixa dinâmica que permite, em todas as imagens, visualizar a estrutura e o contorno brilhante da pele. Por outro lado, a maioria das imagens de ambos os equipamentos convencionais não permite 
visualizar essas estruturas, fazendo com que o diagnóstico se torne duvidoso, pois a visualização dessas estruturas é fundamental para garantir que toda a mama esteja sendo visualizada na imagem.

Os critérios 5 (nenhuma dobra visualizada) e 6 (imagens simétricas da mama direita e esquerda) estão presentes em aproximadamente $90 \%$ das imagens nos três equipamentos. Esses critérios estão associados ao posicionamento da paciente e dependem da técnica. Em alguns casos, não é possível atender a esses critérios, quando a paciente apresenta mamas assimétricas ou operadas.

Em todos os outros critérios da projeção CC o equipamento GE DS apresenta melhores resultados que os convencionais, já que não depende da processadora.

Na projeção MLO, o critério 8 (Visualização do contorno brilhante da pele) é equivalente ao critério 7 da projeção CC e apresenta a mesma resposta, ou seja, está presente em $15 \%$ das imagens no GE 700 T, em $40 \%$ no Siemens e em $100 \%$ no GE DS. A justificativa do bom desempenho do equipamento GE DS é que a faixa dinâmica permite uma boa qualidade da imagem. O critério 12 (Visualização nítida da estrutura da pele ao longo do músculo peitoral), apesar de ser semelhante ao critério 11 da CC, apresentou melhores resultados nos equipamentos convencionais, porém o mamógrafo GE DS continuou apresentando os critérios em $100 \%$ das imagens. O motivo para essa melhoria na resposta pode ser explicado pelo fato de na imagem da projeção MLO aparecer mais músculo que na imagem da CC, portanto o contraste é maior, sendo possível visualizar mais facilmente a estrutura da pele.

Os critérios 6 (nenhuma dobra visualizada), equivalente ao critério 5 da CC e 0 7 (imagens simétricas da mama direita e esquerda), equivalente ao 6 da CC, apresentam resultados um pouco inferiores aos apresentados na outra projeção. Como esse critério depende do posicionamento da paciente, essa redução no resultado se justifica pelo fato de ser mais difícil o posicionamento da paciente na projeção MLO.

O critério 1 está presente em mais de 90 \% das imagens dos mamógrafos. Este critério também depende do posicionamento da paciente, ou seja, da técnica e da colaboração da paciente.

A diferença de resultado entre os equipamentos convencionais e o digital está em torno de $10 \%$ ao analisar o critério 2 (visualização do ângulo inframamário). 0 
equipamento GE DS atende a este critério em $100 \%$ das imagens por causa da faixa dinâmica.

Nos critérios restantes, os mamógrafos obtiveram respostas semelhantes.

Em ambas as projeções notou-se que o equipamento GE DS apresenta uma faixa dinâmica que permite serem observadas todas as estruturas que se deseja nas imagens, enquanto que nas imagens dos equipamentos convencionais não foi possível visualizar algumas estruturas fundamentais nas imagens, por causa da menor faixa dinâmica. Essa diferença de faixa dinâmica deve ser associada à processadora que é necessária nos equipamentos convencionais, enquanto que é dispensada nos digitais. O equipamento deve ser ajustado em função das condições da processadora para conseguir o melhor contraste possível na imagem.

\subsection{Rejeição de Imagem}

Como mostra a Tabela 9, a principal causa de rejeição de imagem, neste departamento, é causado pelo mau posicionamento da paciente (29\%). Muitas vezes a paciente não colabora com o posicionamento, pois sente dor ou apresenta limitações para a realização do exame.

Em seguida, um outro fator importante de rejeição (14 \%) é a seleção dos parâmetros da irradiação. Esta falha ocorre principalmente em mamas operadas e com a presença de silicone. Para minimizar a rejeição de imagem provocada por essas causas é aconselhável um treinamento intensivo às técnicas, já que a paciente, nestes casos, terá que repetir a imagem se submetendo a uma exposição de radiação extra.

Uma causa de rejeição que também se destacou foi o filme revelado sem exposição (12\%), porém só se aplica aos equipamentos convencionais e ocorre em virtude dos testes na processadora, falha do equipamento de raios $\mathrm{X}$ e falta de atenção da técnica. Isto leva ao desperdício de filme e de produtos químicos e pode ser minimizado por meio de treinamento para as técnicas.

Outra causa frequente (10\%) é o filme totalmente velado. Esta perda de filme ocorre em consequência da dupla exposição do mesmo filme. Esta falha pode ser minimizada realizando treinamento para as técnicas, já que esta falha provoca uma exposição extra à paciente. 
Tabela 9 Principais causas de rejeição de imagem detectadas nos 3 mamógrafos.

\begin{tabular}{c|c}
\hline \hline Causas de rejeição de imagem & Porcentagem (\%) \\
\hline Posicionamento errado da paciente & 29,0 \\
Parâmetros errados & 14,0 \\
Filme revelado sem exposição & 12,0 \\
Filme totalmente velado & 10,0 \\
Falha técnica da câmara escura / chassi & 9,0 \\
Falha da processadora & 6,0 \\
Testes durante a manutenção & 5,0 \\
Movimento da paciente & 4,0 \\
Fotocélula inadequada & 3,0 \\
Falha do equipamento & 2,0 \\
Uso desnecessário do colimador & 2,0 \\
Identificação errada dos dados da paciente & 1,0 \\
Filme não revelado & 1,0 \\
Falta do marcador na mama & 1,0 \\
Imagens potencialmente aproveitáveis & 1,0 \\
\hline \hline
\end{tabular}

Falha técnica na câmara escura ou do chassi foi motivo para 9 \% de rejeição. Dentre essas falhas pode-se citar a presença de cabelo e marca de unha no chassi e revelação de dois filmes colados. Esta falha também pode ser minimizada realizando-se treinamento para os operadores da processadora.

A falha da processadora é responsável pela rejeição de $6 \%$ das imagens. Para evitar problemas com ela, basta fazer um controle maior de todos os fatores que influenciam o bom funcionamento.

Os testes que são realizados nos equipamentos durante a manutenção são responsáveis por $5 \%$ das imagens rejeitadas. Para reduzir esta rejeição, é necessário conscientizar os técnicos de manutenção.

Algumas pacientes se movimentam durante a exposição de raios $X$, o que provoca uma rejeição de $4 \%$ das imagens.

A escolha inadequada da fotocélula ocorre em $3 \%$ das imagens rejeitadas. Escolhendo a fotocélula inadequada para uma exposição, a imagem, depois de revelada, não apresenta o contraste necessário para o bom diagnóstico, pois o equipamento vai fornecer uma exposição também inadequada. Essa falha ocorre 
como consequência da falta de atenção por parte das técnicas. Este erro pode ser minimizado por meio da realização de treinamento para estas trabalhadoras.

Existem falhas no equipamento, que representam $2 \%$ da perda de imagem. Algumas vezes o equipamento interrompe a exposição durante a realização do exame, portanto, a técnica tem que realizar uma nova exposição, expondo a paciente novamente à radiação. Para minimizar essas falhas, deve-se aumentar a frequência da manutenção preventiva nos equipamentos.

Também rejeitando $2 \%$ das imagens, outra causa é o uso desnecessário do colimador. Essa falha também é causada pela falta de atenção da técnica e pode ser minimizada por meio de treinamento.

A identificação errada dos dados da paciente é uma causa exclusiva do equipamento GE DS e é responsável pela rejeição de $1 \%$ das imagens. Ao se registrar alguma informação errônea dos dados da paciente no software do equipamento GE DS, a técnica é obrigada a repetir o exame, fazendo com que a paciente esteja sujeita a uma exposição extra, apenas para corrigir uma informação. Essa falha ocorre por falta de atenção da técnica, mas pode ser solucionada com a melhoria do software utilizado, permitindo a alteração dos dados da paciente antes de imprimir a imagem.

Os filmes não revelados são responsáveis por $1 \%$ das imagens rejeitadas. Esta perda de filme ocorre em virtude da falha por parte do técnico da câmaraescura ao fechar o porta-chassi depois de ter colocado o chassi dentro. Esta falha pode ser minimizada realizando treinamento para os técnicos da câmara-escura.

Em alguns casos, a técnica realiza a exposição na paciente para aquisição da imagem e não coloca o marcador nos sinais que a paciente apresenta. A técnica é obrigada a repetir essa exposição, pois o radiologista pode confundir este sinal com outra estrutura. Para minimizar esta falha também é aconselhável o treinamento das técnicas.

Por fim, são rejeitadas, com $1 \%$ do total das imagens, as radiografias denominadas potencialmente aproveitáveis. Às vezes a técnica radiografa a paciente mais vezes que o necessário por achar que o radiologista pedirá um complemento, mas nem sempre este pedido ocorre, portanto a imagem é rejeitada.

O índice de rejeição de imagem por equipamento foi de $5 \%$ nos equipamentos convencionais e de $3 \%$ no GE DS. Esse resultado mostra que apesar da 
diferença ser pequena, os sistemas convencionais rejeitam mais imagens que o digital. O equipamento GE DS poderia rejeitar menos imagens, pois ele não depende da processadora e apresenta recursos do software para a melhoria da imagem, porém, como a aquisição da imagem é fácil e rápida, muitas vezes a técnica repete uma imagem, que já apresentava as características fundamentais para o diagnóstico, pois ela acredita que aquela imagem poderia ser ainda melhor.

\subsection{Estimativa da dose na entrada da pele e da dose glandular média}

\subsubsection{Desenvolvimento do manequim}

Para medir o querma no ar e estimar a dose glandular baseando-se no método de $W u^{19,20,21}$, é necessário um manequim para espalhar a radiação. Portanto, foi desenvolvido, por meio de um projeto do Centro de Metrologia das Radiações do Instituto de Pesquisas Energéticas e Nucleares (IPEN/CNEN), um objeto (Figura 7 C) composto por placas de PMMA, com diferentes espessuras para simular as diferentes dimensões da mama comprimida das pacientes nas projeções CC e MLO. Foi considerado que estas placas de acrílicos apresentam coeficiente de atenuação similar ao de uma mama com $50 \%$ de tecido glandular e $50 \%$ de tecido adiposo, pois existe uma relação de equivalência entre o acrílico e o tecido da mama $^{18}$.

\subsubsection{Determinação da camada semi-redutora}

Foi necessário determinar os valores da camada semi-redutora para cada combinação anodo-filtro nos três mamógrafos estudados para a estimativa da DEP, DGM e para atender a um dos testes de controle de qualidade. Todos os valores encontrados, apresentados na Tabela 10, encontram-se dentro dos limites estabelecidos pelo Guia do $\mathrm{ACR}^{16}$. Para uma melhor comparação entre os valores das camadas semi-redutoras obtidos nas diferentes combinações anodo-filtro, presentes em cada equipamento, foi elaborado o gráfico apresentado na Figura 9. 
Analisando este gráfico, percebe-se o mesmo comportamento nas respostas, à medida que aumenta a tensão de aceleração de elétrons, os valores das camadas semi-redutoras também aumentam.

Tabela 10 Valores de camada semi-redutora (mmAl) para cada tensão de aceleração de elétrons $(k V p)$ calculados nas diferentes combinações anodo-filtro nos três mamógrafos estudados.

\begin{tabular}{|c|c|c|c|c|c|}
\hline \multirow{2}{*}{$\begin{array}{c}\text { Tensão } \\
(\mathbf{k V p})\end{array}$} & \multicolumn{5}{|c|}{ Valores de camada semi-redutora (mmAl) } \\
\hline 23 & GE 700T & Siemens Mo/Mo & Siemens Mo/Rh & GE DS Mo/Rh & GE DS Rh/Rh \\
\hline 24 & 0,29 & 0,31 & 0,36 & - & - \\
\hline 25 & 0,30 & 0,32 & 0,37 & - & - \\
\hline 26 & 0,33 & 0,33 & 0,39 & - & - \\
\hline 27 & 0,34 & 0,34 & 0,40 & 0,38 & - \\
\hline 28 & 0,35 & 0,35 & 0,41 & 0,39 & 0,38 \\
\hline 29 & 0,35 & 0,36 & 0,41 & 0,40 & 0,40 \\
\hline 30 & 0,36 & 0,37 & 0,42 & 0,41 & 0,41 \\
\hline 31 & 0,37 & 0,38 & 0,43 & - & 0,42 \\
\hline 32 & 0,38 & 0,38 & 0,43 & 0,42 & 0,43 \\
\hline
\end{tabular}

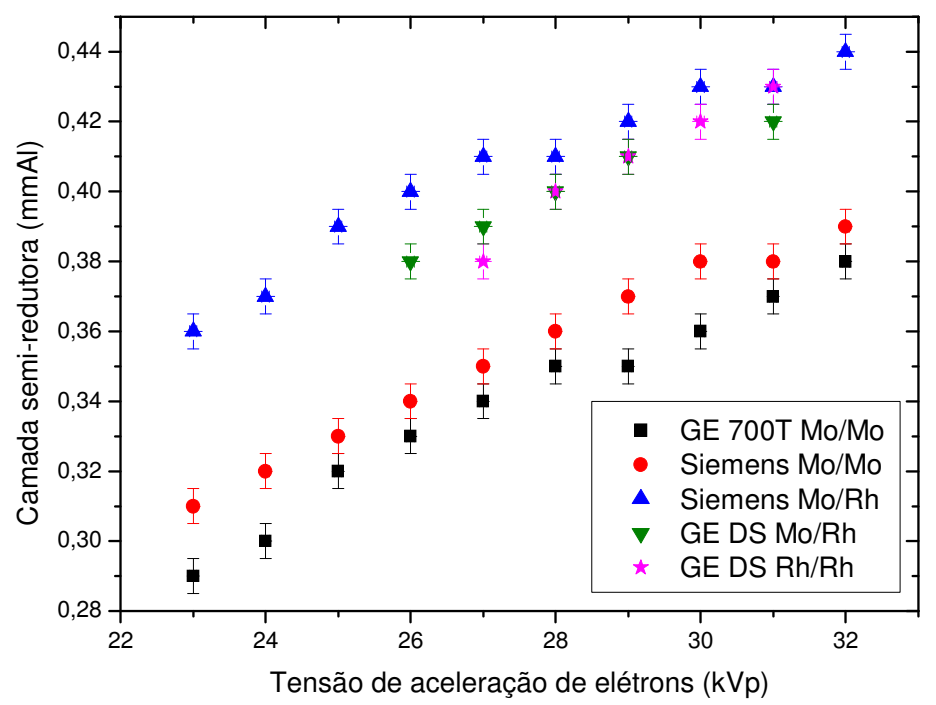

Figura 9 Comparação entre os valores de CSR obtidos para cada tensão de aceleração de elétrons $(k V p)$ nas diferentes combinações anodo-filtro em cada mamógrafo. 
Realizando a comparação entre os resultados obtidos em cada combinação anodo-filtro dos mamógrafos, percebe-se que a combinação Mo/Mo, em ambos os equipamentos, possui valores de CSR menores do que Mo/Rh e Rh/Rh, para todas as tensões medidas. Os valores das duas combinações estudadas no GE DS são bem próximos, inclusive dos 5 pontos medidos, 2 são exatamente iguais. A combinação $\mathrm{Mo} / \mathrm{Rh}$ do equipamento Siemens apresenta valores de CSR maiores que os valores apresentados em todas as outras combinações. Essas variações de valores apresentadas pelas combinações em cada equipamento devem ser associadas ao fato de que 0 tubo de raios $X$ de cada equipamento apresenta diferentes rendimentos para cada combinação anodo-filtro.

\subsubsection{Dose na entrada da pele}

A dose na entrada da pele foi calculada para diferentes espessuras de mama comprimida, baseando-se no método do Guia da ANVISA ${ }^{13}$ e no Guia do $\mathrm{ACR}^{16}$, nos equipamentos: GE $700 \mathrm{~T}$, na combinação anodo-filtro Mo-Mo, Siemens, nas combinações Mo/Mo e Mo/Rh e GE DS, nas combinações Mo/Rh e Rh/Rh.

Foram realizadas duas comparações entre os valores encontrados:

- Comparação 1: entre os resultados de dose nas projeção CC e MLO para uma mesma espessura de mama da paciente.

- Comparação 2: entre os resultados de dose nas projeções CC e MLO para uma mesma paciente, que é definido no gráfico como "Projeção MLO por paciente".

\subsubsection{Método do Guia da ANVISA}

As Figuras 10, 11, 12, 13 e 14 ilustram os resultados de dose na entrada da pele estimados por este método. 


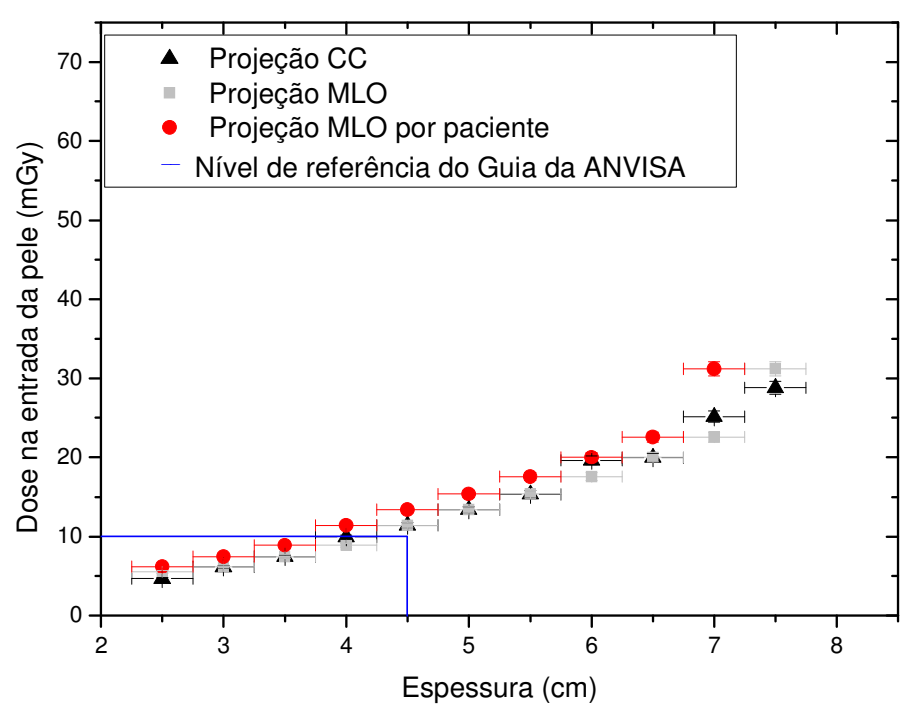

Figura 10 Dose na entrada da pele para cada espessura de mama comprimida estudada na projeção CC e MLO no equipamento GE 700 T.

- $\quad$ Comparação 1:

- Equipamento GE $700 \mathrm{~T}$ :

- Combinação anodo-filtro Mo/Mo (Figura 10): percebe-se que há diferença de dose $\leq 1 \mathrm{mGy}$ em $73 \%$ dos resultados de dose e 1 $m G y<\Delta$ DEP $\leq 2,5$ mGy em $27 \%$;

Equipamento Siemens :

- Combinação anodo-filtro Mo/Mo (Figura 11): há diferença $\leq 1 \mathrm{mGy}$ em $11 \%, 1 \mathrm{mGy}<\Delta$ DEP $\leq 2,5 \mathrm{mGy}$ em $22 \%, 2,5 \mathrm{mGy}<\Delta$ DEP $\leq 4$ mGy em $\quad 44 \%, 4$ mGy $<\Delta$ DEP $\leq 6$ mGy em $33 \%$ e 6 mGy $<\Delta$ DEP $\leq 10$ mGy em $11 \%$ dos casos;

- Combinação anodo-filtro Mo/Rh (Figura 12): há diferença 1 mGy < $\Delta$ DEP $\leq 2,5$ mGy em $29 \%, 2,5$ mGy $<\Delta$ DEP $\leq 4$ mGy em $43 \%, 4$ mGy $<\Delta$ DEP $\leq 6$ mGy em $14 \%$ e 6 mGy $<\Delta$ DEP $\leq 10$ mGy em $14 \%$ dos resultados; 


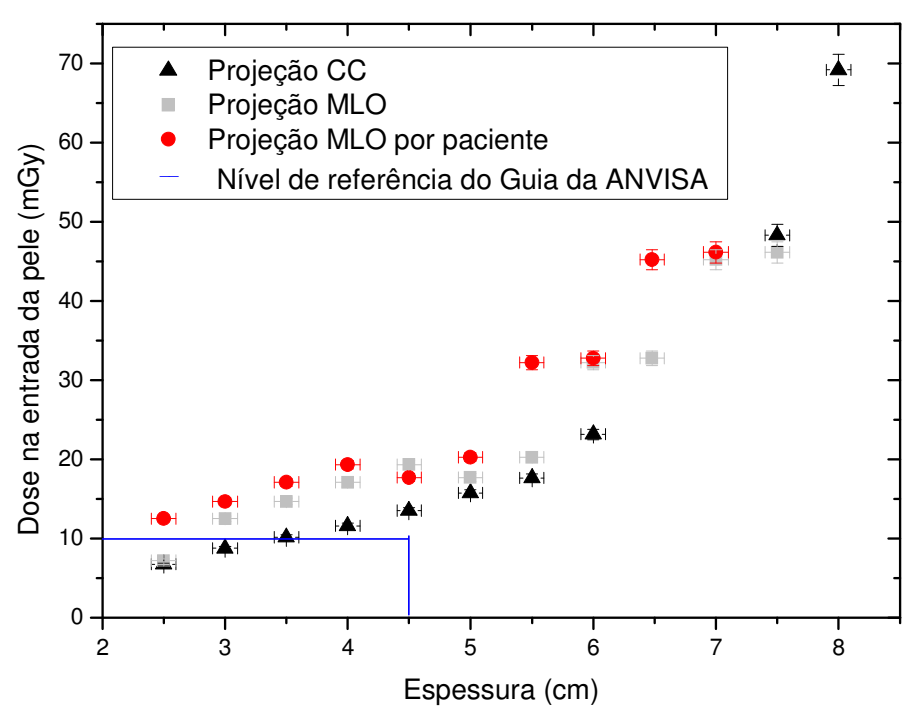

Figura 11 Dose na entrada da pele para cada espessura de mama comprimida estudada na projeção CC e MLO no equipamento Siemens, combinação Mo/Mo.

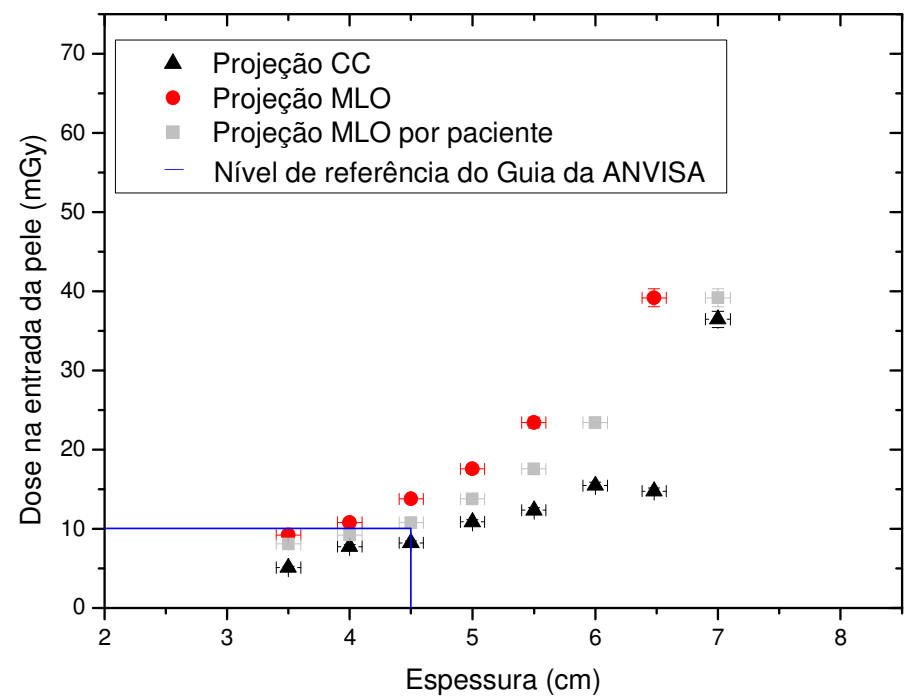

Figura 12 Dose na entrada da pele para cada espessura de mama comprimida estudada na projeção CC e MLO no equipamento Siemens, combinação Mo/Rh. 


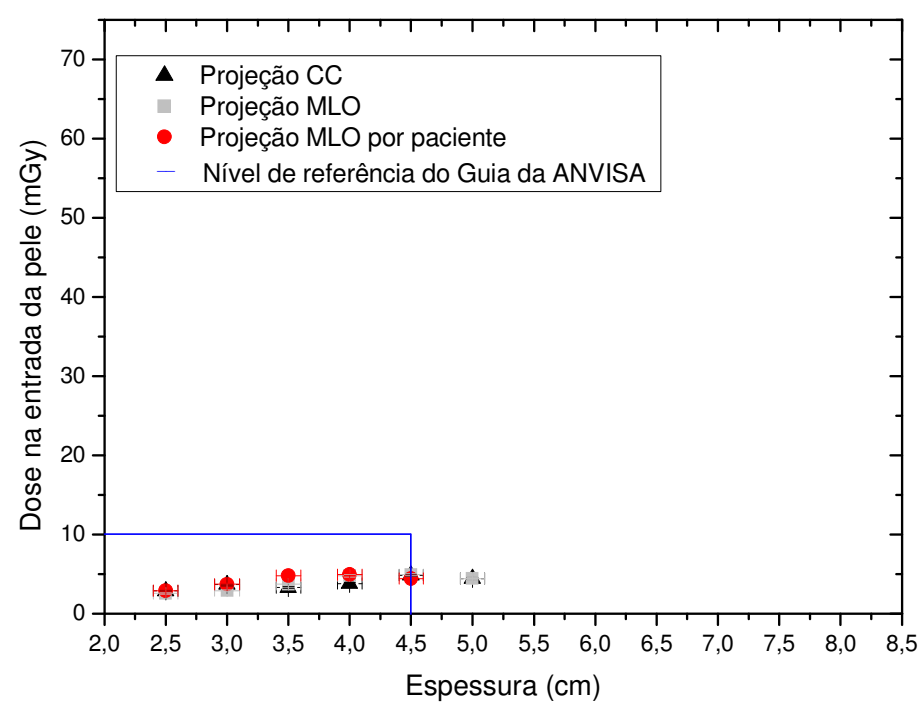

Figura 13 Dose na entrada da pele para cada espessura de mama comprimida estudada na projeção CC e MLO no equipamento GE DS, combinação Mo/Rh.

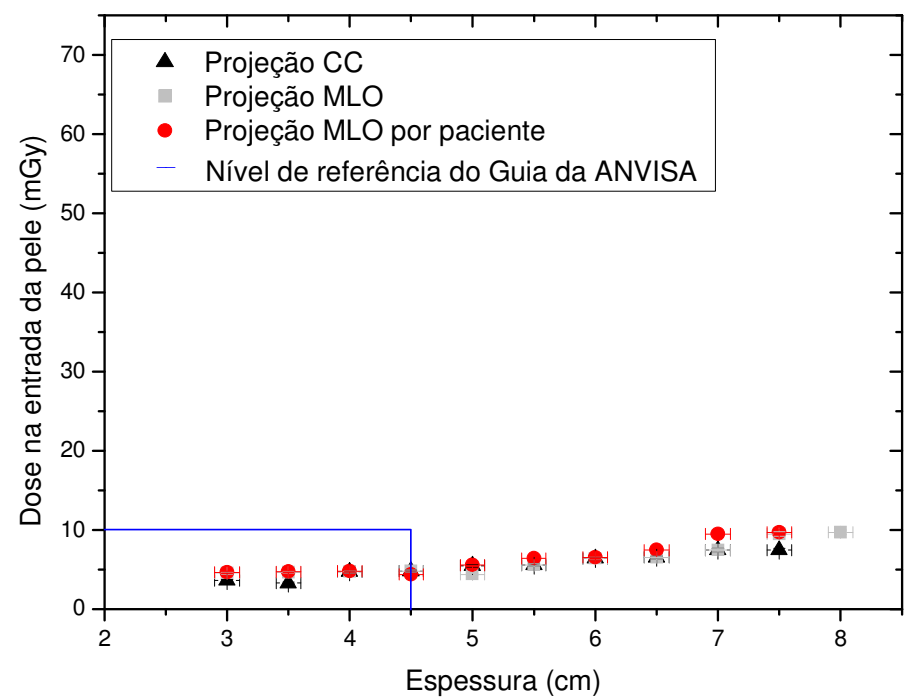

Figura 14 Dose na entrada da pele para cada espessura de mama comprimida estudada na projeção CC e MLO no equipamento GE DS, combinação Rh/Rh.

- Equipamento GE DS:

- Combinação anodo-filtro Mo/Rh (Figura 13): observa-se que em $100 \%$ dos resultados a diferença é $\leq 1$ mGy; 
- Combinação anodo-filtro Rh/Rh (Figura 14): em 80 \% a diferença é $\leq 1 \mathrm{mGy}$ e em $20 \%$ dos resultados a diferença é $1 \mathrm{mGy}<\Delta \mathrm{DEP} \leq$ 2,5 mGy.

- $\quad$ Comparação 2:

- Equipamento GE $700 \mathrm{~T}$ :

- Combinação anodo-filtro Mo/Mo (Figura 10): uma diferença $\leq 1$ mGy em $10 \%$ dos resultados, 1 mGy $<\Delta$ DEP $\leq 2,5$ mGy em $80 \%$ e igual a 6 mGy em $10 \%$;

- Equipamento Siemens:

- Combinação anodo-filtro Mo/Mo (Figura 11): diferença 4 mGy < $\Delta$ DEP $\leq 6 \mathrm{mGy}$ em $50 \%, 6 \mathrm{mGy}<\Delta \mathrm{DEP} \leq 10 \mathrm{mGy}$ em $30 \%$ e $10 \mathrm{mGy}<\Delta \mathrm{DEP} \leq 14,5 \mathrm{mGy}$ em $20 \%$ dos resultados;

- Combinação anodo-filtro Mo/Rh (Figura 12): que há diferença 2,5 mGy $<\Delta$ DEP $\leq 4$ mGy em $17 \%, 4$ mGy $<\Delta$ DEP $\leq 6$ mGy em $32 \%, \quad 6$ mGy $<\Delta$ DEP $\leq 10$ mGy em $17 \%, 10$ mGy $<\Delta$ DEP $\leq \quad 14,5$ mGy em $17 \%$ e 14,5 mGy $<\Delta$ DEP $\leq 24,5$ mGy em $17 \%$;

- Equipamento GE DS:

- Combinação anodo-filtro Mo/Rh (Figura 13): que há diferença $\leq$ 1 mGy em $60 \%$ e 1 mGy $<\Delta$ DEP $\leq 2,5$ mGy em $40 \%$ dos resultados;

- Combinação anodo-filtro $\mathrm{Rh} / \mathrm{Rh}$ (Figura 14): há diferença $\leq 1$ mGy em $70 \%$ e 1 mGy $<\Delta$ DEP $\leq 2,5$ mGy em $30 \%$ dos resultados.

A diferença de espessura de mama comprimida para uma mesma paciente entre as duas projeções é de aproximadamente $0,5 \mathrm{~cm}$ maior na MLO, por isso a dose nesta projeção também é maior. Este aumento de espessura pode ser explicado pela presença de grande parte do músculo peitoral na projeção MLO.

O nível de referência determinado pelo método do Guia da ANVISA ${ }^{13}$ para DEP em uma mama padrão $(4,5 \mathrm{~cm})$ é $10 \mathrm{mGy}$. Assim, no equipamento GE $700 \mathrm{~T}$ (Figura 10), a mama comprimida na projeção CC e MLO ultrapassou $14 \%$ do nível de referência. No equipamento Siemens, na combinação anodo-filtro Mo/Mo 
(Figura 11), na projeção CC, o valor de dose ultrapassou o nível de referência em $35 \%$; na MLO, ultrapassou $93 \%$. Neste mesmo equipamento, mas na combinação anodo-filtro Mo/Rh (Figura 12), os valores de dose obtidos na projeção CC estão abaixo do nível de referência, na projeção MLO os valores ultrapassaram o nível $8 \%$. Os valores de dose obtidos no equipamento GE DS, em ambas as projeções e em ambas as combinações anodo-filtro apresentadas nas Figuras 13 e 14, estão abaixo do nível de referência.

\subsubsection{Método do ACR}

Todas as medições foram realizadas com o manequim, baseando-se no método apresentado pelo American College of Radiology (ACR $)^{16}$.

As Figuras 15, 16, 17, 18 e 19 mostram a comparação de dose entre a projeção CC e a MLO em cada espessura e para uma mesma paciente.

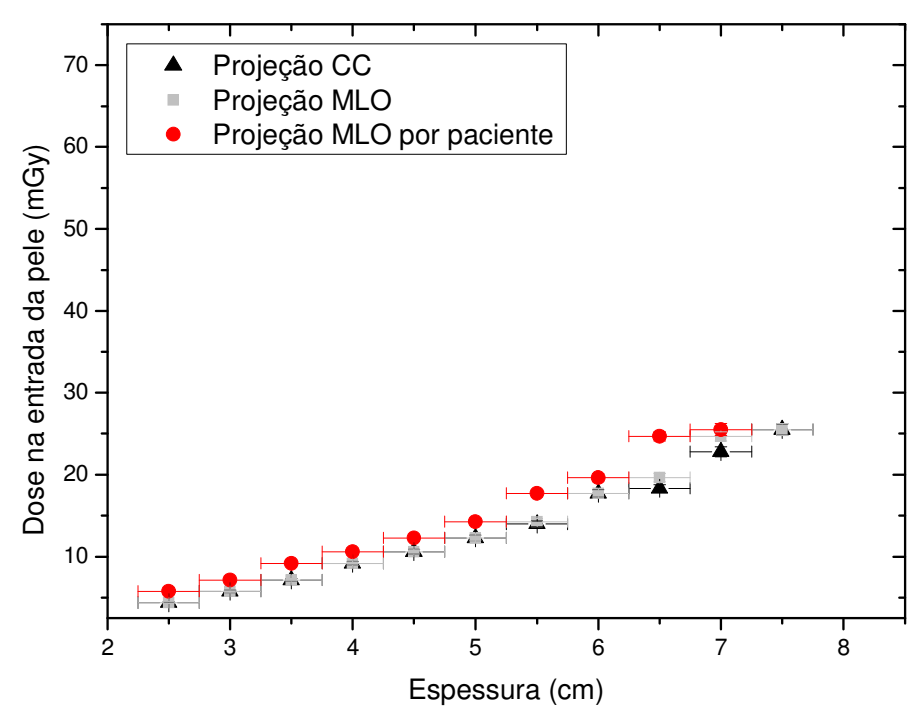

Figura 15 Dose na entrada da pele para cada espessura de mama comprimida estudada da projeção CC e MLO no equipamento GE $700 \mathrm{~T}$. 




Figura 16 Dose na entrada da pele para cada espessura de mama comprimida estudada da projeção CC e MLO no equipamento Siemens - MoMo.

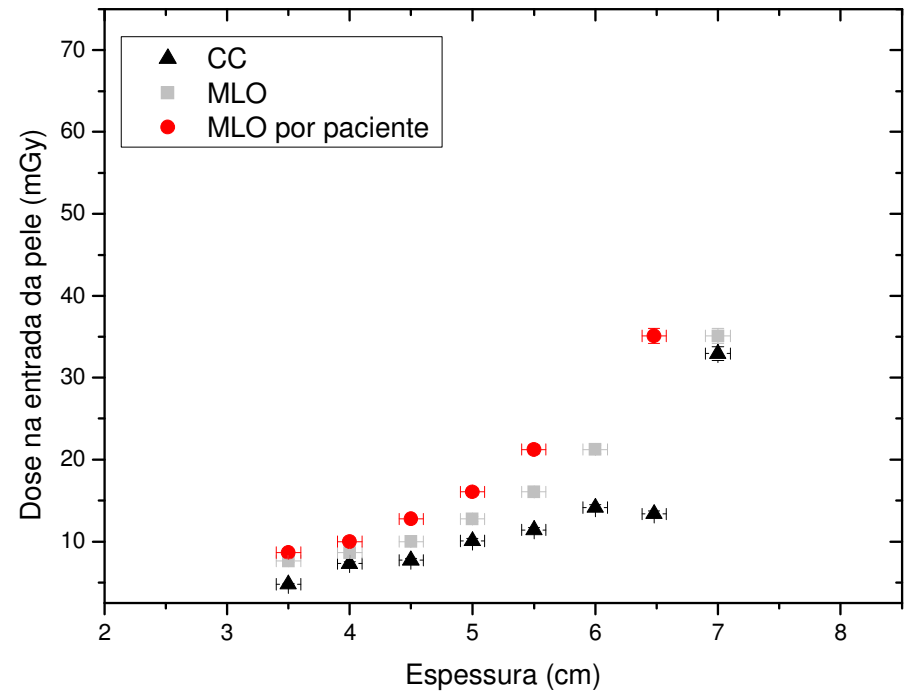

Figura 17 Dose na entrada da pele para cada espessura de mama comprimida estudada da projeção CC e MLO no equipamento Siemens - MoRh. 


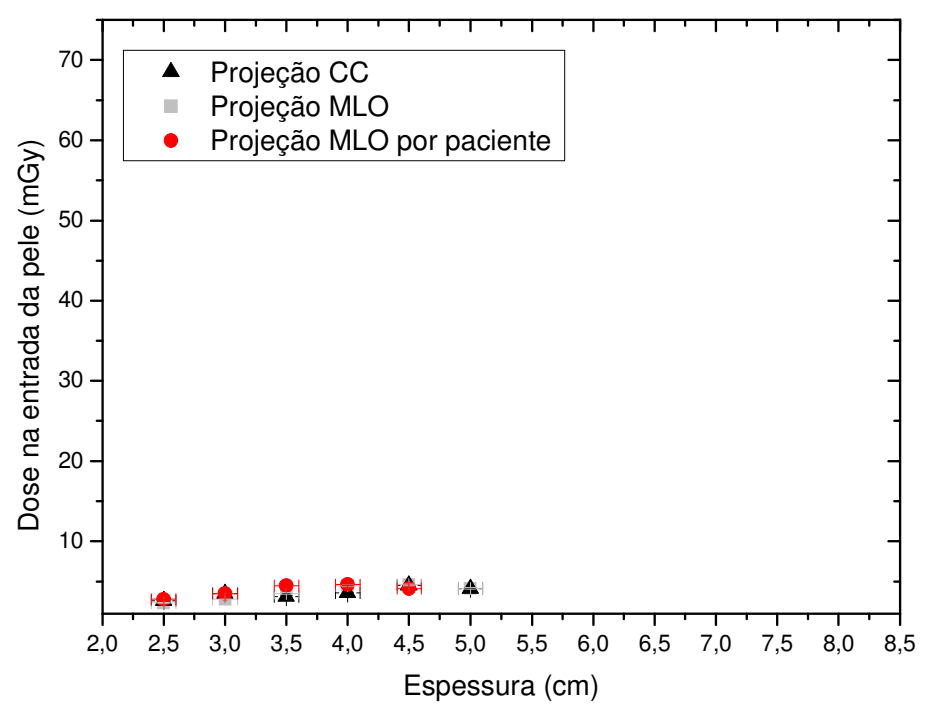

Figura 18 Dose na entrada da pele para cada espessura de mama comprimida estudada da projeção CC e MLO no equipamento GE DS - MoRh.

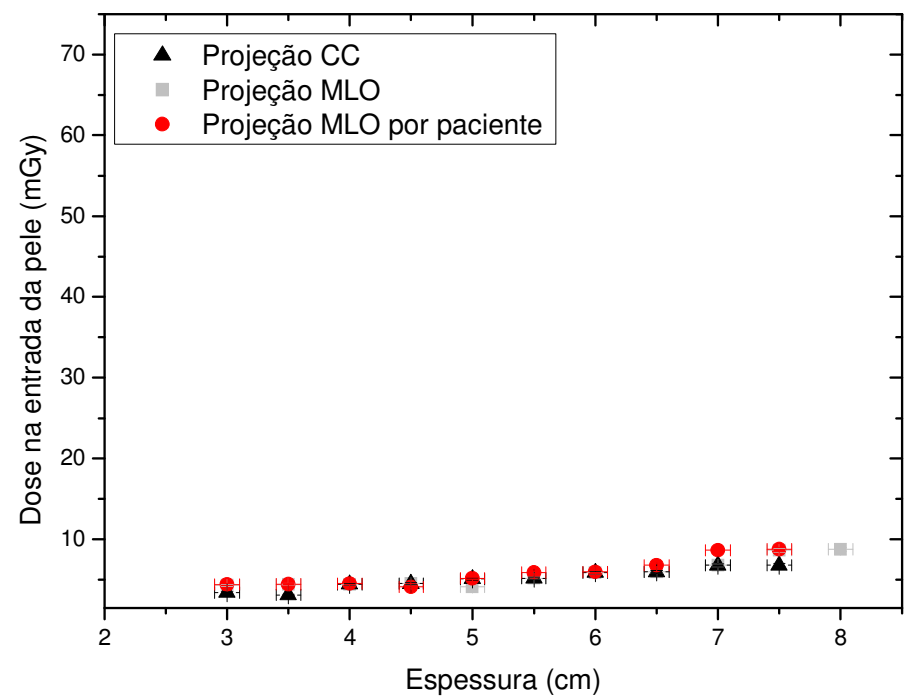

Figura 19 Dose na entrada da pele para cada espessura de mama comprimida estudada da projeção CC e MLO no equipamento GE DS - RhRh.

A diferença de dose entre os valores foi calculada em porcentagem e podem ser observadas na Tabela 11. 
Tabela 11 Diferença de DEP (\%) estimada pelo método do $\mathrm{ACR}^{16}$ para uma mesma espessura de mama comprimida e para uma mesma paciente para as diferentes combinações anodo-filtro nos três mamógrafos.

\begin{tabular}{c|c|c|c}
\hline \hline \multicolumn{4}{c}{ GE 700T Mo/Mo (Figura 15) } \\
\hline \hline & Mesma espessura & \multicolumn{2}{c}{ Mesma paciente } \\
$\%$ & Diferença de doses (mGy) & $\%$ & Diferença de doses (mGy) \\
\hline \hline 82 & 0 a 1 & 80 & 1 a 2,5 \\
18 & 1 a 2,5 & 10 & 2,5 a 4 \\
& & 10 & 4 a 6 \\
\hline
\end{tabular}

\begin{tabular}{c|c|cc}
\hline \hline \multicolumn{4}{c}{ Siemens Mo/Mo (Figura 16) } \\
\hline \hline & Mesma espessura & \multicolumn{3}{c}{ Mesma paciente } \\
$\%$ & Diferença de doses (mGy) & $\%$ & Diferença de doses (mGy) \\
\hline \hline 11 & 0 a 1 & 12,5 & 2,5 a 4 \\
33 & 1 a 2,5 & 37,5 & 4 a 6 \\
11 & 2,5 a 4 & 37,5 & 6 a 10 \\
33 & 4 a 6 & 12,5 & 10 a 14,5 \\
11 & 6 a 10 & & \\
\hline
\end{tabular}

\begin{tabular}{c|c|cc}
\hline \hline \multicolumn{4}{c}{ Siemens Mo/Rh (Figura 17) } \\
\hline \hline \multicolumn{3}{c|}{ Mesma espessura } & \multicolumn{2}{c}{ Mesma paciente } \\
$\%$ & Diferença de doses (mGy) & $\%$ & Diferença de doses (mGy) \\
\hline \hline 43 & 1 a 2,5 & 40 & 2,5 a 4 \\
29 & 2,5 a 4 & 40 & 4 a 6 \\
14 & 4 a 6 & 20 & 6 a 10 \\
14 & 6 a 10 & & \\
\hline
\end{tabular}

\begin{tabular}{c|c|c|c|}
\hline \hline \multicolumn{4}{c}{ GE DS Mo/Rh (Figura 18) } \\
\hline \hline \multicolumn{3}{c|}{ Mesma espessura } & \multicolumn{3}{c}{ Mesma paciente } \\
$\%$ & Diferença de doses (mGy) & $\%$ & Diferença de doses (mGy) \\
\hline \hline 100 & 0 a 1 & 60 & 0 a 1 \\
& & 40 & 1 a 2,5
\end{tabular}

\begin{tabular}{c|c|c|c}
\hline \hline \multicolumn{4}{c}{ GE DS Rh/Rh (Figura 19) } \\
\hline \hline & Mesma espessura & \multicolumn{2}{c}{ Mesma paciente } \\
\hline$\%$ & Diferença de doses (mGy) & $\%$ & Diferença de doses (mGy) \\
\hline \hline 80 & 0 a 1 & 70 & 0 a 1 \\
20 & 1 a 2,5 & 30 & 1 a 2,5 \\
\hline
\end{tabular}

Para este método não é possível verificar se os resultados estão ou não dentro do nível de referência, pois o $\mathrm{ACR}^{16}$ não apresenta nível de referência de dose para a DEP. 


\subsubsection{Comparação entre os métodos}

Para comparar os resultados de dose na entrada da pele estimados pelos dois métodos estudados, pode-se visualizar as Figuras 20, 22, 24, 26 e 28, na Projeção CC e as Figuras 21 23, 25, 27 e 29, na Projeção MLO.

Analisando o equipamento GE 700 T, na projeção CC (Figura 20), observa-se que os valores de dose na entrada da pele estimados pelo método do Guia da $\mathrm{ANVISA}^{13}$ são sempre maiores que os estimados pelo método do $A C R^{16}$. À medida que a espessura de mama comprimida aumenta, a diferença entre as doses estimadas pelos diferentes métodos também aumenta. Os resultados de dose na projeção MLO (Figura 21) são um pouco diferentes dos apresentados na projeção CC, pois o método do Guia da ANVISA $^{13}$ apresenta valores de dose maiores em $73 \%$ dos pontos de medição, ou seja, das espessuras de mama comprimida estudadas. Em ambas as projeções, a diferença entre os valores de dose estimados em cada método não ultrapassa 2 mGy, com exceção dos valores obtidos na espessura de 7 e $7,5 \mathrm{~cm}$.

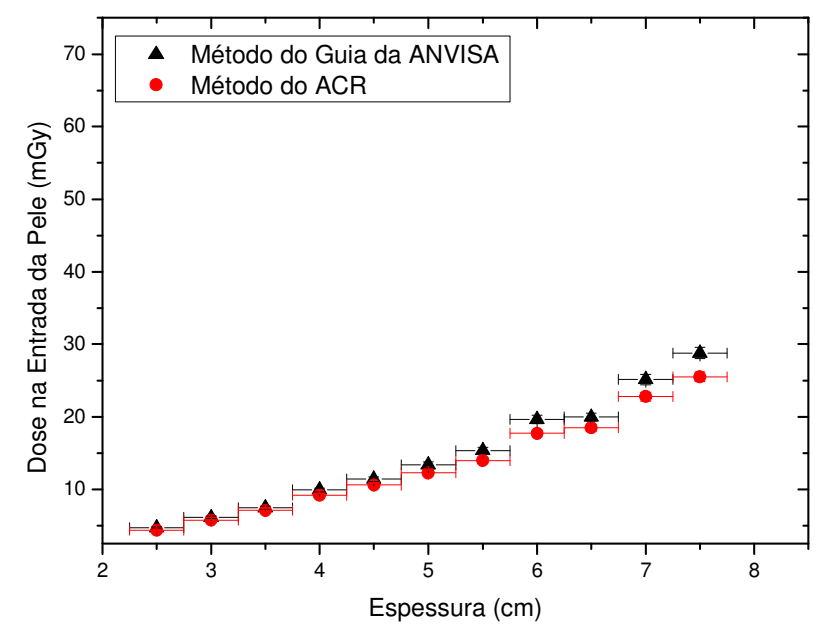

Figura 20 Comparação entre as estimativas de DEP calculada pelos dois métodos diferentes para cada espessura de mama comprimida estudada na projeção CC no equipamento GE $700 \mathrm{~T}$. 


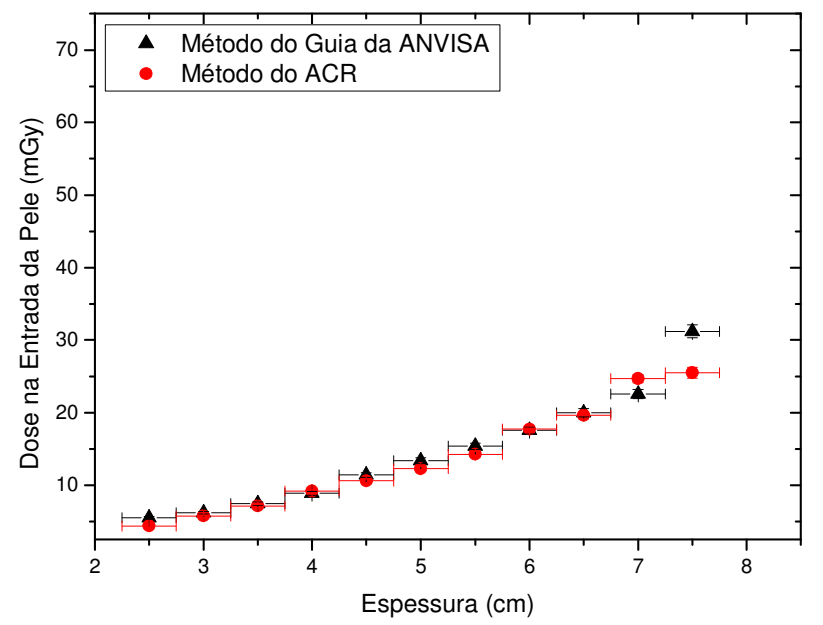

Figura 21 Comparação entre as estimativas de DEP calculada pelos dois métodos diferentes para cada espessura de mama comprimida estudada na projeção MLO no equipamento GE $700 \mathrm{~T}$.

Os valores de dose estimados pelo método da $\mathrm{ANVISA}^{13}$ são maiores que os estimados pelo método do $\mathrm{ACR}^{16}$ em $100 \%$ dos dados obtidos nas duas projeções do equipamento Siemens, na combinação anodo-filtro Mo/Mo (Figuras 22 e 23).

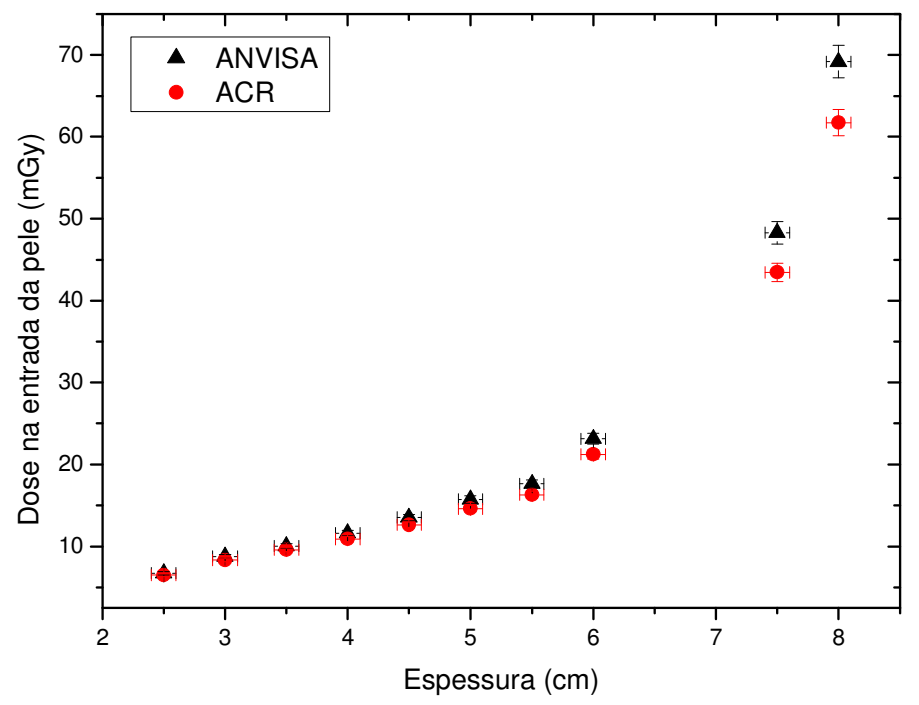

Figura 22 Comparação entre as estimativas de DEP calculada pelos dois métodos diferentes para cada espessura de mama comprimida estudada na projeção CC no equipamento Siemens - MoMo. 
A diferença entre os valores de dose aumenta à medida que a espessura aumenta, não passando de 5 mGy na CC e 4 mGy na MLO.

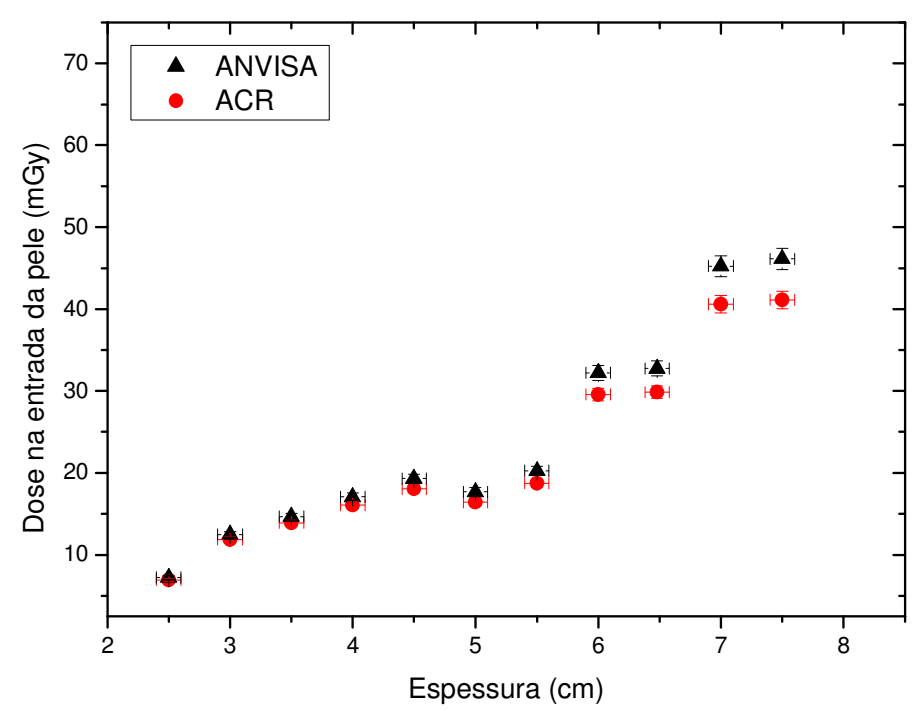

Figura 23 Comparação entre as estimativas de DEP calculada pelos dois métodos diferentes para cada espessura de mama comprimida estudada na projeção MLO no equipamento Siemens - MoMo.

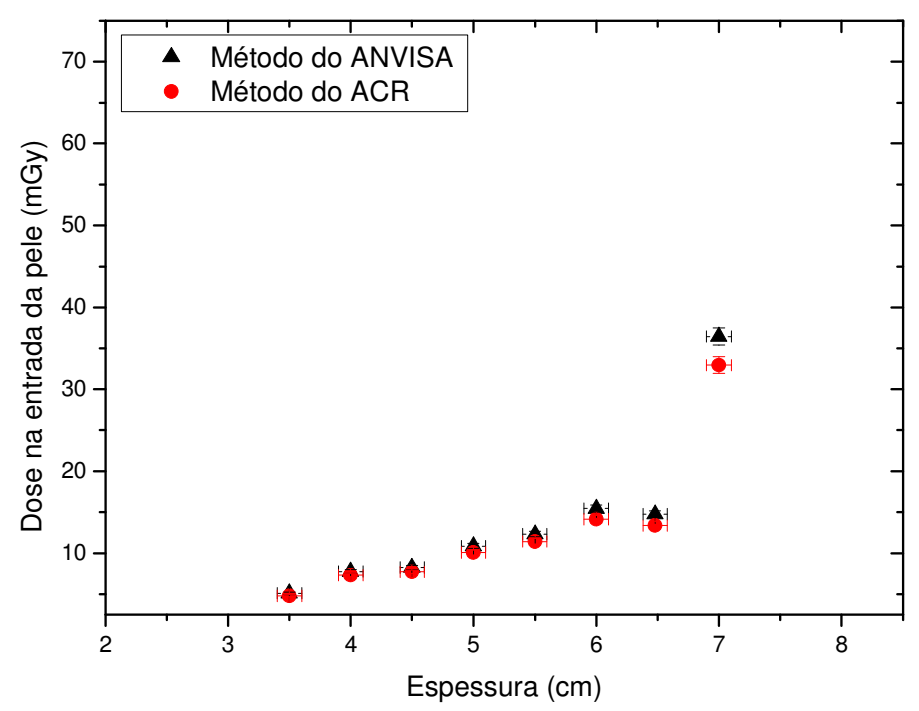

Figura 24 Comparação entre as estimativas de DEP calculada pelos dois métodos diferentes para cada espessura de mama comprimida estudada na projeção CC no equipamento Siemens - MoRh. 
No equipamento Siemens com a combinação anodo-filtro $\mathrm{Mo} / \mathrm{Rh}$, na projeção CC (Figura 24), os valores estimados pelo método da $\mathrm{ANVISA}^{13}$ são sempre maiores que os estimados pelo método do $A C R^{16}$, porém esta diferença não ultrapassa $1,5 \mathrm{mGy}$, com exceção da espessura $7 \mathrm{~cm}$ que essa diferença é aproximadamente 3,5 mGy.

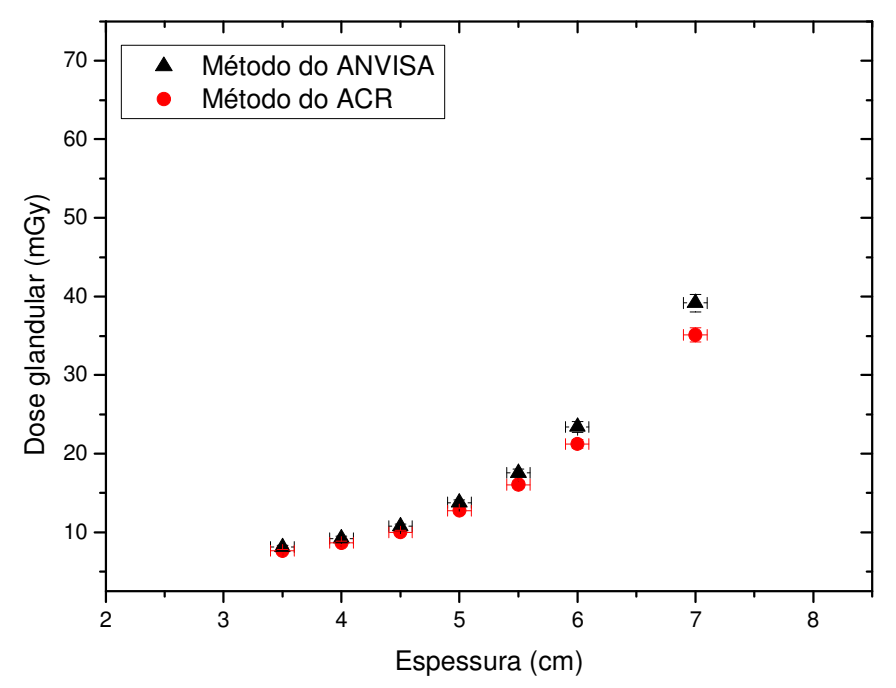

Figura 25 Comparação entre as estimativas de DEP calculada pelos dois métodos diferentes para cada espessura de mama comprimida estudada na projeção MLO no equipamento Siemens - MoRh.

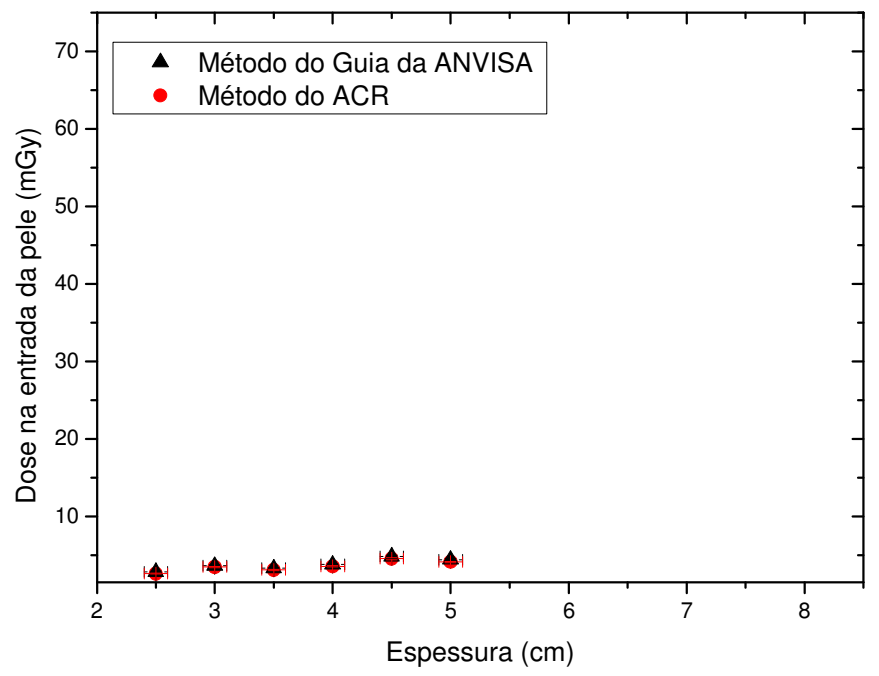

Figura 26 Comparação entre as estimativas de DEP calculada pelos dois métodos diferentes para cada espessura de mama comprimida estudada na projeção CC no equipamento GE DS - MoRh. 
Na projeção MLO (Figura 25) também se observa que os valores estimados pelo método da $A N V I S A^{13}$ são maiores que os estimados no método do $A_{C R}{ }^{16} \mathrm{e}$ esta diferença não ultrapassa $2,5 \mathrm{mGy}$, com exceção da espessura $7 \mathrm{~cm}$, onde esta diferença é aproximadamente 4 mGy.

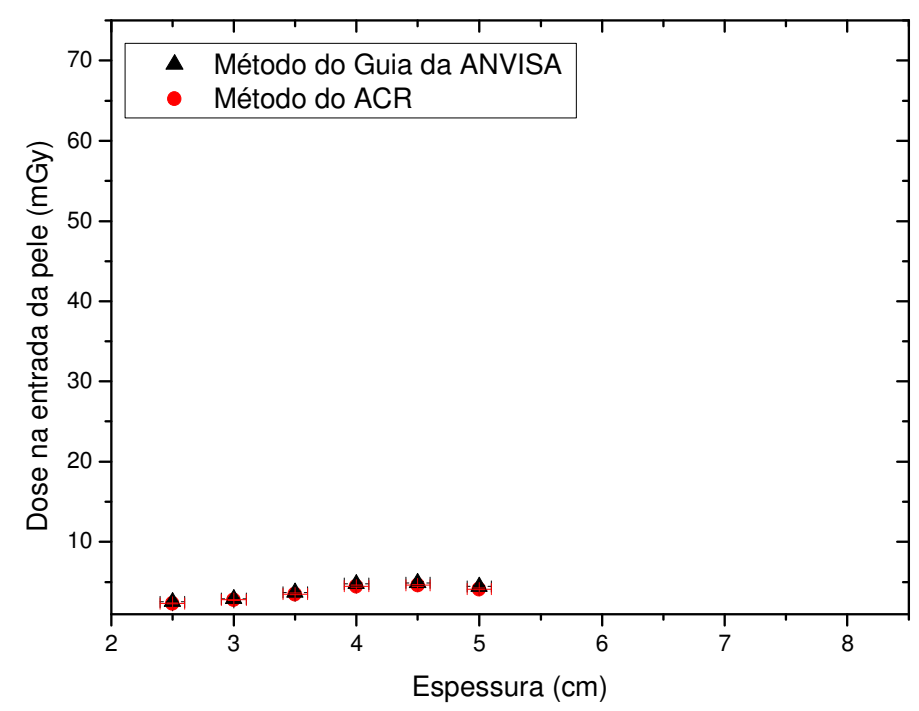

Figura 27 Comparação entre as estimativas de DEP calculada pelos dois métodos diferentes para cada espessura de mama comprimida estudada na projeção MLO no equipamento GE DS - MoRh.

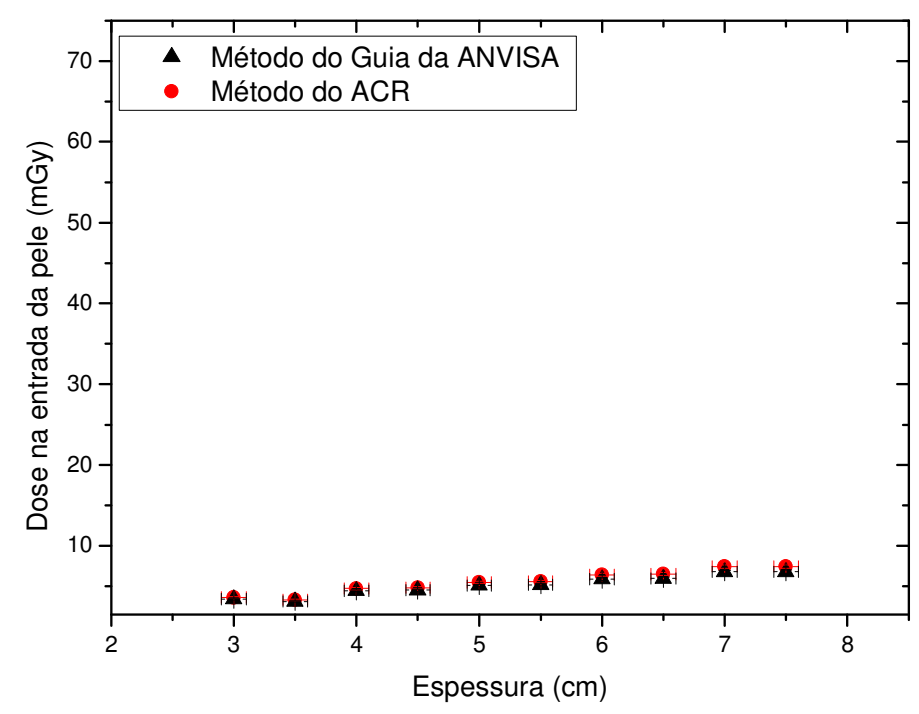

Figura 28 Comparação entre as estimativas de DEP calculada pelos dois métodos diferentes para cada espessura de mama comprimida estudada na projeção CC no equipamento GE DS - RhRh. 
Comparando os valores de DEP estimados pelos dois diferentes métodos, nota-se que os valores do método da ANVISA $^{13}$ são maiores que os estimados pelo método do $\mathrm{ACR}^{16}$ em $100 \%$ dos dados tanto na combinação Mo/Rh (Figuras 26 e 27) quanto na $\mathrm{Rh} / \mathrm{Rh}$ (Figuras 28 e 29) em ambas as projeções, porém esta diferença é sempre $\leq 1 \mathrm{mGy}$.

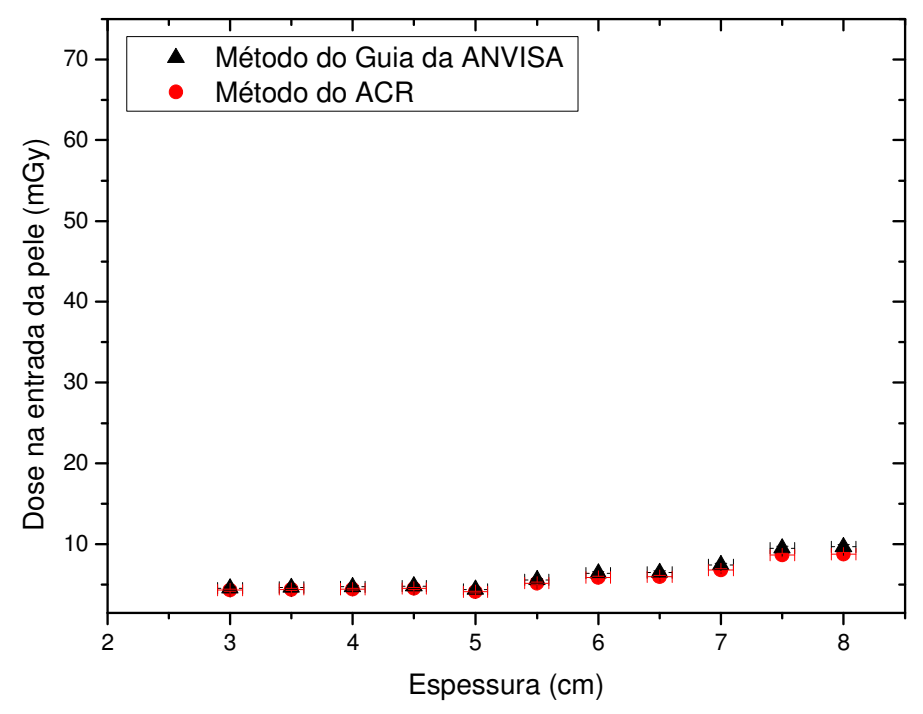

Figura 29 Comparação entre as estimativas de DEP calculada pelos dois métodos diferentes para cada espessura de mama comprimida estudada na projeção MLO no equipamento GE DS - RhRh.

\subsubsection{Comparação entre os três mamógrafos}

Comparando os valores de dose estimados pelo método da $\mathrm{ANVISA}^{13}$, na mesma combinação anodo-filtro Mo/Rh, nos dois diferentes equipamentos apresentados na Figura 30, projeção CC e Figura 31, projeção MLO se percebe que os valores de dose apresentados pelo equipamento Siemens são sempre maiores que os valores apresentados pelo equipamento GE DS. À medida que a espessura da mama comprimida aumenta, a diferença de dose também aumenta, alcançando 11 mGy na projeção CC e 19 mGy na projeção MLO.

Os valores de dose estimados pelo método do $\mathrm{ACR}^{16}$, na combinação anodofiltro Mo/Rh, obtidos no equipamento Siemens são sempre maiores que os valores estimados pelo equipamento GE DS em ambas as projeções (Figuras 32 e 33). Essa diferença também vai aumentando à medida que a espessura da 
mama aumenta chegando a 10 mGy na projeção CC (Figura 32) e 17 mGy na projeção MLO (Figura 33).

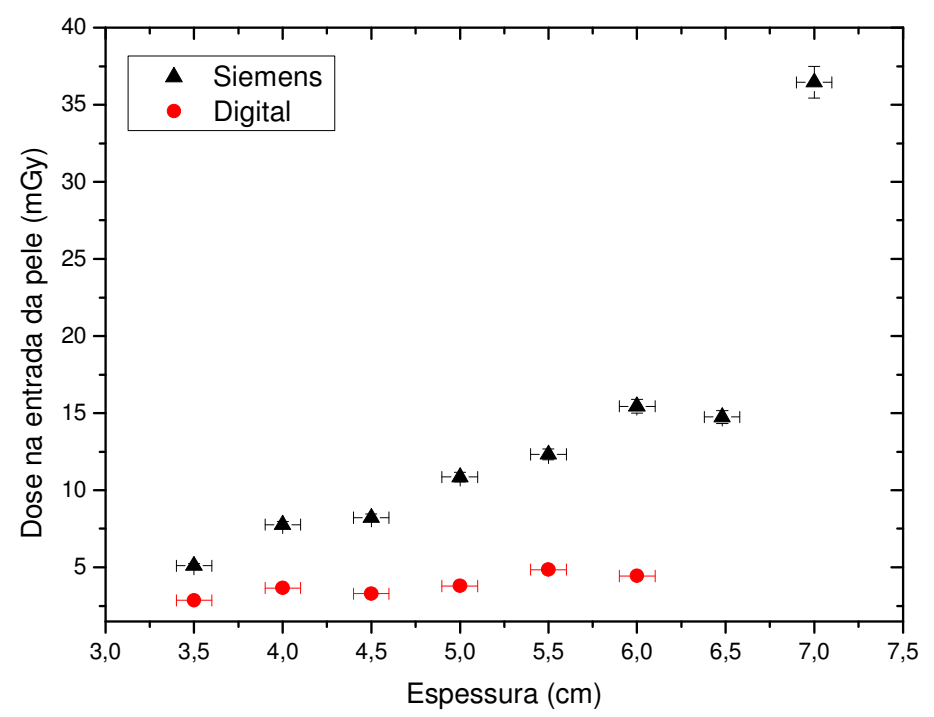

Figura 30 Comparação entre as estimativas de DEP calculada pelo método do Guia da ANVISA para cada espessura de mama comprimida estudada na projeção CC nos equipamentos Siemens e GE DS - MoRh.

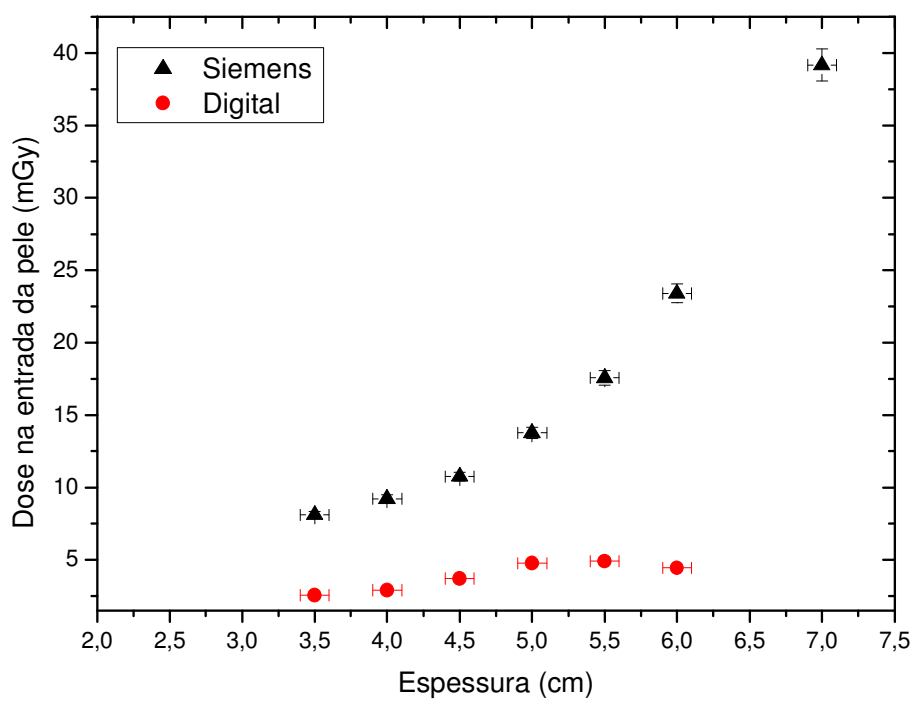

Figura 31 Comparação entre as estimativas de DEP calculada pelo método do Guia da ANVISA para cada espessura de mama comprimida estudada na projeção MLO nos equipamentos Siemens e GE DS - MoRh. 


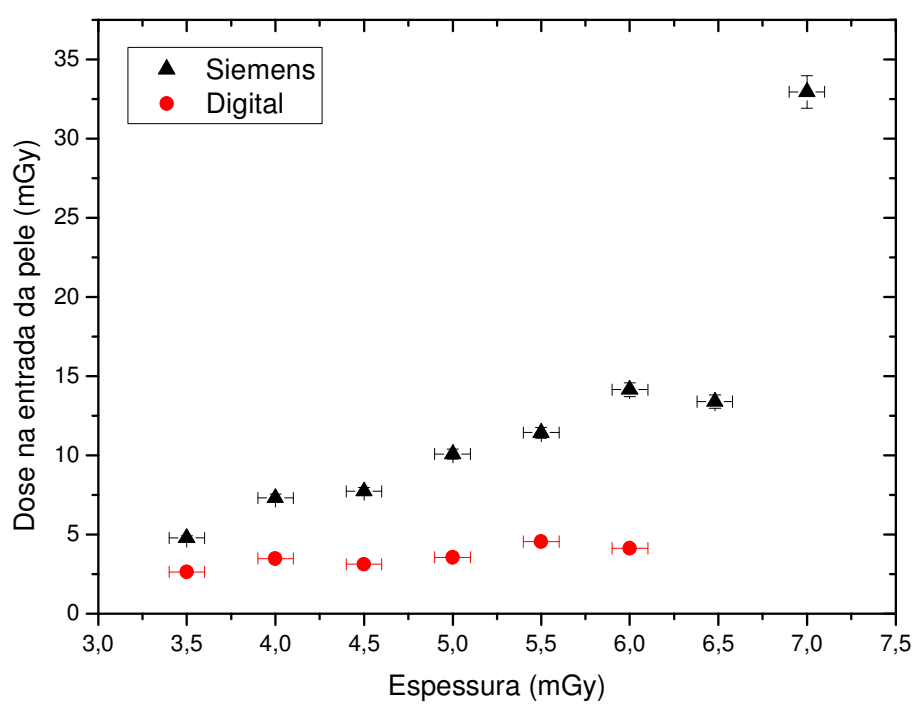

Figura 32 Comparação entre as estimativas de DEP calculada pelo método do Guia ACR para cada espessura de mama comprimida estudada na projeção CC nos equipamentos Siemens e GE DS - MoRh.

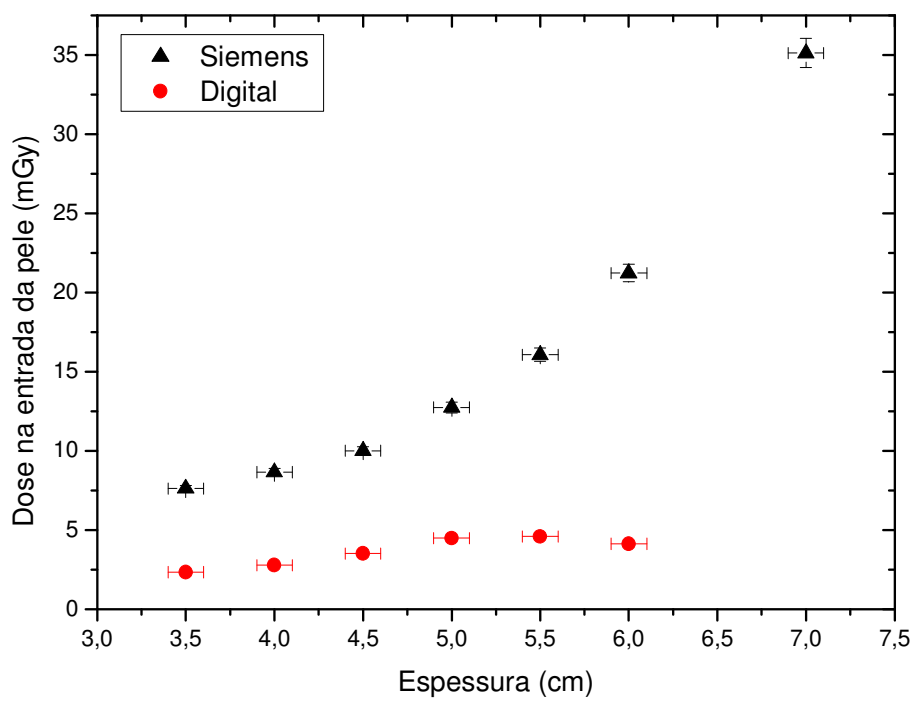

Figura 33 Comparação entre as estimativas de DEP calculada pelo método do Guia do ACR para cada espessura de mama comprimida estudada na projeção MLO nos equipamentos Siemens e GE DS - MoRh.

Ao comparar os valores de DEP obtidos pelo método do Guia da ANVISA ${ }^{13}$, nos equipamentos Siemens e GE 700 T, na combinação anodo-filtro Mo/Mo se observa que os valores, tanto na projeção CC (Figura 34), quanto na projeção MLO (Figura 35), são maiores no equipamento Siemens do que no GE DS. Na 
projeção CC a diferença de dose entre os valores não ultrapassa 3,5 mGy, com exceção da espessura $7,5 \mathrm{~cm}$, que esta diferença chega a aproximadamente 19 mGy. Na projeção MLO a diferença entre os valores são maiores e varia de 2,5 a 24,5 mGy.

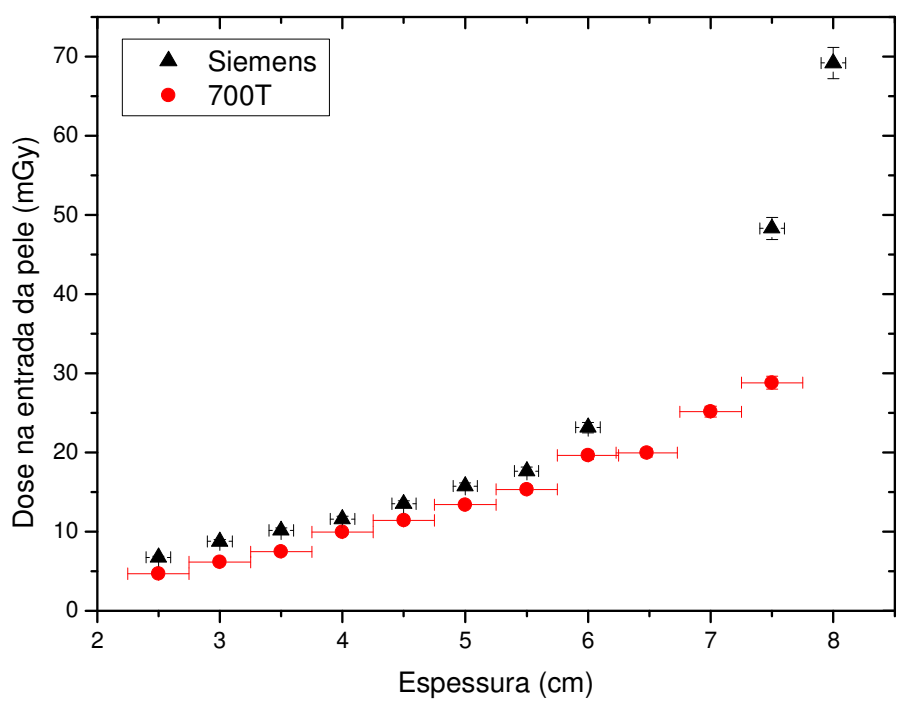

Figura 34 Comparação entre as estimativas de DEP calculada pelo método do Guia da ANVISA para cada espessura de mama comprimida estudada na projeção CC nos equipamentos Siemens e GE 700 T - MoMo.

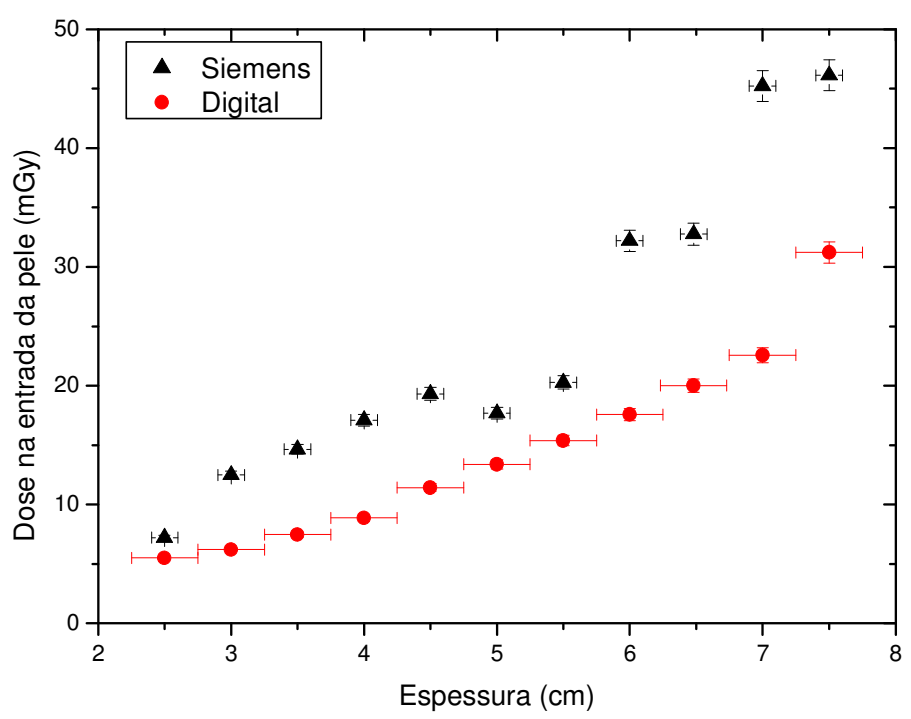

Figura 35 Comparação entre as estimativas de DEP calculada pelo método do Guia da ANVISA para cada espessura de mama comprimida estudada na projeção MLO nos equipamentos Siemens e GE 700 T - MoMo. 


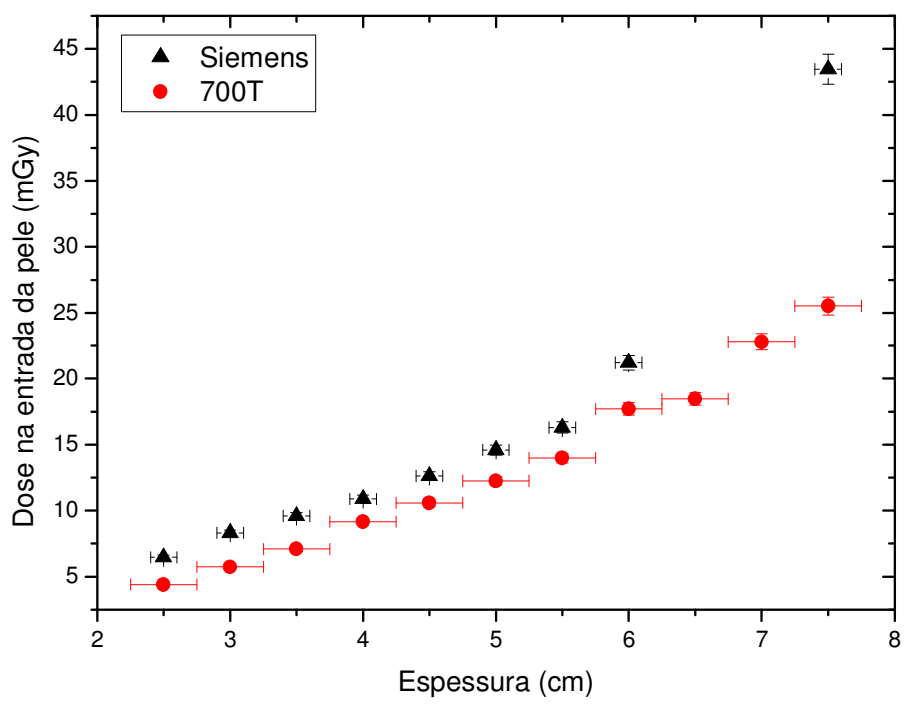

Figura 36 Comparação entre as estimativas de DEP calculada pelo método do Guia do ACR para cada espessura de mama comprimida estudada na projeção CC nos equipamentos Siemens e GE 700 T - MoMo.

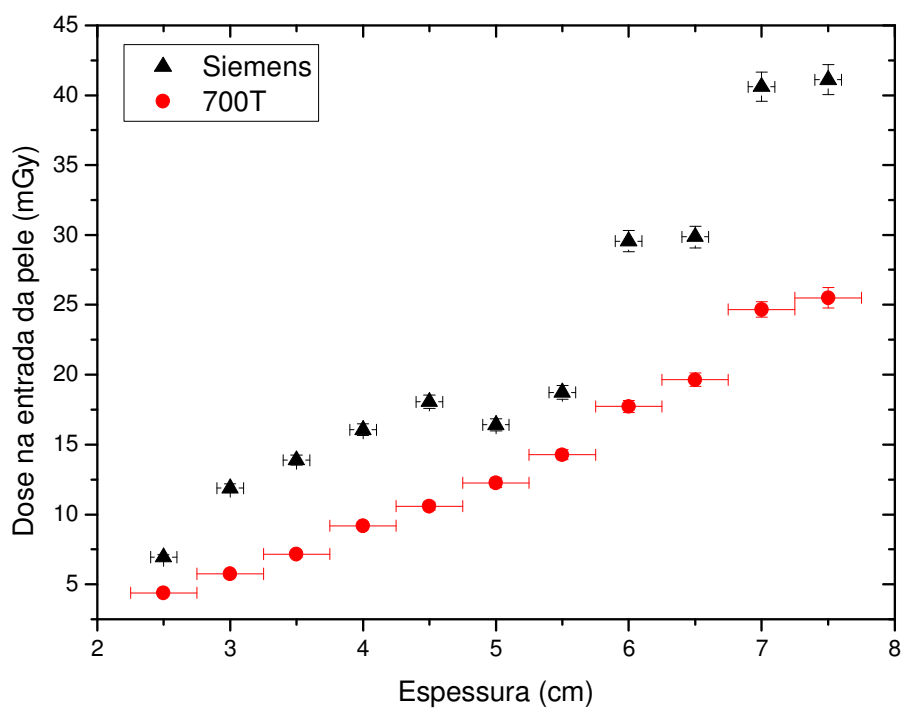

Figura 37 Comparação entre as estimativas de DEP calculada pelo método do Guia do ACR para cada espessura de mama comprimida estudada na projeção MLO nos equipamentos Siemens e GE 700 T - MoMo.

Os valores de DEP estimados pelo Guia do $A C R^{16}$, na combinação anodo-filtro Mo/Mo, também são maiores quando obtidos pelo equipamento Siemens em relação ao equipamento GE $700 \mathrm{~T}$ em ambas as projeções (Figuras 36 e 37). Na 
projeção CC (Figura 36) a diferença é menor do que na projeção MLO (Figura 37) e não passa de 3,5 mGy, com exceção da DEP na espessura $7,5 \mathrm{~cm}$, onde a diferença atinge $18 \mathrm{mGy}$. Na projeção MLO a diferença varia entre 2,5 a 16 mGy.

\subsubsection{Dose glandular média}

Baseando-se no método de Dance ${ }^{17,18}$, foi visto, na Tabela 2, que a glandularidade pode ser determinada para dois grupos etários das pacientes: mulheres com idade entre 40 e 49 anos e mulheres com idade entre 50 e 64 anos. Porém, neste estudo existem pacientes fora destes grupos e, portanto, efetuou-se um prolongamento para mulheres com idade inferior a 50 anos e mulheres com idade igual ou superior a 50 anos. A seguir, a Tabela 10 mostra a porcentagem de pacientes com idade abaixo de 40 anos ou acima de 64 anos para cada combinação anodo-filtro nos três mamógrafos estudados. Os resultados mostram que existe um número considerável de pacientes com idade fora da classificação apresentada na Tabela 2, porém como não existem valores tabelados para estes grupos, não houve outra maneira de estimar as doses para essas pacientes a não ser utilizando o método da extrapolação.

Tabela 10 Porcentagem de pacientes com idade abaixo de 40 anos e acima de 64 anos.

\begin{tabular}{|c|c|c|c|c|c|}
\hline \multirow{2}{*}{ Idade } & \multicolumn{5}{|c|}{ Porcentagem de pacientes (\%) } \\
\cline { 2 - 6 } & $\begin{array}{c}\text { GE 700T } \\
\text { Mo/Mo }\end{array}$ & $\begin{array}{c}\text { Siemens } \\
\text { Mo/Mo }\end{array}$ & $\begin{array}{c}\text { Siemens } \\
\text { Mo/Rh }\end{array}$ & $\begin{array}{c}\text { GE DS } \\
\text { Mo/Rh }\end{array}$ & $\begin{array}{c}\text { GE DS } \\
\text { Rh/Rh }\end{array}$ \\
\hline Abaixo de 40 anos & 6 & 11 & 0 & 23 & 11 \\
\hline Acima de 64 anos & 26 & 27 & 11 & 20 & 12 \\
\hline
\end{tabular}

Uma vez que o método do TRS $457^{22}$ se baseia no método de Dance ${ }^{17,18}$, esta consideração também é válida para ele.

\subsubsection{Método de Dance}

As Figuras 38 e 39 ilustram os valores estimados de dose glandular para diferentes espessuras de mama comprimida nas duas projeções estudadas, na 
combinação anodo-filtro Mo/Mo no equipamento GE $700 \mathrm{~T}$ para mulheres com idade inferior a 50 anos (Figura 38) e igual ou superior a 50 anos (Figura 39).

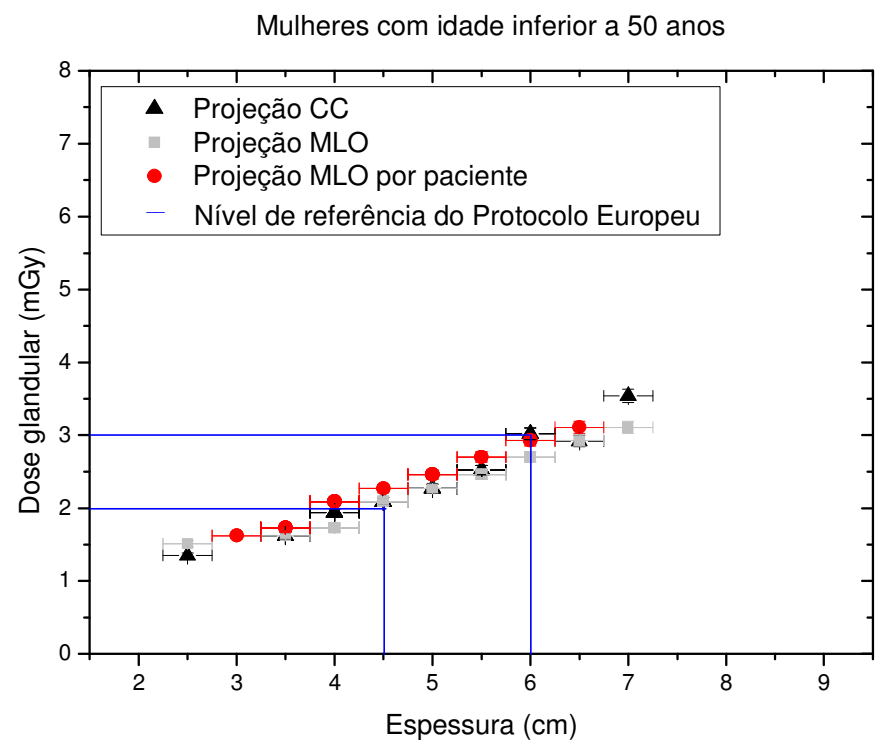

Figura 38 Dose glandular média para cada espessura de mama comprimida estudada na projeção CC e MLO no equipamento GE $700 \mathrm{~T}$, para mulheres com idade inferior a 50 anos.

Mulheres com idade igual ou superior a 50 anos

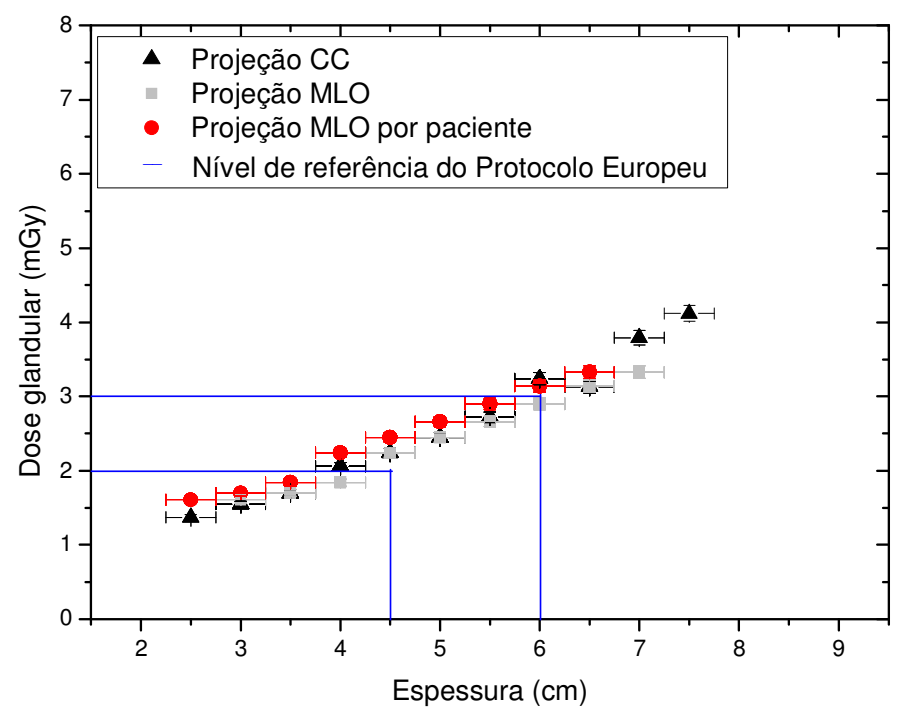

Figura 39 Dose glandular média para cada espessura de mama comprimida estudada na projeção CC e MLO no equipamento GE $700 \mathrm{~T}$, para mulheres com idade igual ou superior a 50 anos. 
As duas figuras também mostram que a dose recebida por uma mesma paciente durante a realização do exame na projeção MLO é sempre maior que na projeção CC, com exceção do resultado apresentado na espessura de $6 \mathrm{~cm}$, enquanto que para uma mesma espessura, a dose na projeção CC e MLO tem aproximadamente o mesmo comportamento.

O nível de referência de dose glandular determinado pelo Protocolo Europeu ${ }^{26}$ para uma espessura de 4,5 cm (padrão) é 2 mGy e para uma espessura de $6 \mathrm{~cm}$ é 3 mGy. Portanto, o resultado apresentado por este equipamento nas duas projeções está acima do nível de referência estipulado para a espessura menor e abaixo do nível de referência para a espessura maior.

Em todos os gráficos do equipamento Siemens (Figuras 40 a 43), observa-se que à medida que a espessura de mama comprimida aumenta, o valor de DGM também aumenta.

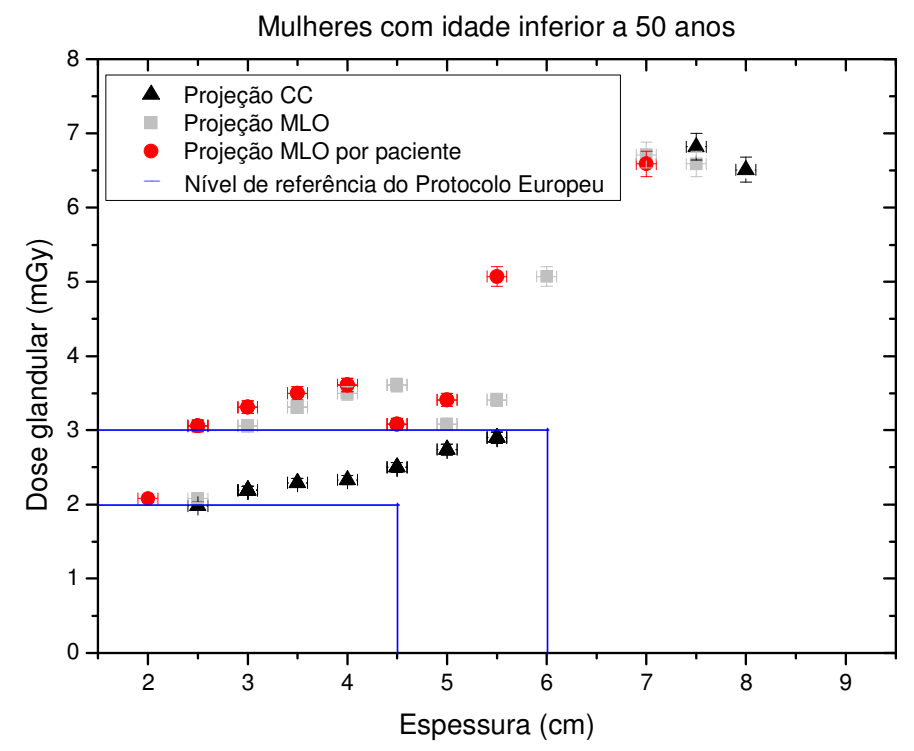

Figura 40 Dose glandular média para cada espessura de mama comprimida estudada na projeção CC e MLO no equipamento Siemens - Mo/Mo, para mulheres com idade inferior a 50 anos.

No equipamento Siemens, combinação Mo/Mo, em qualquer faixa etária, todos os valores de dose na projeção MLO (Figuras 40 e 41), tanto por espessura, quando por paciente, são maiores que os valores obtidos na projeção CC, com exceção da espessura $7,5 \mathrm{~cm}$ na faixa etária até 50 anos, em que o valor de dose 
na projeção CC é maior que o valor na projeção MLO. Em ambas as faixas etárias, os valores de dose estão acima do nível de referência.

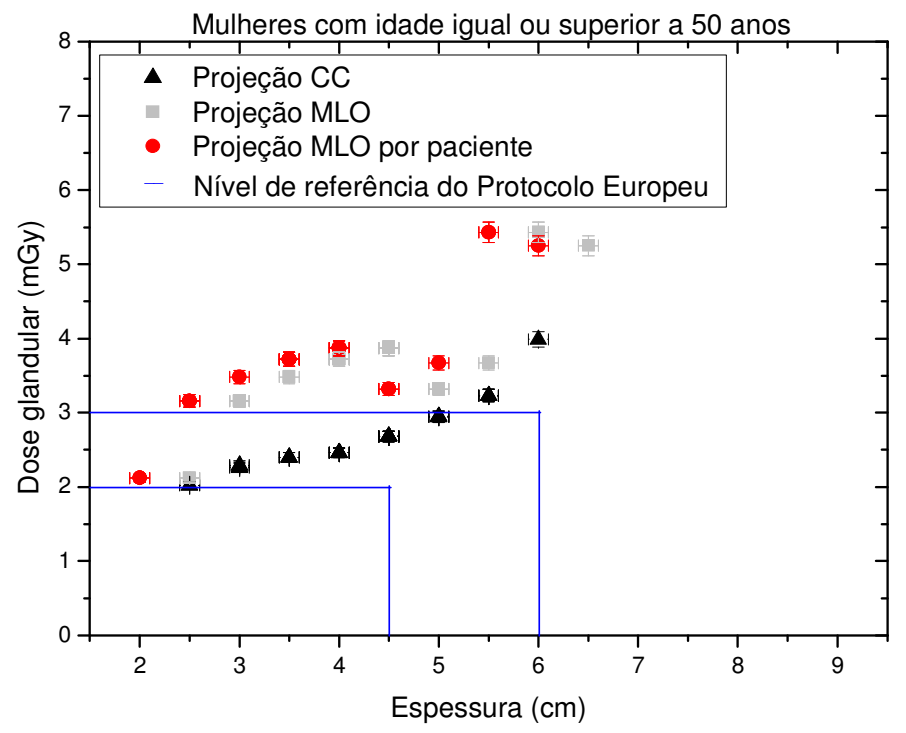

Figura 41 Dose glandular média para cada espessura de mama comprimida estudada na projeção CC e MLO no equipamento Siemens - Mo/Mo, para mulheres com idade igual ou superior a 50 anos.

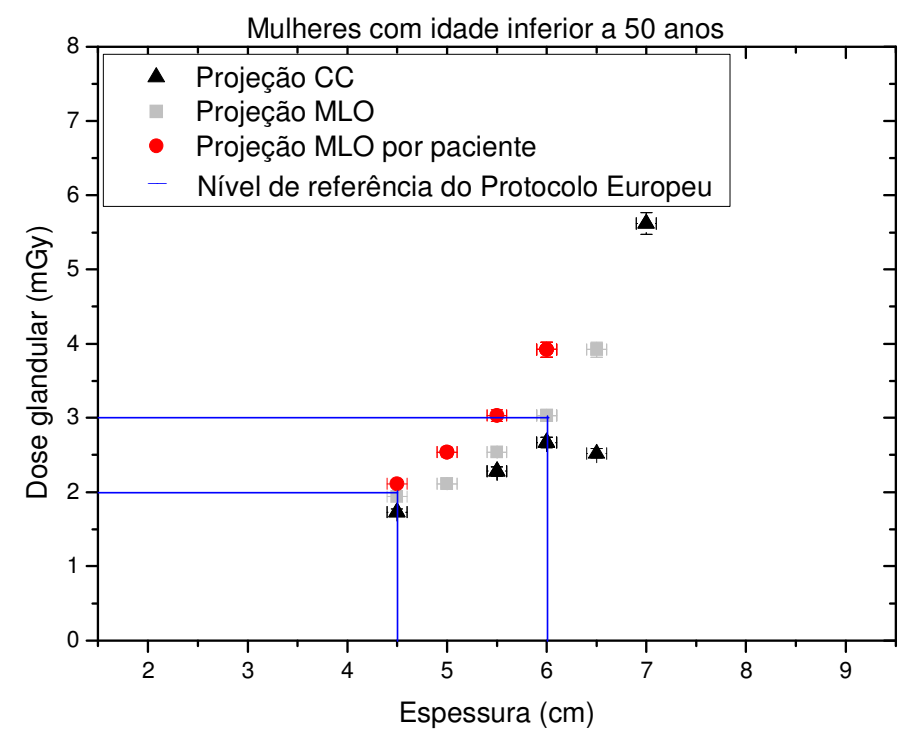

Figura 42 Dose glandular média para cada espessura de mama comprimida estudada na projeção CC e MLO no equipamento Siemens - Mo/Rh, para mulheres com idade inferior a 50 anos. 


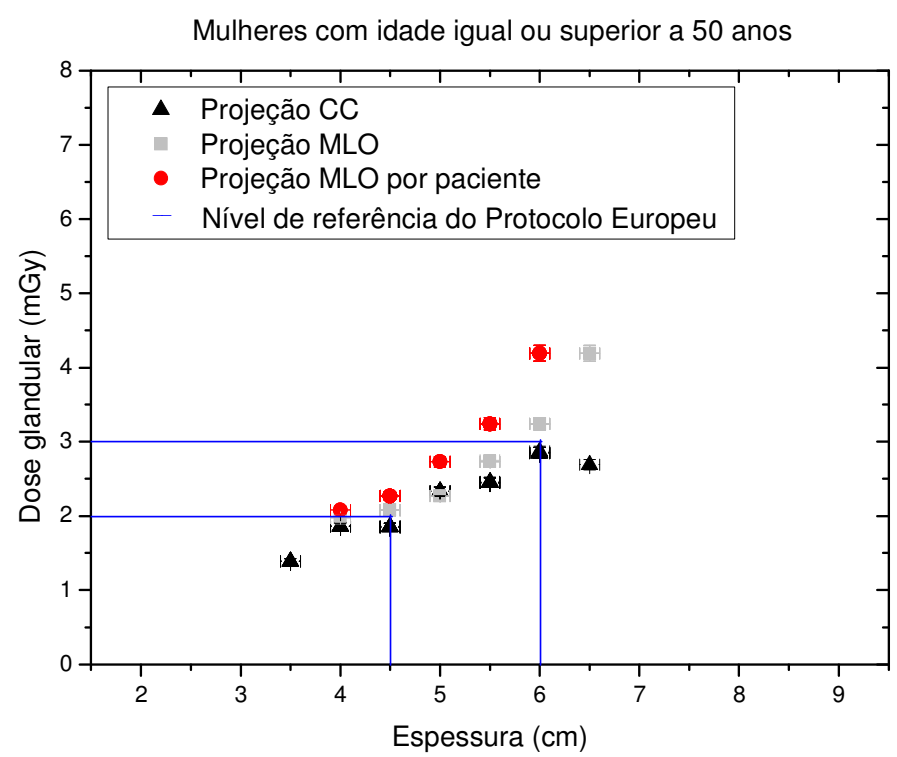

Figura 43 Dose glandular média para cada espessura de mama comprimida estudada na projeção CC e MLO no equipamento Siemens - Mo/Rh, para mulheres com idade igual ou superior a 50 anos.

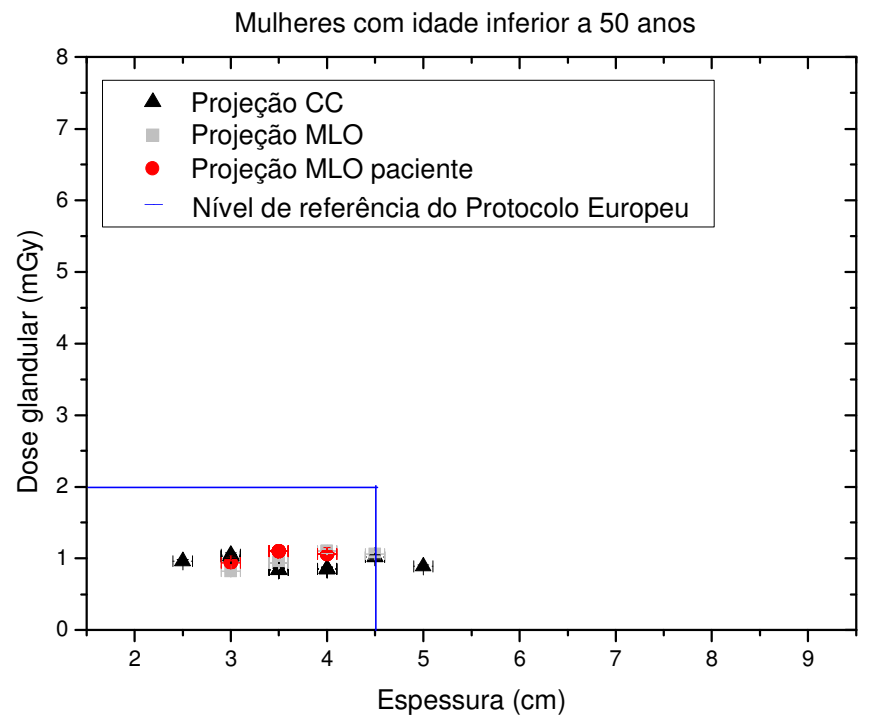

Figura 44 Dose glandular média para cada espessura de mama comprimida estudada na projeção CC e MLO no equipamento GE DS - Mo/Rh, para mulheres com idade inferior a 50 anos. 


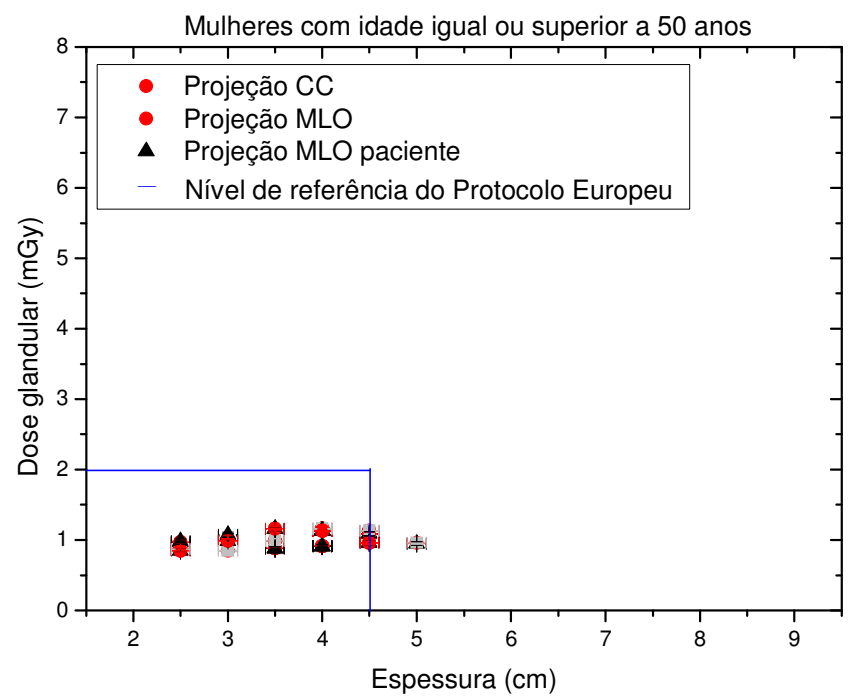

Figura 45 Dose glandular média para cada espessura de mama comprimida estudada na projeção CC e MLO no equipamento GE DS - Mo/Rh, para mulheres com idade igual ou superior a 50 anos.

$\mathrm{Na}$ combinação Mo/Rh (Figuras 42 e 43) todos os valores de dose apresentados na projeção MLO, tanto por espessura, quanto por paciente, estão acima dos valores apresentados na projeção CC, com exceção da espessura $5 \mathrm{~cm}$ na faixa etária maior ou igual a 50 anos, em que os valores são quase coincidentes.

Todos os valores de dose apresentados na projeção CC estão abaixo do nível de referência para ambas as faixas etárias (Figuras 42 e 43), enquanto que os valores de dose da projeção MLO, apenas um valor está abaixo do nível de referência, que é valor correspondente à espessura $4,5 \mathrm{~cm}$ para mulheres com idade inferior a 50 anos.

Os valores de dose apresentados pelo equipamento GE DS são praticamente coincidentes, a variação entre eles é menor que 0,3 mGy para a combinação anodo-filtro Mo/Rh (Figuras 44 e 45) e menor que 0,7 mGy para Rh/Rh (Figuras 46 e 47). Todos os valores de DGM estão abaixo do nível de referência. 


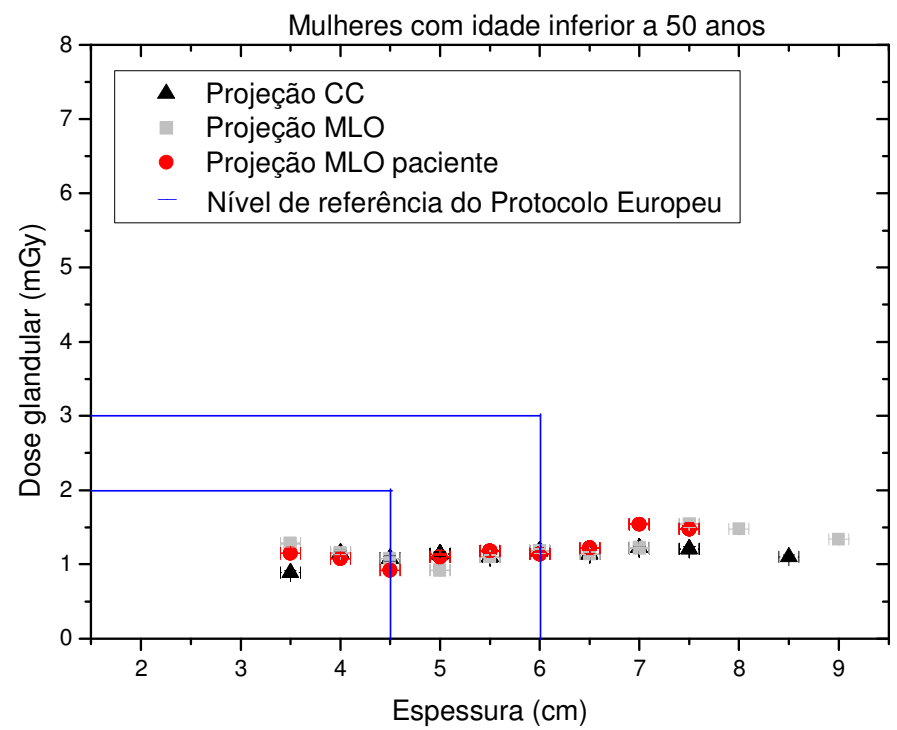

Figura 46 Dose glandular média para cada espessura de mama comprimida estudada na projeção CC e MLO no equipamento GE DS - Rh/Rh, para mulheres com idade inferior a 50 anos.

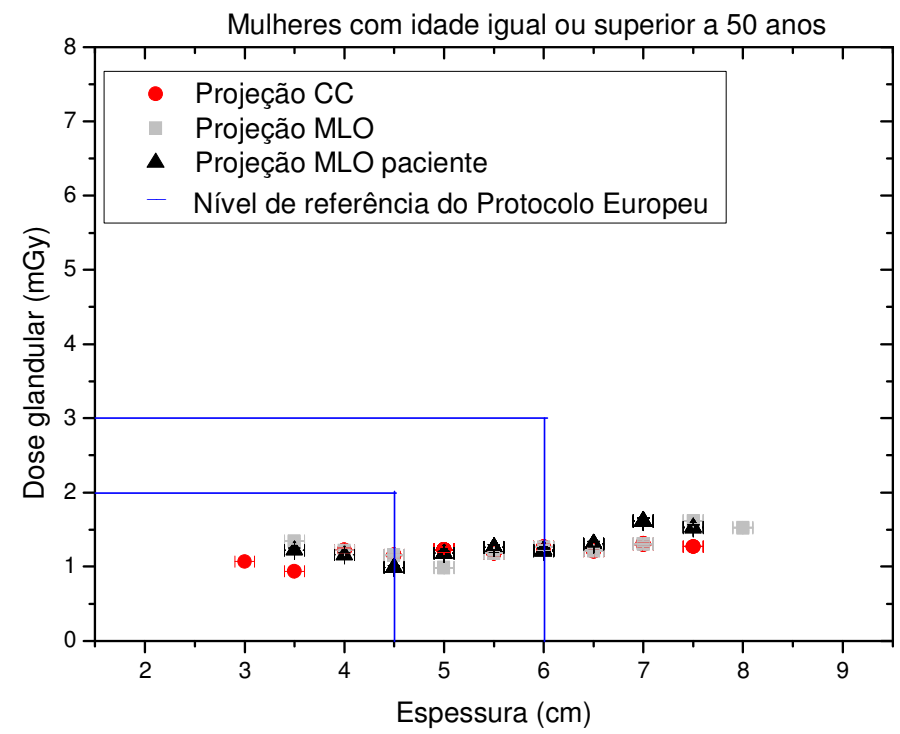

Figura 47 Dose glandular média para cada espessura de mama comprimida estudada na projeção CC e MLO no equipamento GE DS - Rh/Rh, para mulheres com idade igual ou superior a 50 anos. 


\subsubsection{Método de Wu}

A Figura 48 compara a dose glandular média recebida em cada projeção durante o exame de mamografia realizado no equipamento GE $700 \mathrm{~T}$ utilizando a combinação de anodo-filtro Mo/Mo.

Nesse gráfico também se constata que não há grandes diferenças entre as doses recebidas para uma mesma espessura entre as duas projeções. Nota-se diferença ao analisar a dose glandular recebida por uma mesma paciente nas duas projeções, que é sempre maior na projeção MLO.

O nível de referência de dose glandular determinado pelo $A C R^{16}$ para uma espessura de 4,2 cm (padrão) é $3 \mathrm{mGy}$. Portanto, o resultado apresentado por este equipamento nas duas projeções está abaixo do nível de referência.

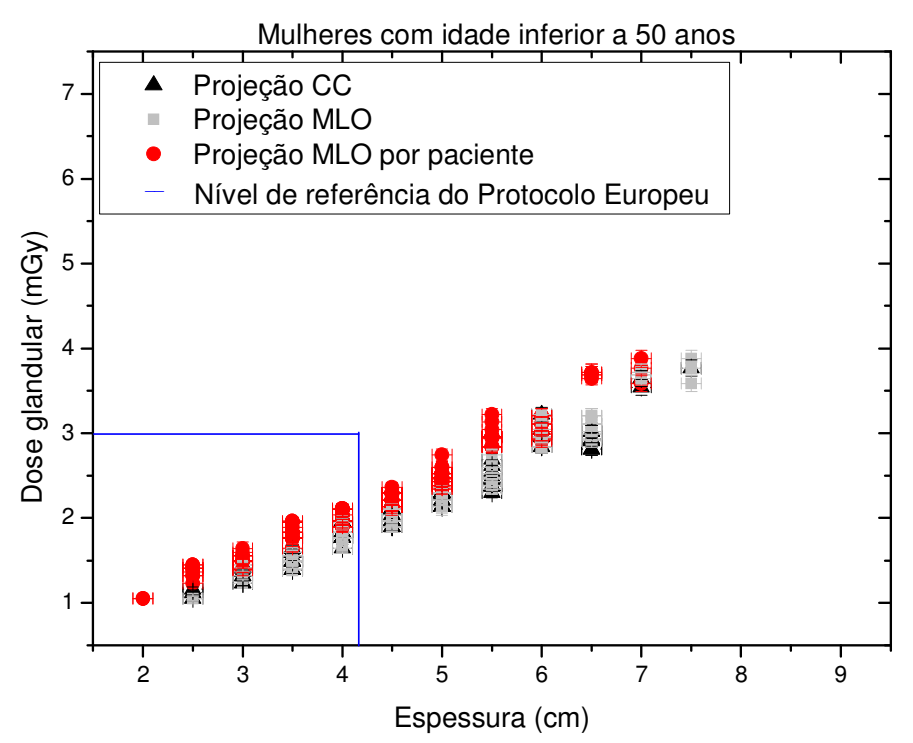

Figura 48 Dose glandular média para cada espessura de mama comprimida estudada na projeção CC e MLO no equipamento GE $700 \mathrm{~T}$.

No equipamento Siemens, em ambas as combinações anodo-filtro (Figuras 49 e 50), nota-se que todos os valores de DGM na projeção CC são menores que os valores da projeção MLO, tanto por espessura, quanto por paciente, com exceção de um valor na espessura $7,5 \mathrm{~cm}$, na combinação anodo-filtro Mo/Mo (Figura 49), em que os valores são praticamente coincidentes. Em ambas as projeções, os valores de dose obtidos na projeção CC estão abaixo do nível de referência, enquanto que os valores estimados na projeção MLO estão acima. 


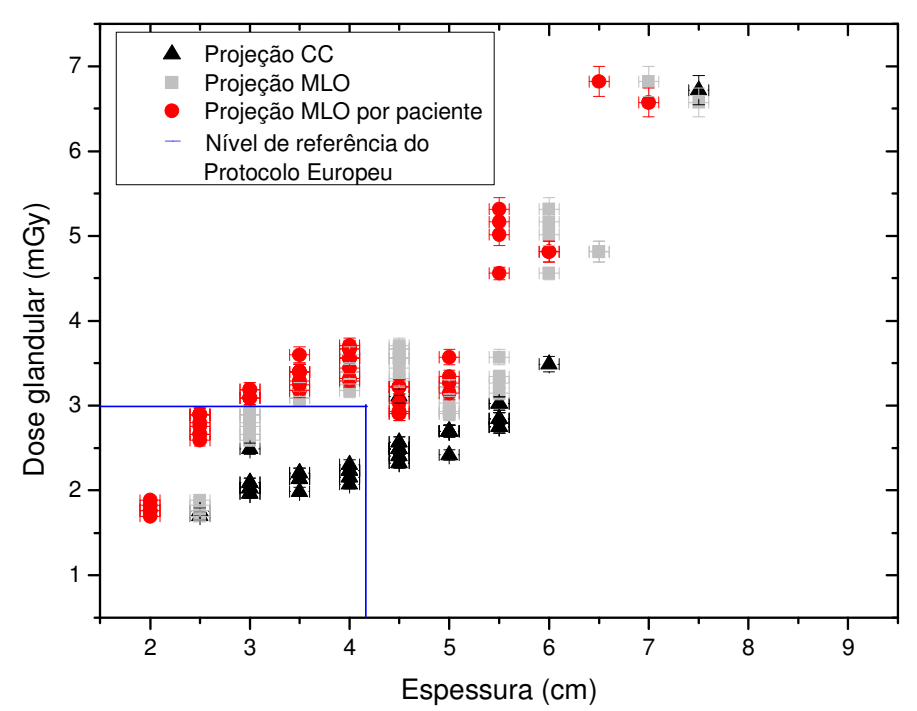

Figura 49 Dose glandular média para cada espessura de mama comprimida estudada na projeção CC e MLO no equipamento Siemens - Mo/Mo.

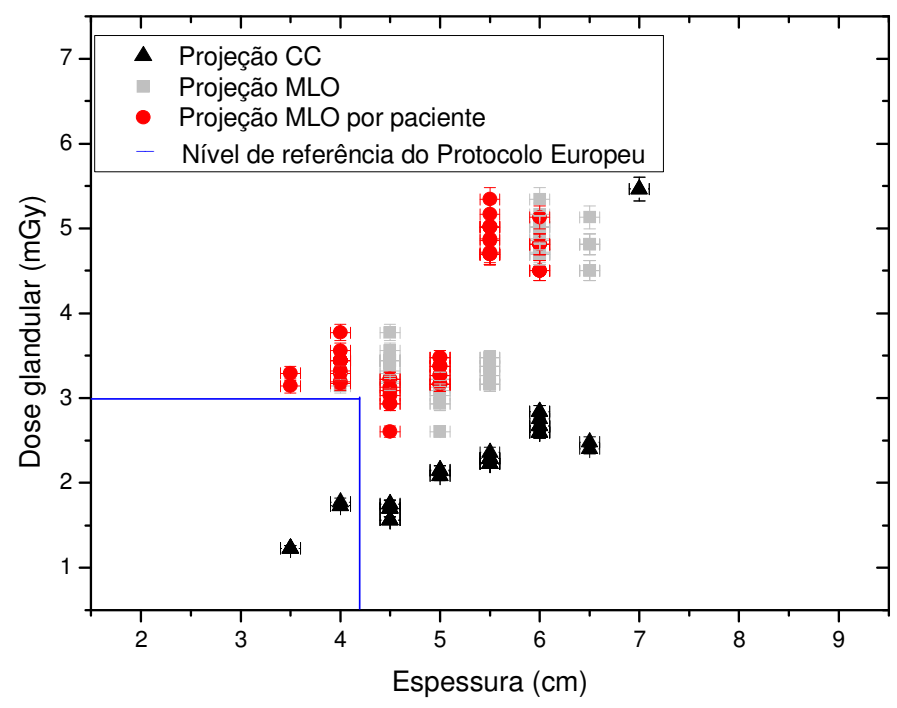

Figura 50 Dose glandular média para cada espessura de mama comprimida estudada na projeção CC e MLO no equipamento Siemens - Mo/Rh.

No equipamento GE DS (Figuras 51 e 52), os valores de dose são praticamente coincidentes, havendo uma diferença menor que 0,35 mGy em ambas as combinações anodo-filtro. As doses estimadas estão abaixo da metade do valor do nível de referência. 


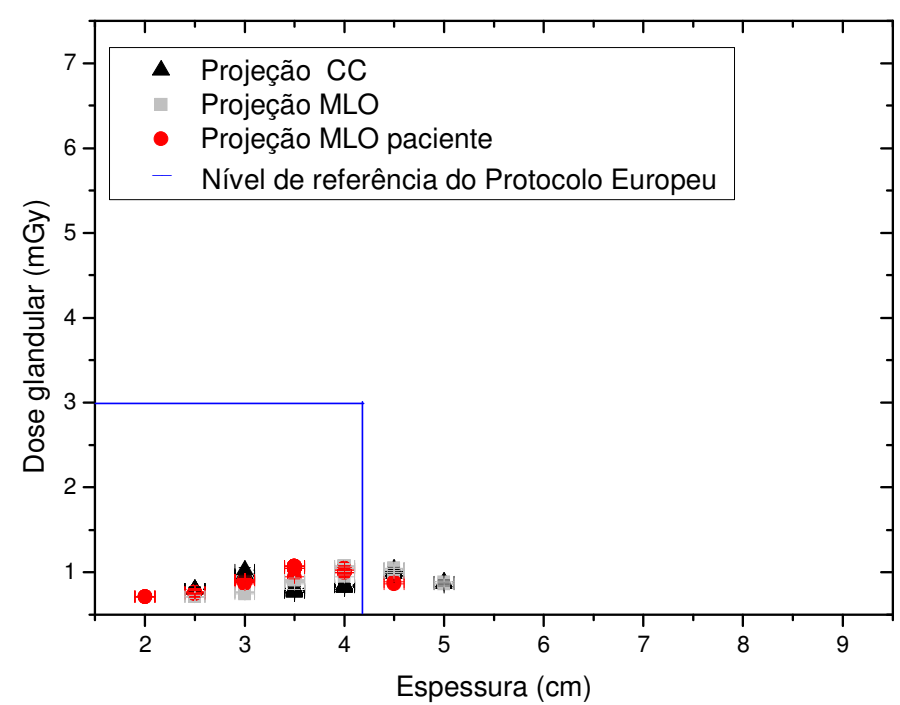

Figura 51 Dose glandular média para cada espessura de mama comprimida estudada na projeção CC e MLO no equipamento GE DS - Mo/Rh.

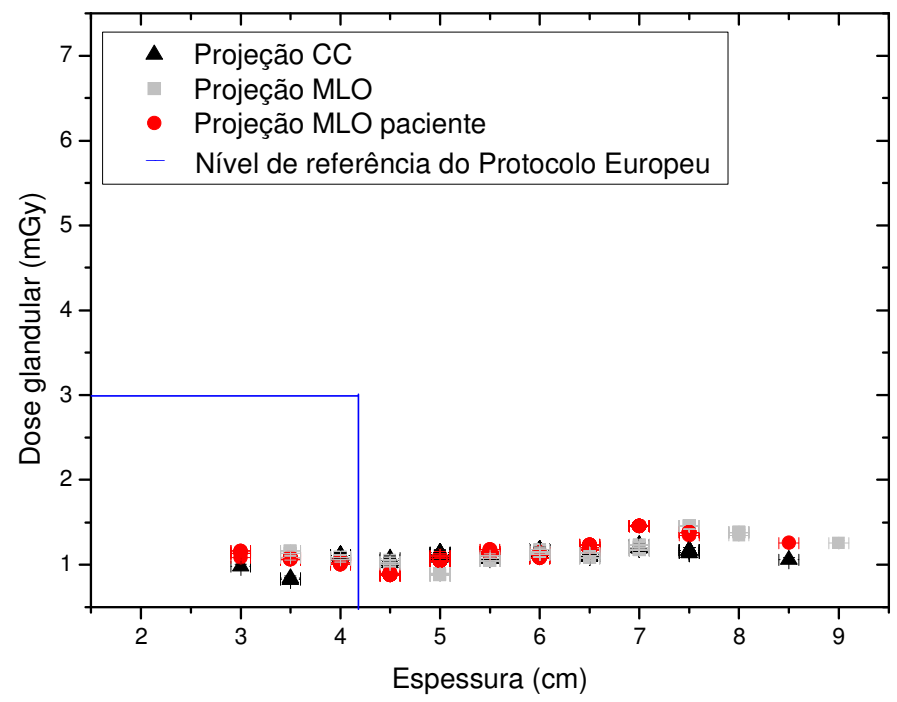

Figura 52 Dose glandular média para cada espessura de mama comprimida estudada na projeção CC e MLO no equipamento GE DS - Rh/Rh. 


\subsubsection{Método do TRS 457}

As Figuras 53 a 62 ilustram a comparação de dose glandular estimada por meio deste método entre as duas projeções para os dois grupos de idades das mulheres.

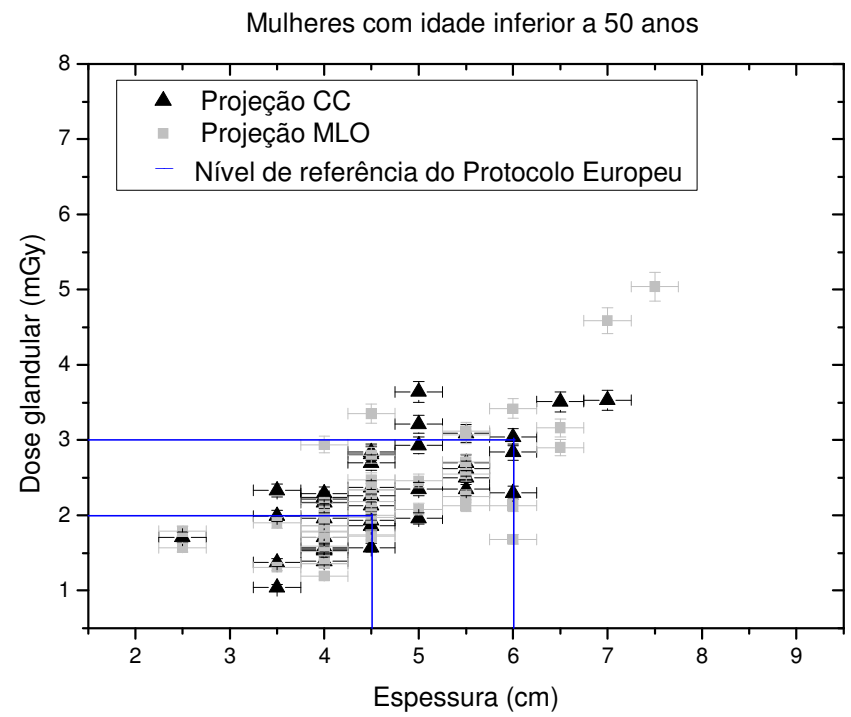

Figura 53 Dose glandular média para cada espessura de mama comprimida estudada na projeção CC e MLO no equipamento GE $700 \mathrm{~T}$, para mulheres com idade inferior a 50 anos.

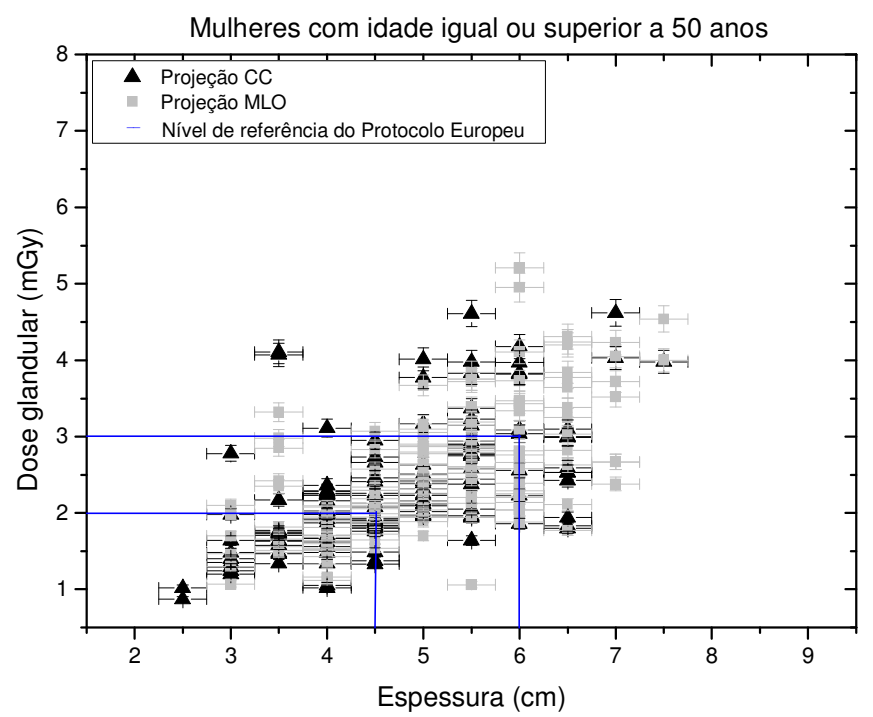

Figura 54 Dose glandular média para cada espessura de mama comprimida estudada na projeção CC e MLO no equipamento GE 700 T, para mulheres com idade igual ou superior a 50 anos. 
No equipamento GE 700 T (Figuras 53 e 54), os valores de DGM, para os dois grupos de idade das pacientes, para uma mesma espessura, tanto na projeção CC quanto na MLO, variam bastante. Para o grupo das mulheres com idade inferior a 50 anos (Figura 53), em ambas as projeções, os valores de DGM, variam até 2 mGy para uma mesma espessura. Enquanto que no outro grupo (Figura 54) essa variação chega a $3 \mathrm{mGy}$. Isso ocorre, pois neste método é possível estimar a DGM que cada paciente recebeu durante o exame.

Em virtude desta grande variação, não é válido comparar a DGM recebida por uma mesma paciente e por uma mesma espessura na projeção MLO.

O nível de referência de dose glandular determinado pelo Protocolo Europeu ${ }^{26}$ para uma espessura de $4,5 \mathrm{~cm}$ (padrão) é 2 mGy e para uma espessura de $6 \mathrm{~cm}$ é 3 mGy. Portanto, o resultado apresentado por este equipamento, para o grupo das mulheres com idade inferior a 50 anos (Figura 53) e igual ou superior a 50 anos (Figura 54), nas duas projeções, está acima do nível de referência estipulado para $4,5 \mathrm{~cm}$ em $63 \%$ e em $51 \%$ das espessuras estudadas, respectivamente. $O$ nível de referência para a espessura de $6 \mathrm{~cm}$ é atendido em $49 \%$ das espessuras estudadas no primeiro grupo citado e em $63 \%$ no segundo grupo citado.

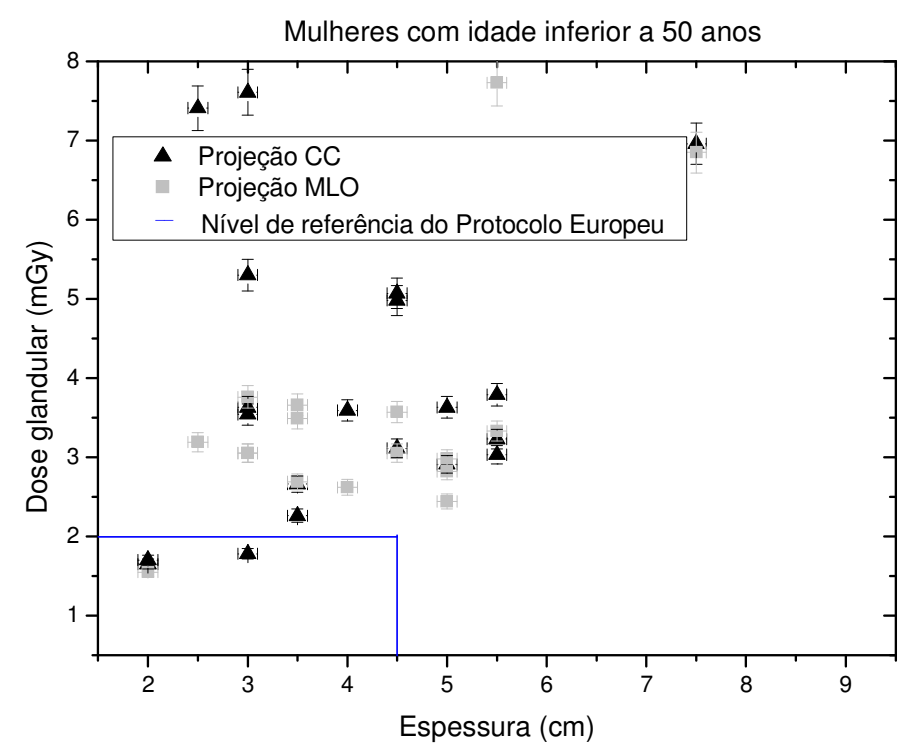

Figura 55 Dose glandular média para cada espessura de mama comprimida estudada na projeção CC e MLO no equipamento Siemens - Mo/Mo, para mulheres com idade inferior a 50 anos. 


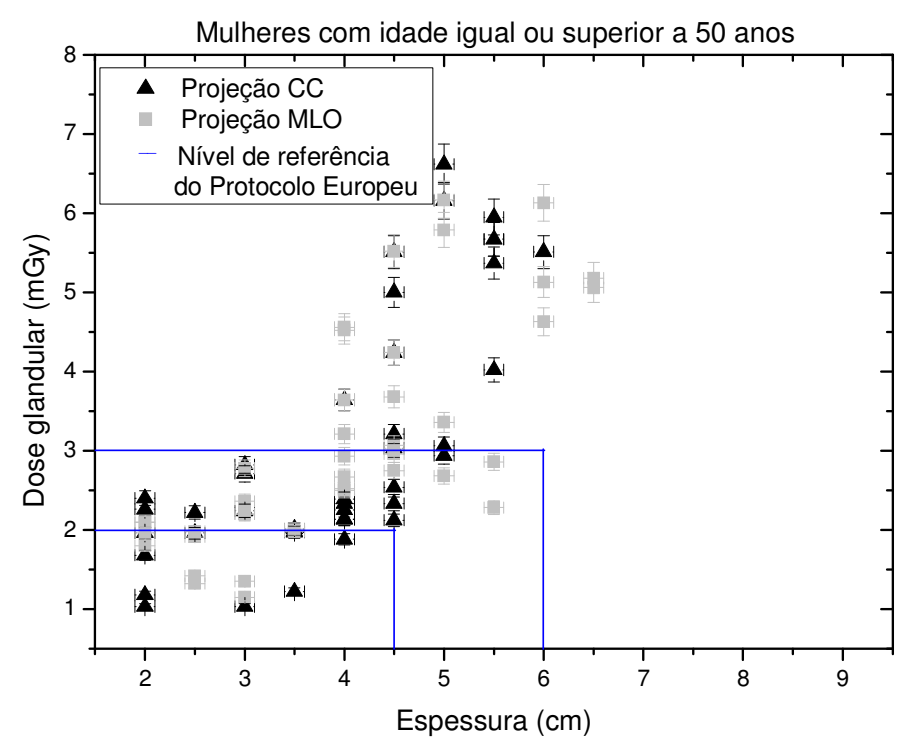

Figura 56 Dose glandular média para cada espessura de mama comprimida estudada na projeção CC e MLO no equipamento Siemens - Mo/Mo, para mulheres com idade igual ou superior a 50 anos.

No equipamento Siemens, na combinação anodo-filtro Mo/Mo a variação de dose, para uma mesma espessura, é ainda maior, chega a 6 mGy na projeção CC e 4,5 mGy na projeção MLO para mulheres com idade inferior a 50 anos (Figura 55). No outro grupo com faixa etária maior (Figura 56), a diferença chega a 4 mGy em ambas as projeções. Todos os valores de dose estimados para a espessura de $4,5 \mathrm{~cm}$ estão acima do nível de referência.

$\mathrm{Na}$ combinação anodo-filtro $\mathrm{Mo} / \mathrm{Rh}$, como existem poucos dados na faixa etária menor que 50 anos (Figura 57), a diferença entre os valores também é menor. Na projeção CC, a diferença, para uma mesma espessura, é menor que 1,5 mGy e para a projeção MLO, menor que 2,5 mGy. Os valores de dose estimados para a espessura de $4,5 \mathrm{~cm}$ está abaixo do nível de referência e para $6 \mathrm{~cm}$, na projeção CC está acima ou igual e na MLO abaixo. Para o grupo das mulheres com idade maior ou igual a 50 anos (Figura 58), os valores de dose, para uma mesma espessura variam 3,5 mGy na projeção CC e 2,5 mGy na projeção MLO. Na espessura 4,5 cm, metade dos valores da projeção CC e $70 \%$ dos valores estimados na MLO estão acima do nível de referência. No caso da espessura de $6 \mathrm{~cm}$, esses valores mudam para $78 \%$ e $45 \%$ respectivamente. 


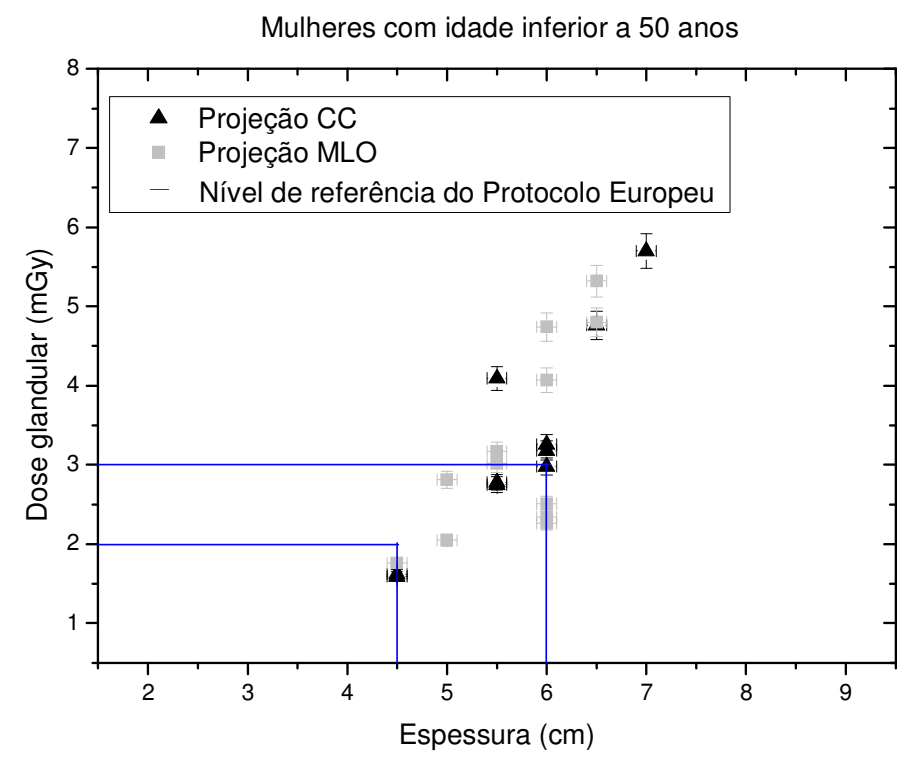

Figura 57 Dose glandular média para cada espessura de mama comprimida estudada na projeção CC e MLO no equipamento Siemens - Mo/Rh, para mulheres com idade inferior a 50 anos.

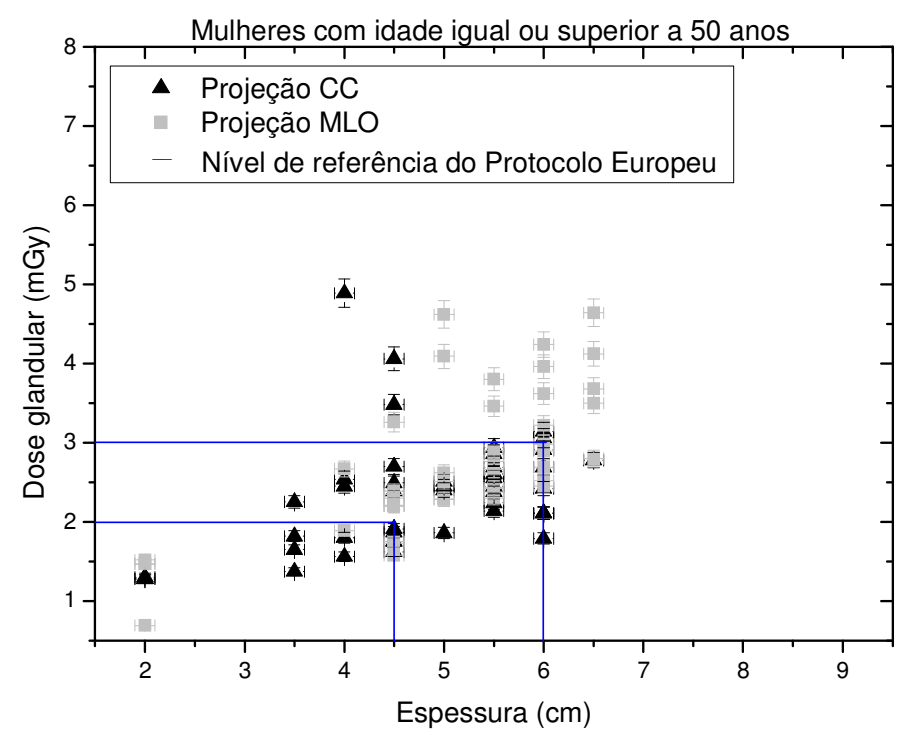

Figura 58 Dose glandular média para cada espessura de mama comprimida estudada na projeção CC e MLO no equipamento Siemens - Mo/Rh, para mulheres com idade igual ou superior a 50 anos.

Os valores de DGM obtidos no equipamento GE DS, para uma mesma espessura, são bastante próximos para mulheres com qualquer idade, em ambas as combinações anodo-filtro (Figuras 59, 60, 61 e 62), pois esta diferença não 
passa de 1 mGy. Todos os valores estão abaixo do nível de referência apresentado pelo Protocolo Europeu ${ }^{26}$.

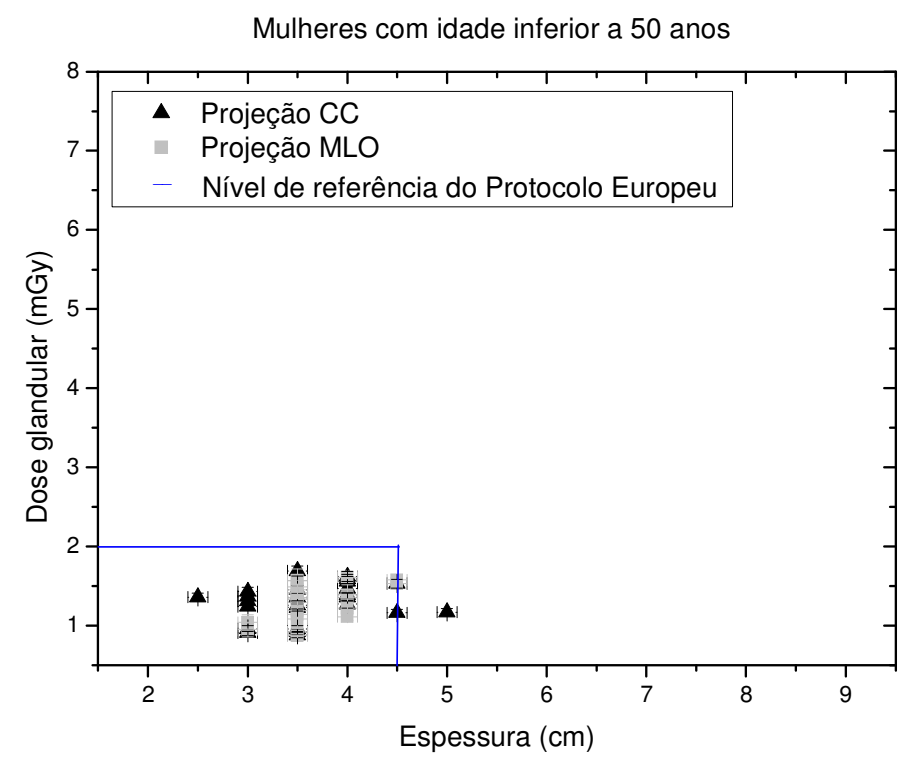

Figura 59 Dose glandular média para cada espessura de mama comprimida estudada na projeção CC e MLO no equipamento GE DS - Mo/Rh, para mulheres com idade inferior a 50 anos.

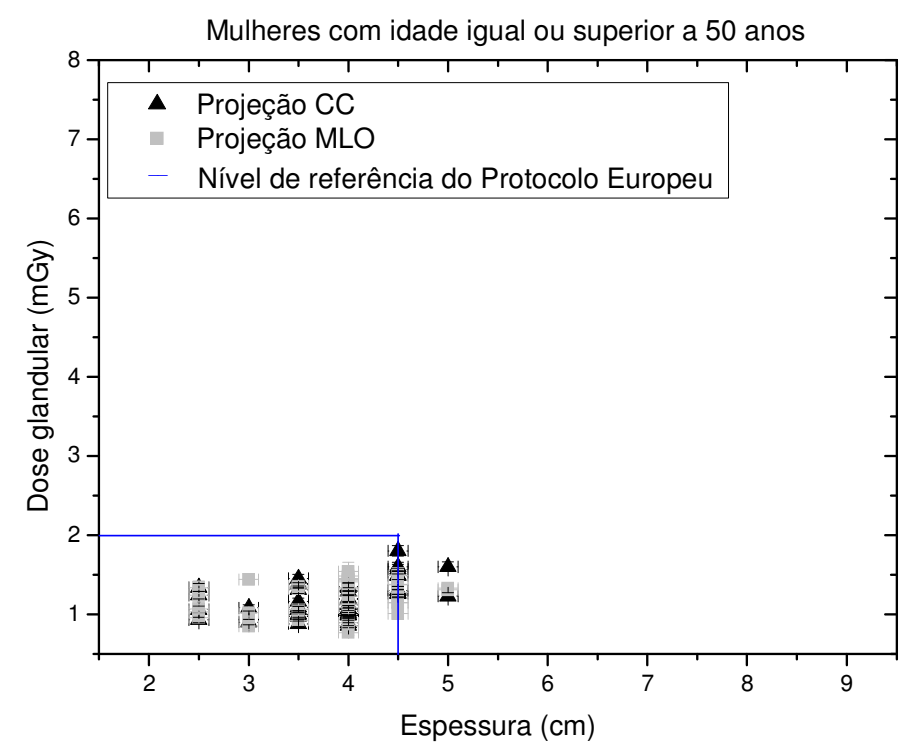

Figura 60 Dose glandular média para cada espessura de mama comprimida estudada na projeção CC e MLO no equipamento GE DS - Mo/Rh, para mulheres com idade igual ou superior a 50 anos. 




Figura 61 Dose glandular média para cada espessura de mama comprimida estudada na projeção CC e MLO no equipamento GE DS - Rh/Rh, para mulheres com idade inferior a 50 anos.

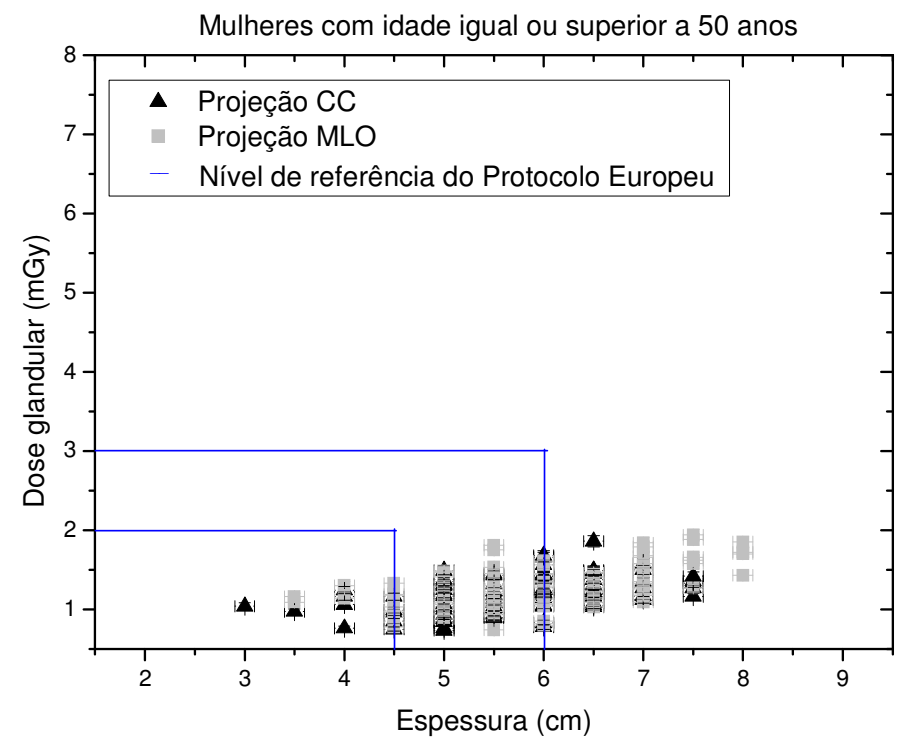

Figura 62 Dose glandular média para cada espessura de mama comprimida estudada na projeção CC e MLO no equipamento GE DS - Rh/Rh, para mulheres com idade igual ou superior a 50 anos. 


\subsubsection{Comparação entre os três métodos}

A comparação entre os três métodos pode ser visualizada para mulheres com idade inferior a 50 anos, na projeção CC, por meio das Figuras 63, 67, 71, 75 e 79 e na projeção MLO nas Figuras 64, 68, 72, 76 e 80, para os equipamentos e combinações GE 700 T - Mo/Mo, Siemens - Mo/Mo, Siemens - Mo/Rh, GE DS Mo/Rh e GE DS - Rh/Rh, respectivamente. Para esses mesmos equipamentos e combinações anodo-filtro, para mulheres com idade igual ou superior a 50 anos compara-se os resultados de dose glandular média entre os três métodos, na projeção CC, como mostram as Figuras $65,69,73,77$ e 81 e, na projeção MLO, as Figuras $66,70,74,78$ e 82.

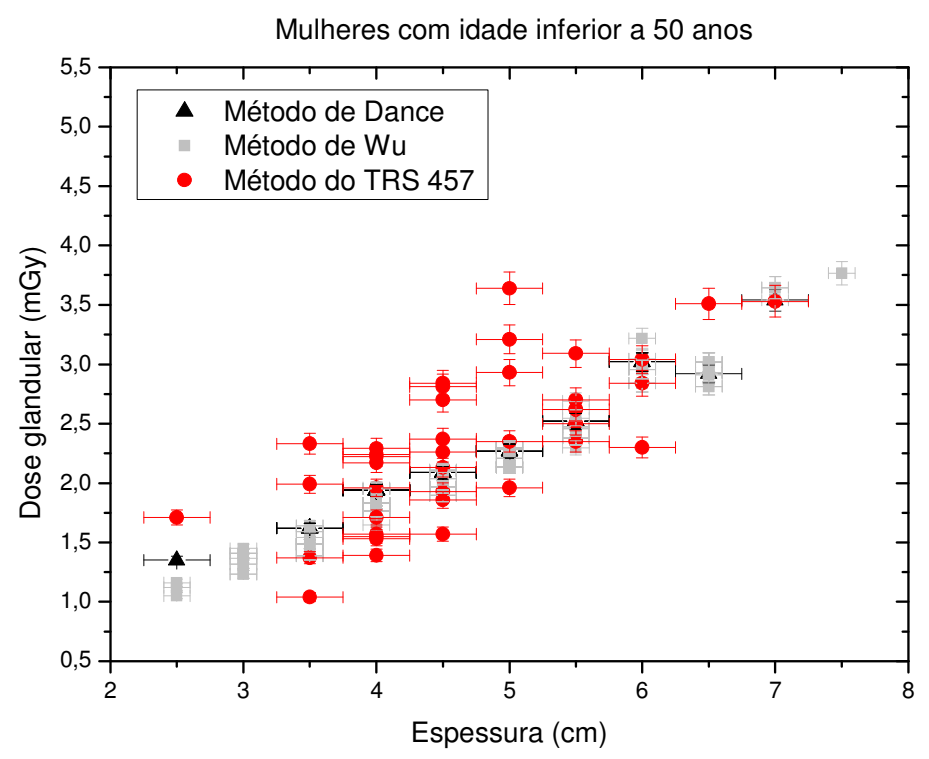

Figura 63 Comparação entre a estimativa de dose glandular média calculada pelos três métodos diferentes para cada espessura de mama comprimida estudada na projeção CC no equipamento GE 700 T, para mulheres com idade inferior a 50 anos.

O equipamento GE 700 T (Figuras 63 a 66) mostra resultados cujos valores de DGM estimados pelo método de Dance ${ }^{17,18}$ são maiores que os estimados pelo método de $\mathrm{Wu}^{19,20,21} \mathrm{em} 67 \%$ das espessuras estudadas.

Os valores de DGM calculados pelo método do TRS $457^{22}$ variam cerca de 2 mGy para o grupo de mulheres com idade inferior a 50 anos (Figuras 63 e 64) e cerca de 3 mGy para o grupo com idade igual ou superior a 50 anos (Figuras 65 e 
66). Porém todos os valores de DGM estimados por este método giram em torno dos valores estimados pelos outros dois métodos analisados.

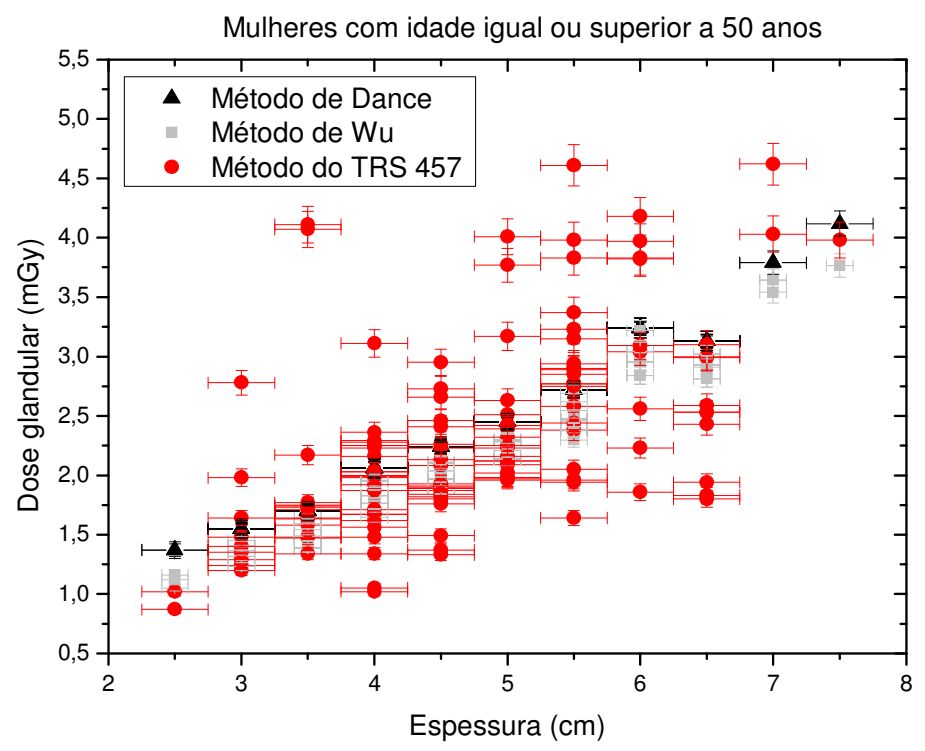

Figura 64 Comparação entre a estimativa de dose glandular média calculada pelos três métodos diferentes para cada espessura de mama comprimida estudada na projeção CC no equipamento GE $700 \mathrm{~T}$, para mulheres com idade igual ou superior a 50 anos.

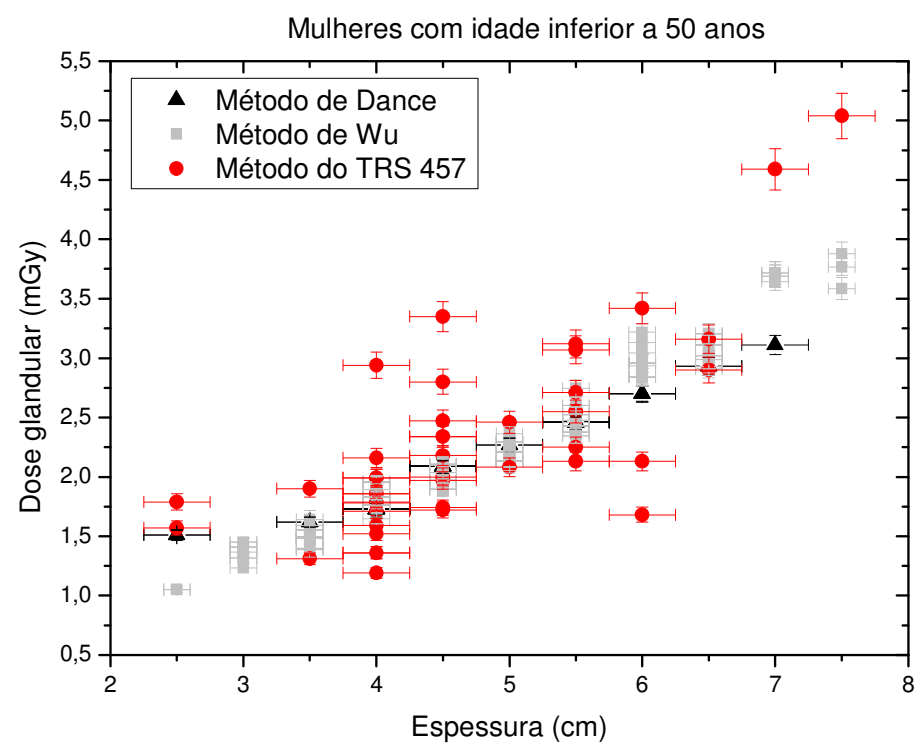

Figura 65 Comparação entre a estimativa de dose glandular média calculada pelos três métodos diferentes para cada espessura de mama comprimida estudada na projeção MLO no equipamento GE $700 \mathrm{~T}$, para mulheres com idade inferior a 50 anos. 


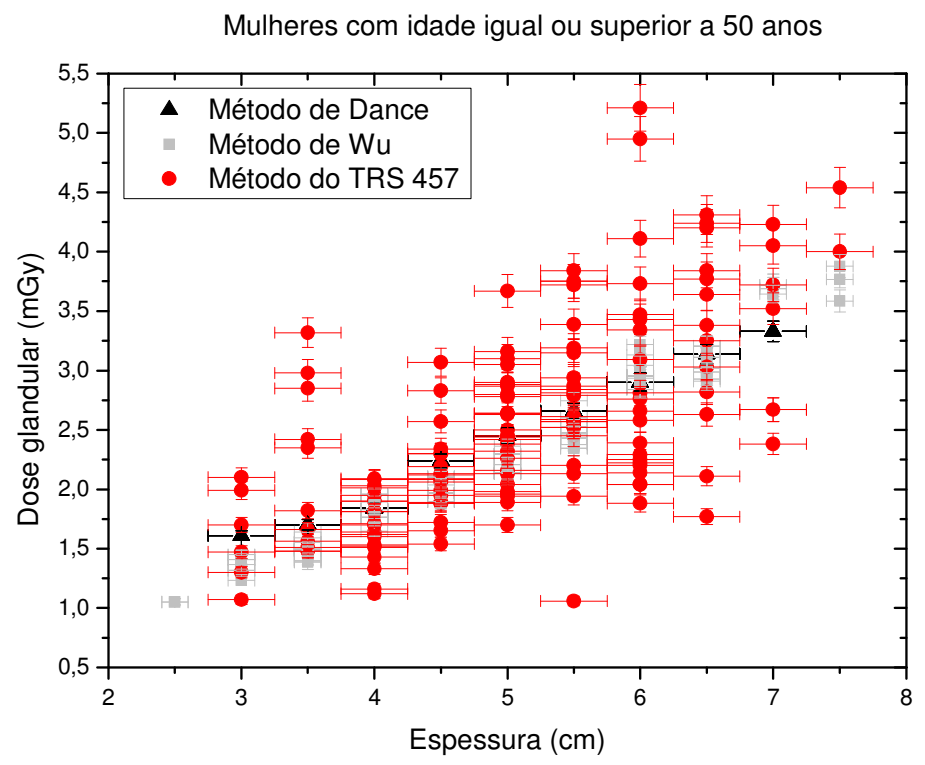

Figura 66 Comparação entre a estimativa de dose glandular média calculada pelos três métodos diferentes para cada espessura de mama comprimida estudada na projeção MLO no equipamento GE 700 T, para mulheres com idade igual ou superior a 50 anos.

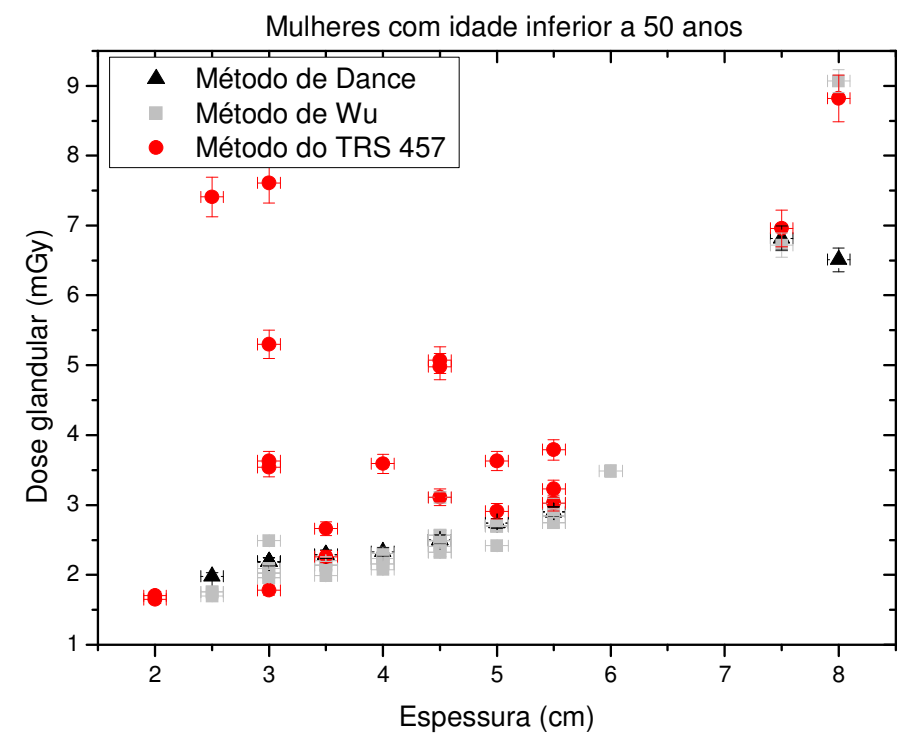

Figura 67 Comparação entre a estimativa de dose glandular média calculada pelos três métodos diferentes para cada espessura de mama comprimida estudada na projeção CC no equipamento Siemens - Mo/Mo, para mulheres com idade inferior a 50 anos. 


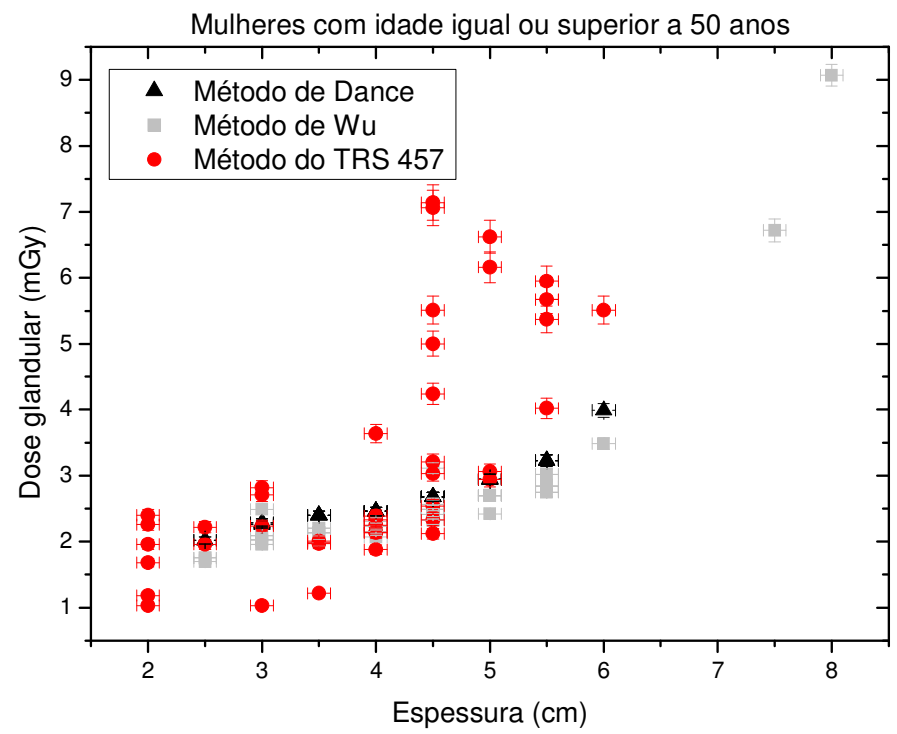

Figura 68 Comparação entre a estimativa de dose glandular média calculada pelos três métodos diferentes para cada espessura de mama comprimida estudada na projeção CC no equipamento Siemens - Mo/Mo, para mulheres com idade igual ou superior a 50 anos.

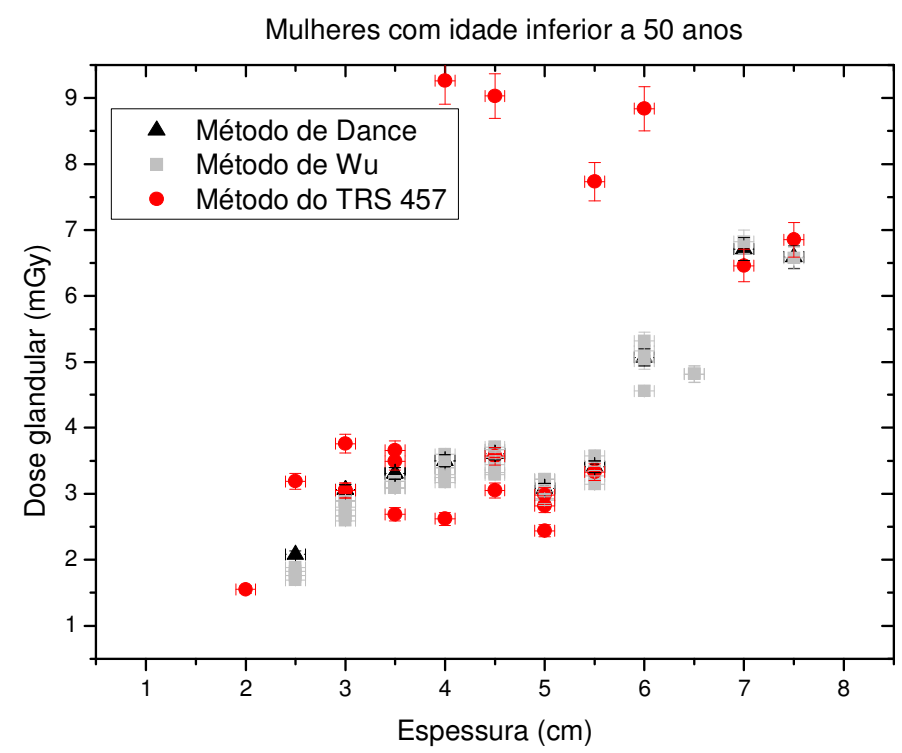

Figura 69 Comparação entre a estimativa de dose glandular média calculada pelos três métodos diferentes para cada espessura de mama comprimida estudada na projeção MLO no equipamento Siemens - Mo/Mo, para mulheres com idade inferior a 50 anos. 


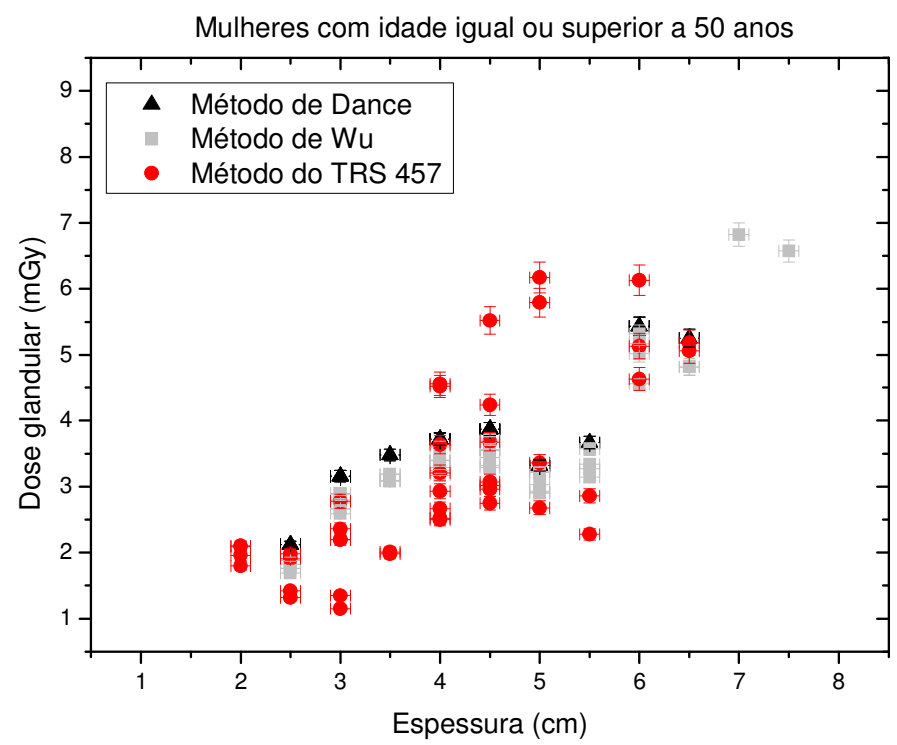

Figura 70 Comparação entre a estimativa de dose glandular média calculada pelos três métodos diferentes para cada espessura de mama comprimida estudada na projeção MLO no equipamento Siemens - Mo/Mo, para mulheres com idade igual ou superior a 50 anos.

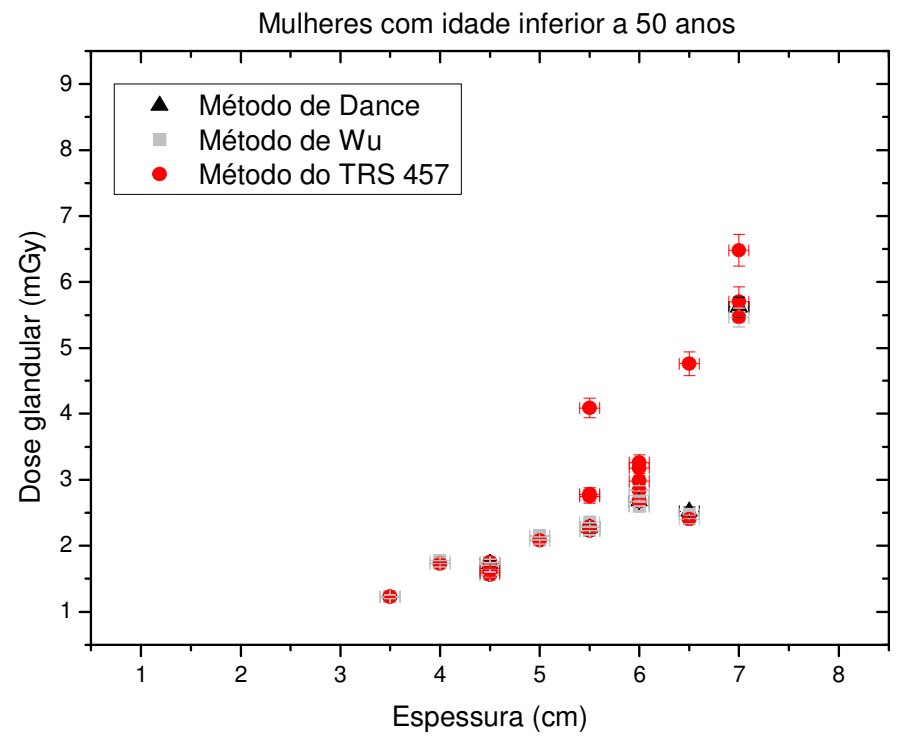

Figura 71 Comparação entre a estimativa de dose glandular média calculada pelos três métodos diferentes para cada espessura de mama comprimida estudada na projeção CC no equipamento Siemens - Mo/Rh, para mulheres com idade inferior a 50 anos. 


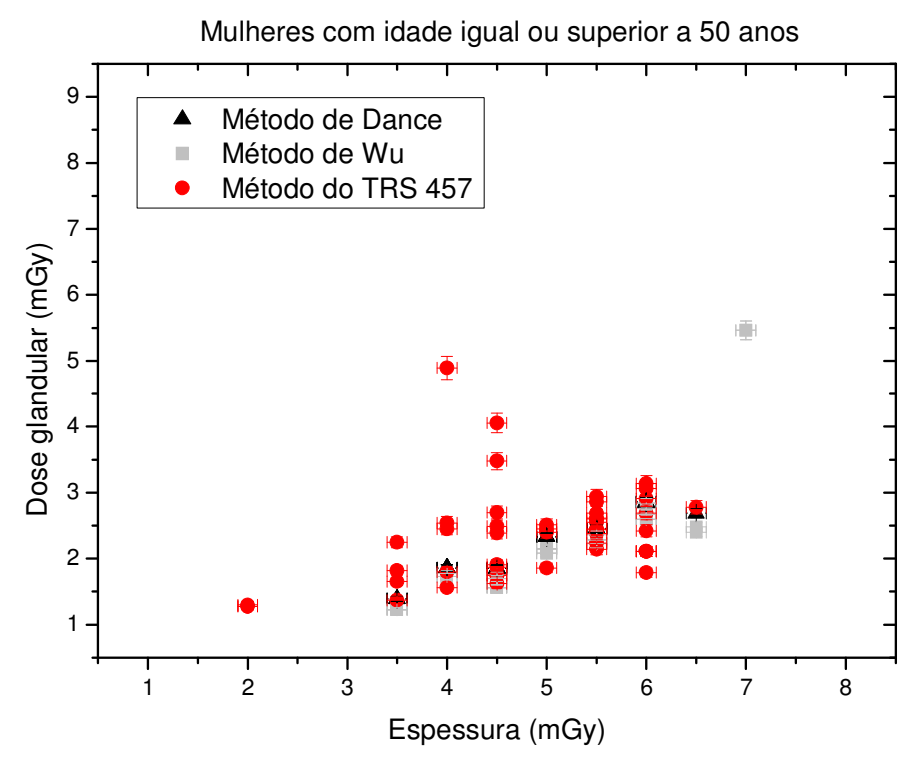

Figura 72 Comparação entre a estimativa de dose glandular média calculada pelos três métodos diferentes para cada espessura de mama comprimida estudada na projeção $\mathrm{CC}$ no equipamento Siemens - Mo/Rh, para mulheres com idade igual ou superior a 50 anos.

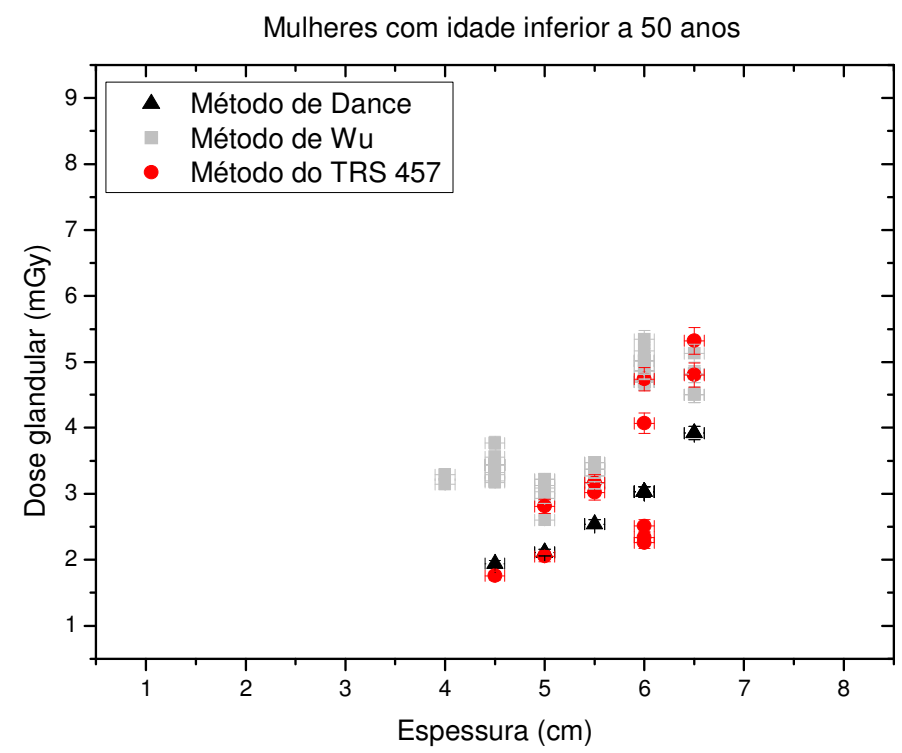

Figura 73 Comparação entre a estimativa de dose glandular média calculada pelos três métodos diferentes para cada espessura de mama comprimida estudada na projeção MLO no equipamento Siemens - Mo/Rh, para mulheres com idade inferior a 50 anos. 


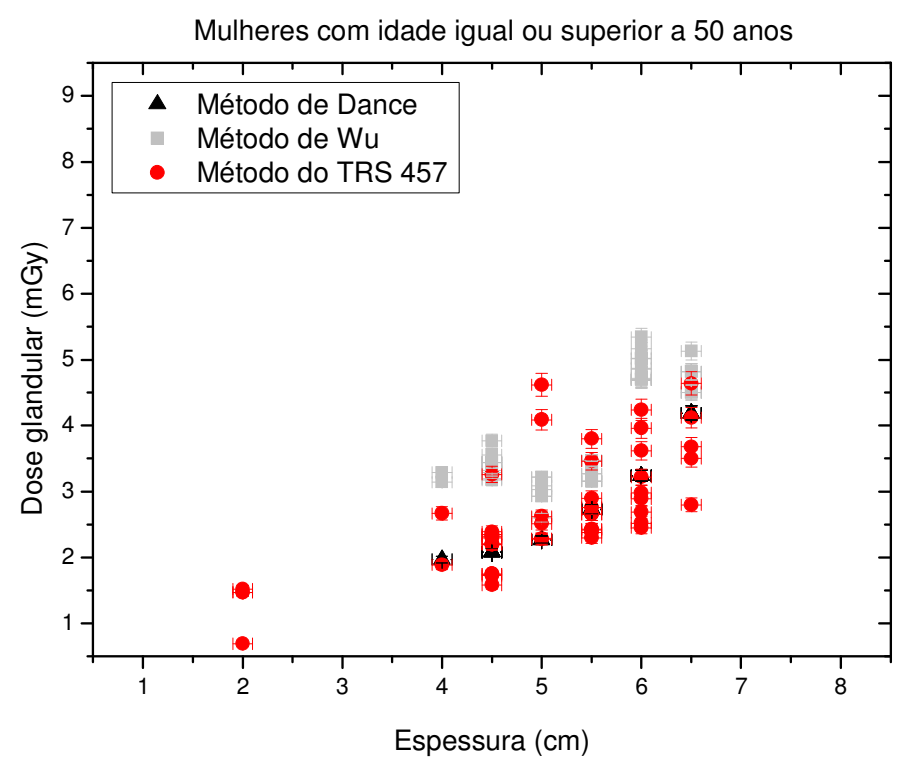

Figura 74 Comparação entre a estimativa de dose glandular média calculada pelos três métodos diferentes para cada espessura de mama comprimida estudada na projeção MLO no equipamento Siemens - Mo/Rh, para mulheres com idade igual ou superior a 50 anos.

No equipamento Siemens, para a combinação anodo-filtro Mo/Mo (Figuras 67 a 70), os valores de dose estimados pelos métodos de Dance ${ }^{17,18} \mathrm{e} \mathrm{Wu}^{19,20,21}$ são bem próximos, enquanto que os valores estimados pelo TRS $457^{22}$ giram em torno dos outros métodos. Para a combinação $\mathrm{Mo} / \mathrm{Rh}$, os valores de dose estimados pelos métodos de Dance ${ }^{17,18}$ e Wu ${ }^{19,20,21}$, na projeção CC (Figuras $71 \mathrm{e}$ 72) e , são praticamente coincidentes, enquanto que na projeção MLO (Figuras 73 e 74) os valores estimados pelo método de $\mathrm{Wu}^{19,20,21}$ são maiores que os estimados pelo método de Dance ${ }^{17,18}$. Os valores de dose estimados pelo método do TRS $457^{22}$ giram em torno dos valores apresentados pelos outros métodos.

No equipamento GE DS, em ambas as combinações anodo-filtro (Figuras 75 a 82), observa-se que os valores estimados pelo método de Dance ${ }^{17,18}$ são um pouco maiores que os estimados pelo método de $\mathrm{Wu}^{19,20,21}$, essa diferença não passa de 0,3 mGy. Enquanto que os valores estimados pelo método do TRS $457^{22}$ giram em torno dos valores estimados pelos outros métodos, mas nota-se que estes valores são, na maioria, maiores. 


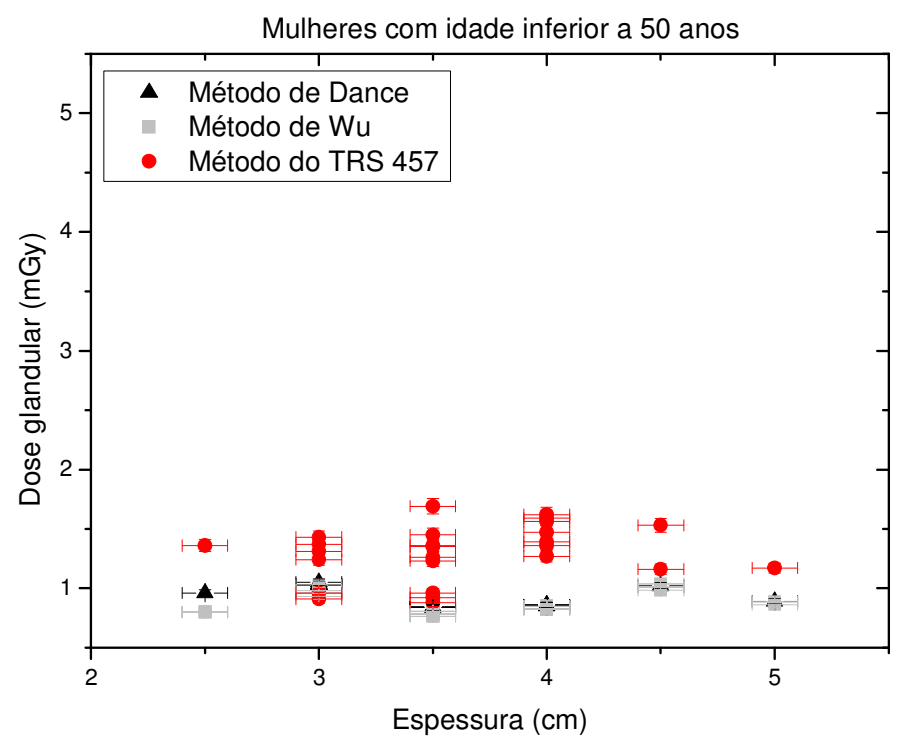

Figura 75 Comparação entre a estimativa de dose glandular média calculada pelos três métodos diferentes para cada espessura de mama comprimida estudada na projeção CC no equipamento GE DS - Mo/Rh, para mulheres com idade inferior a 50 anos.

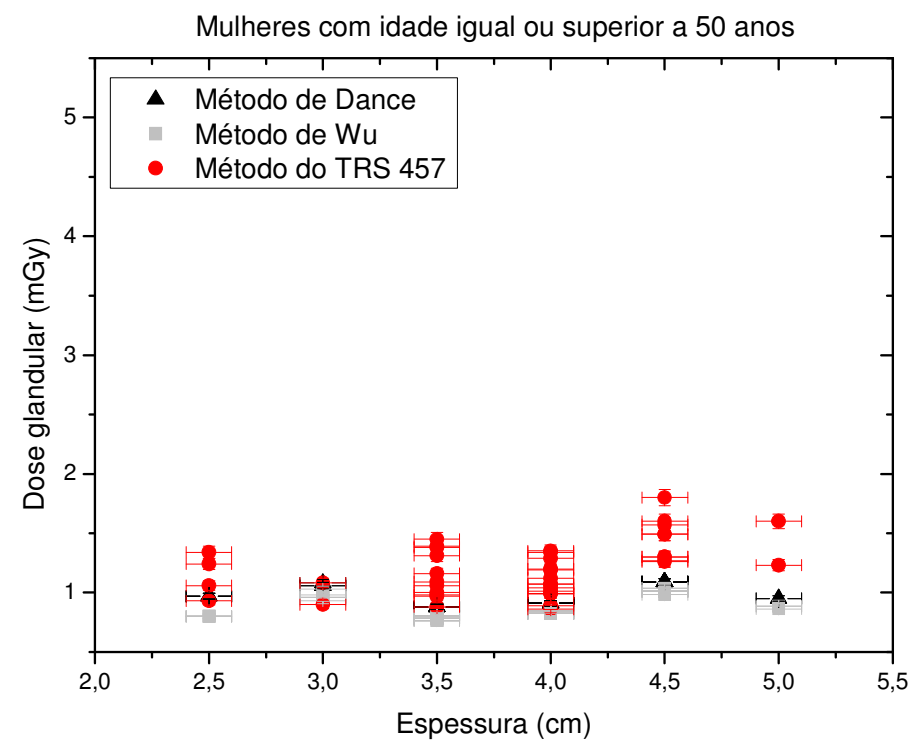

Figura 76 Comparação entre a estimativa de dose glandular média calculada pelos três métodos diferentes para cada espessura de mama comprimida estudada na projeção CC no equipamento GE DS - Mo/Rh, para mulheres com idade igual ou superior a 50 anos. 


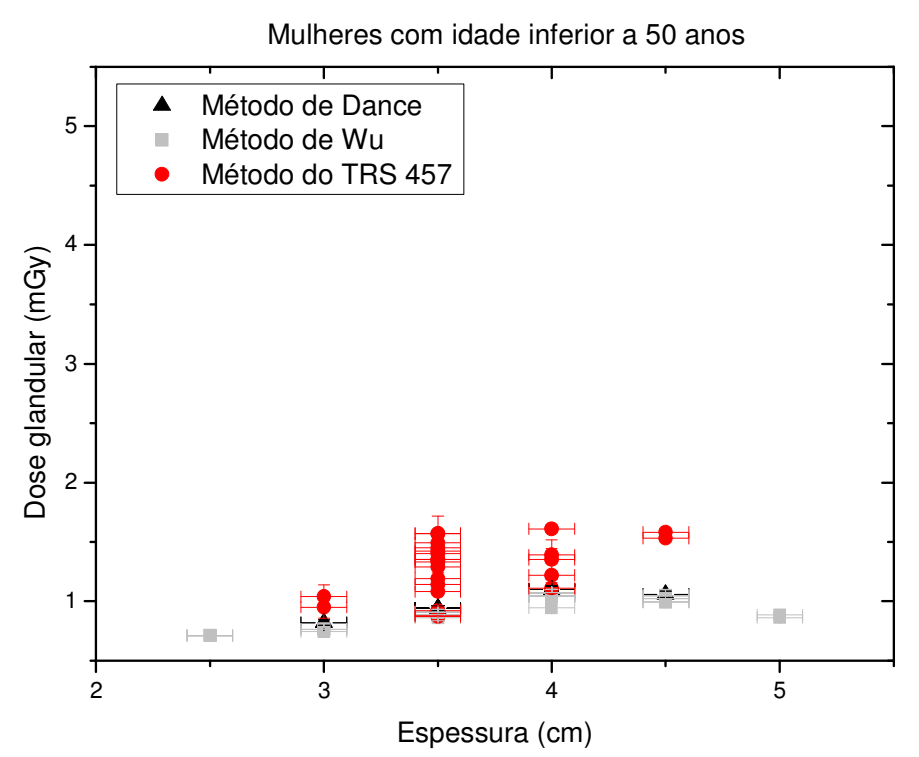

Figura 77 Comparação entre a estimativa de dose glandular média calculada pelos três métodos diferentes para cada espessura de mama comprimida estudada na projeção MLO no equipamento GE DS - Mo/Rh, para mulheres com idade inferior a 50 anos.

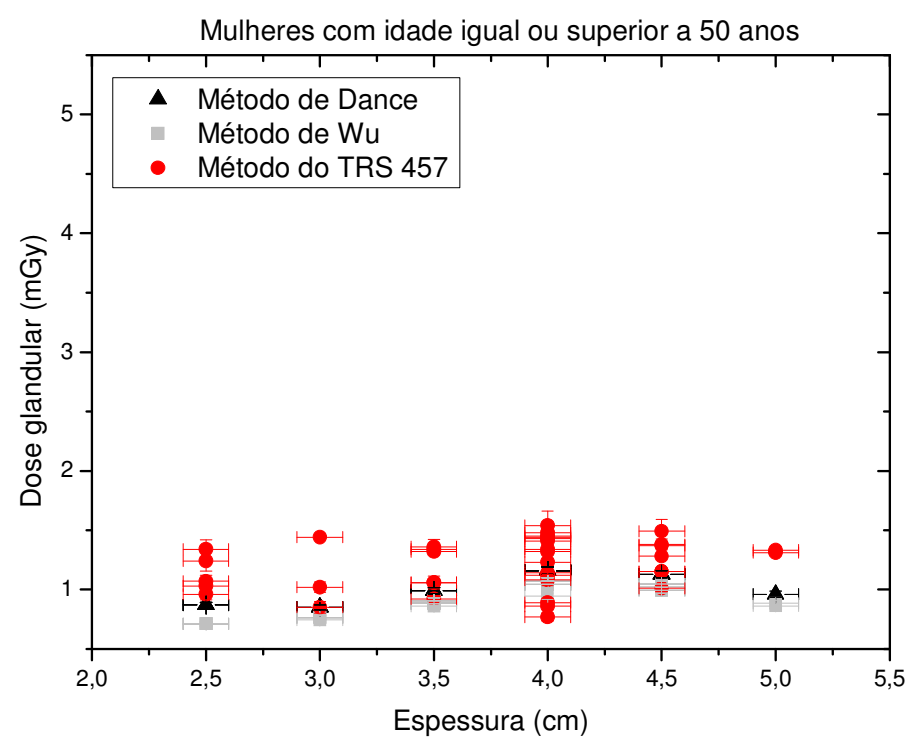

Figura 78 Comparação entre a estimativa de dose glandular média calculada pelos três métodos diferentes para cada espessura de mama comprimida estudada na projeção MLO no equipamento GE DS - Mo/Rh, para mulheres com idade igual ou superior a 50 anos. 


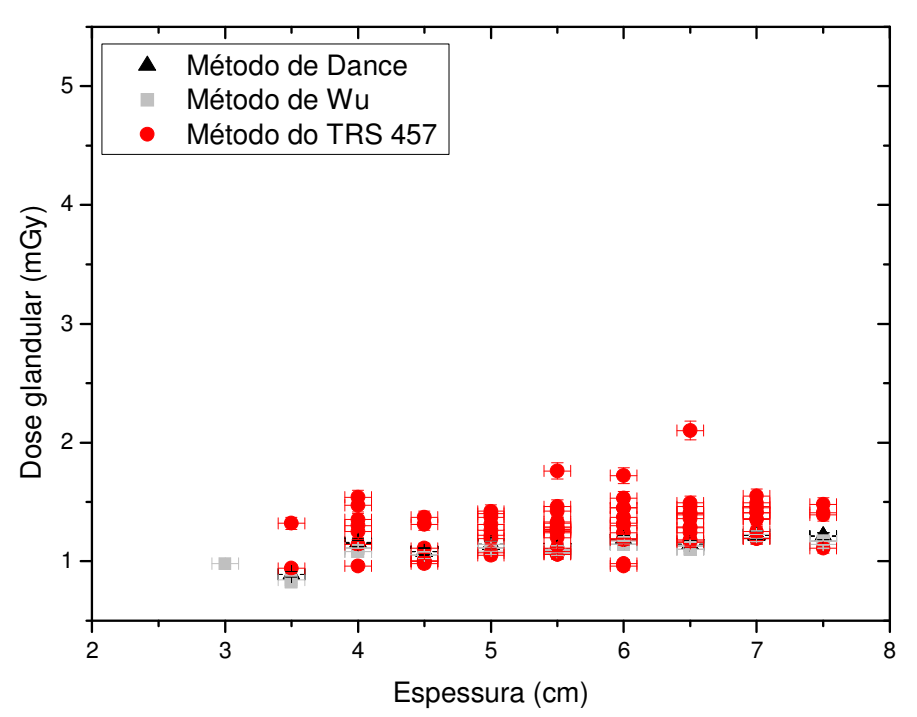

Figura 79 Comparação entre a estimativa de dose glandular média calculada pelos três métodos diferentes para cada espessura de mama comprimida estudada na projeção CC no equipamento GE DS - Rh/Rh, para mulheres com idade inferior a 50 anos.

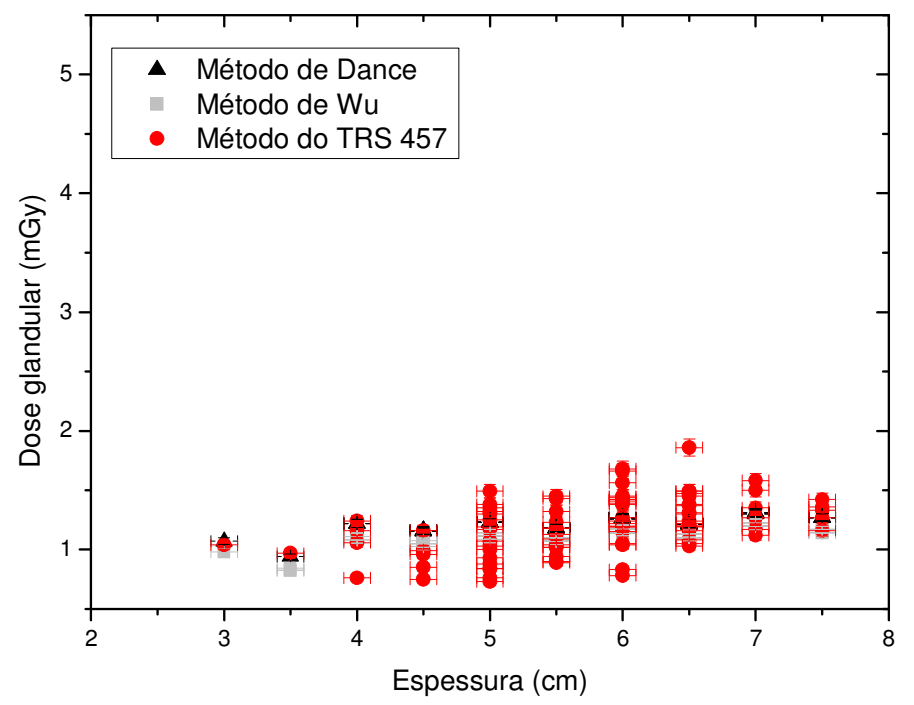

Figura 80 Comparação entre a estimativa de dose glandular média calculada pelos três métodos diferentes para cada espessura de mama comprimida estudada na projeção CC no equipamento GE DS - Rh/Rh, para mulheres com idade igual ou superior a 50 anos. 


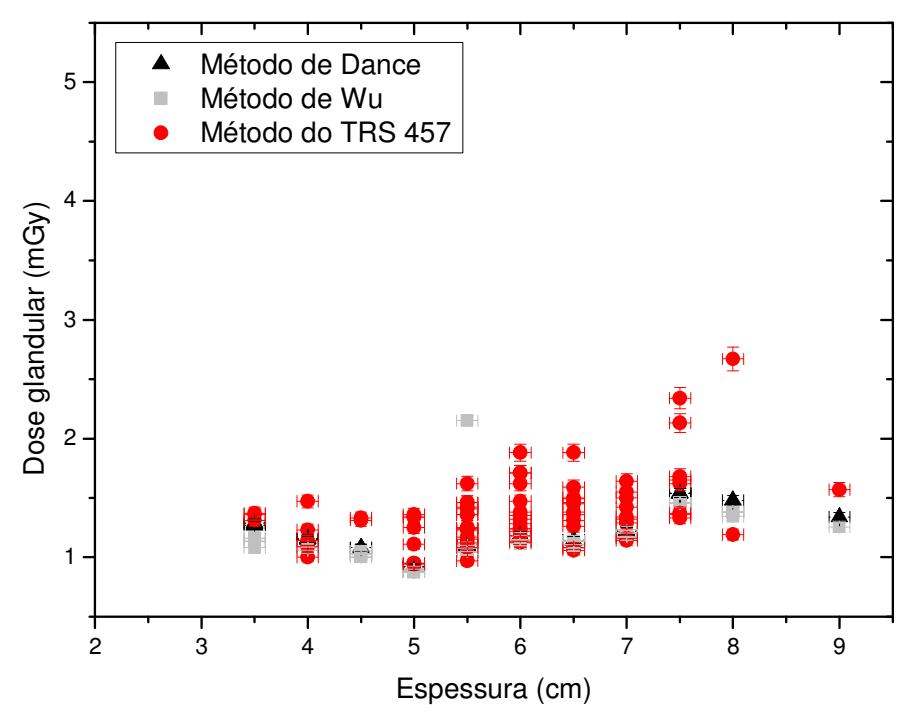

Figura 81 Comparação entre a estimativa de dose glandular média calculada pelos três métodos diferentes para cada espessura de mama comprimida estudada na projeção MLO no equipamento GE DS - Rh/Rh, para mulheres com idade inferior a 50 anos.

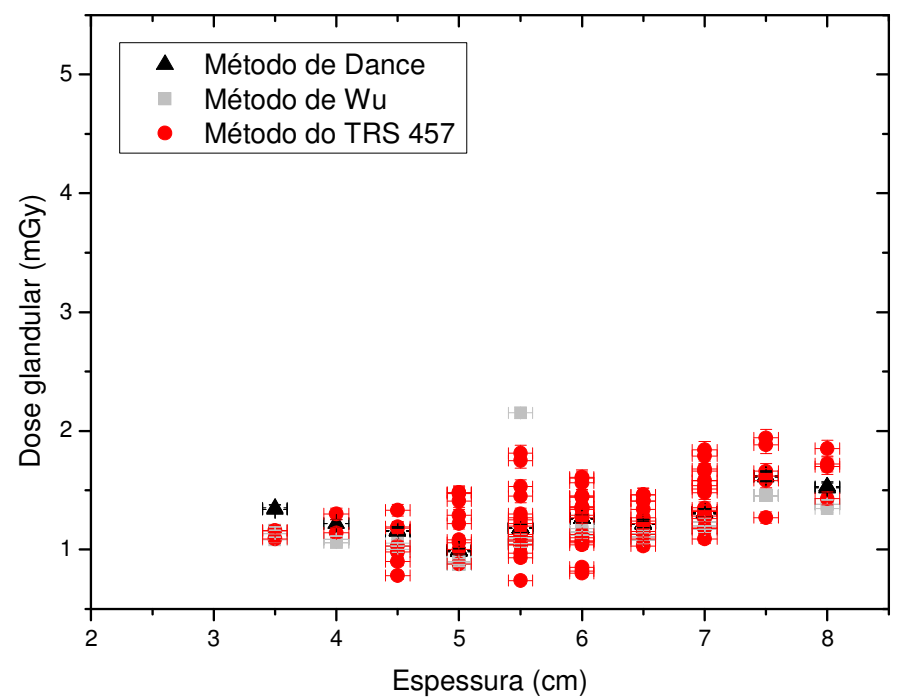

Figura 82 Comparação entre a estimativa de dose glandular média calculada pelos três métodos diferentes para cada espessura de mama comprimida estudada na projeção MLO no equipamento GE DS - Rh/Rh, para mulheres com idade igual ou superior a 50 anos. 


\subsubsection{Comparação entre os três mamógrafos}

Ao comparar os resultados da dose glandular estimados tanto pelo método de Dance $^{17,18}$ (Figuras 83 a 86) quanto pelo de $\mathrm{Wu}^{19,20,21}$ (Figuras 87 e 88 ) entre os equipamentos Siemens e GE DS, na combinação anodo-filtro Mo/Rh, observa-se que os valores obtidos no equipamento Siemens são sempre maiores que os valores de dose do GE DS. Nota-se também que os valores de dose obtidos no Siemens aumentam à medida que a espessura de mama comprimida aumenta, o que não ocorre para o equipamento GE DS, onde os valores se mantém praticamente constantes.

Observa-se praticamente o mesmo comportamento ao realizar esta mesma comparação entre os resultados das doses glandulares estimados pelo método do TRS $457^{22}$ (Figuras 89 a 92), porém existem alguns poucos valores coincidentes.

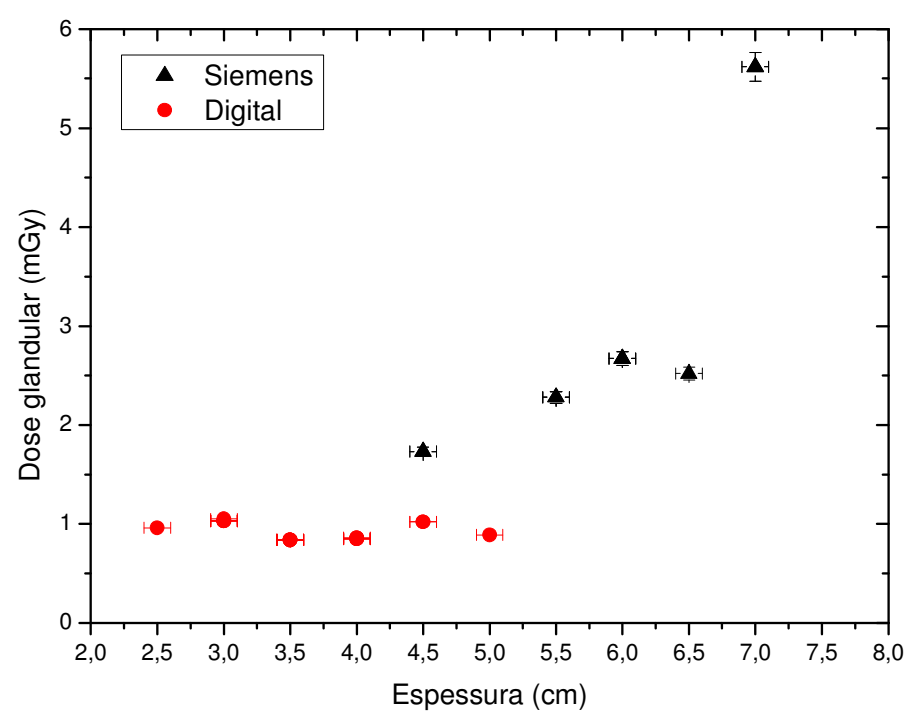

Figura 83 Comparação entre as estimativas de DGM calculada pelo método de Dance para cada espessura de mama comprimida estudada na projeção CC nos equipamentos Siemens e GE DS - MoRh para mulheres com idade inferior a 50 anos. 


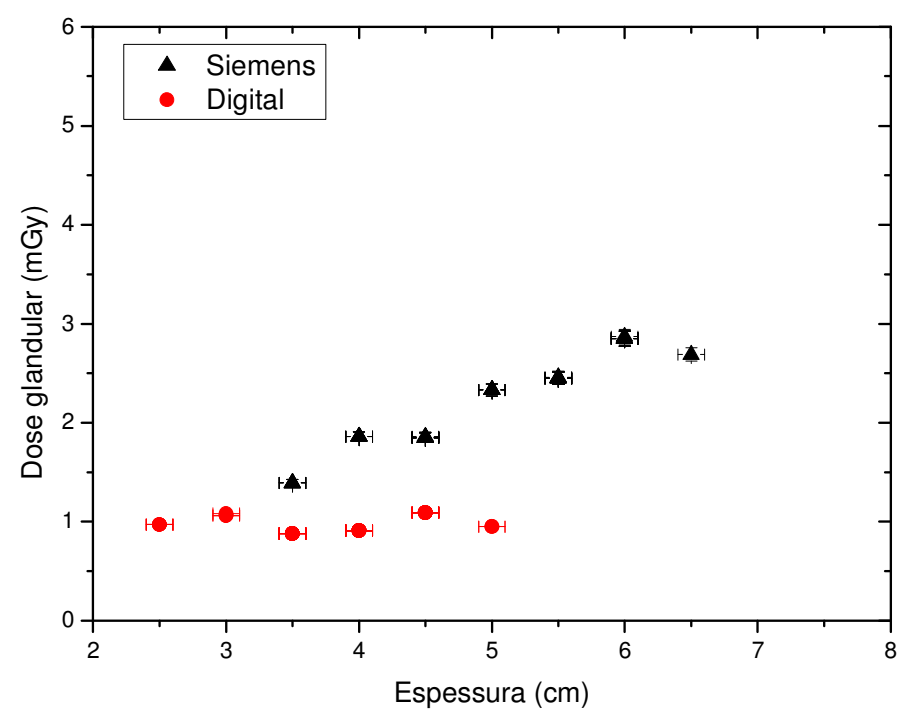

Figura 84 Comparação entre as estimativas de DGM calculada pelo método de Dance para cada espessura de mama comprimida estudada na projeção CC nos equipamentos Siemens e GE DS - MoRh para mulheres com idade igual ou superior a 50 anos.

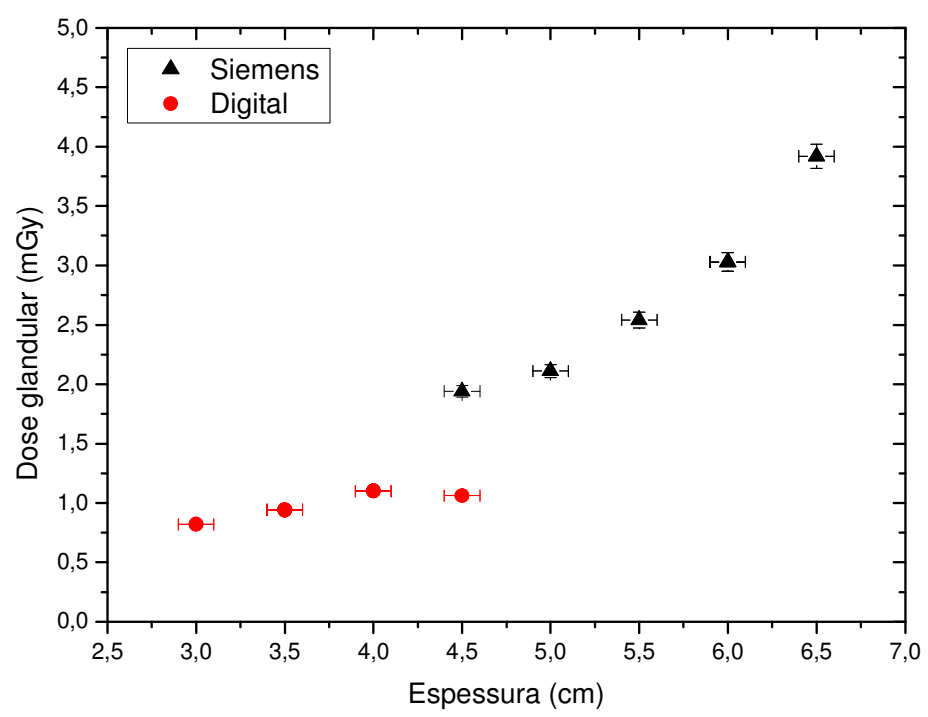

Figura 85 Comparação entre as estimativas de DGM calculada pelo método de Dance para cada espessura de mama comprimida estudada na projeção MLO nos equipamentos Siemens e GE DS - MoRh para mulheres com idade inferior a 50 anos. 


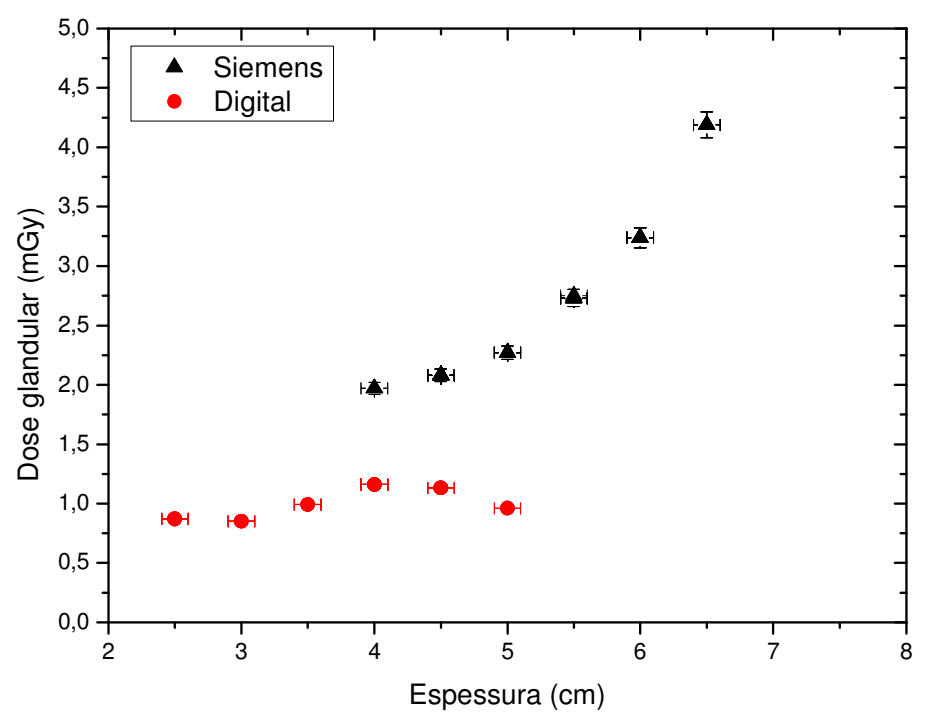

Figura 86 Comparação entre as estimativas de DGM calculada pelo método de Dance para cada espessura de mama comprimida estudada na projeção MLO nos equipamentos Siemens e GE DS - MoRh para mulheres com idade igual ou superior a 50 anos.

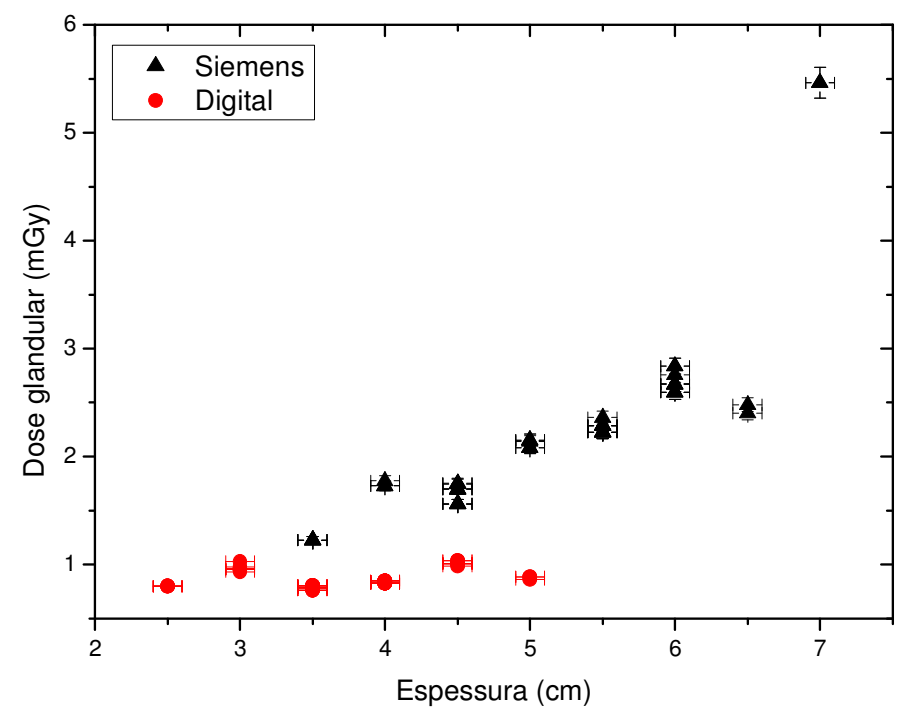

Figura 87 Comparação entre as estimativas de DGM calculada pelo método de Wu para cada espessura de mama comprimida estudada na projeção CC nos equipamentos Siemens e GE DS - MoRh. 


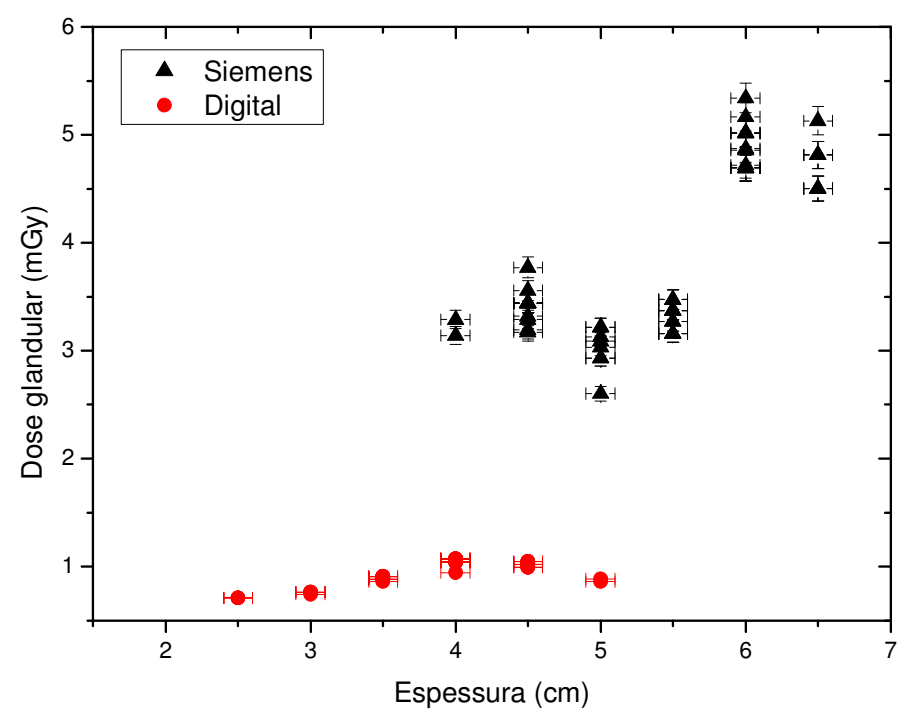

Figura 88 Comparação entre as estimativas de DGM calculada pelo método de Wu para cada espessura de mama comprimida estudada na projeção MLO nos equipamentos Siemens e GE DS - MoRh.

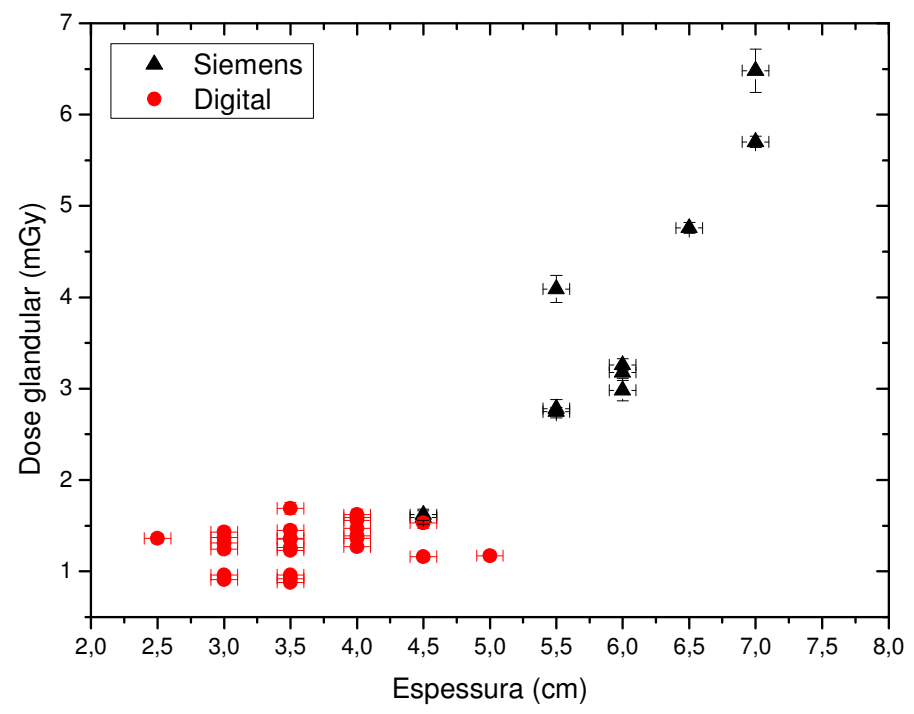

Figura 89 Comparação entre as estimativas de DGM calculada pelo método do TRS 457 para cada espessura de mama comprimida estudada na projeção CC nos equipamentos Siemens e GE DS - MoRh para mulheres com idade inferior a 50 anos. 


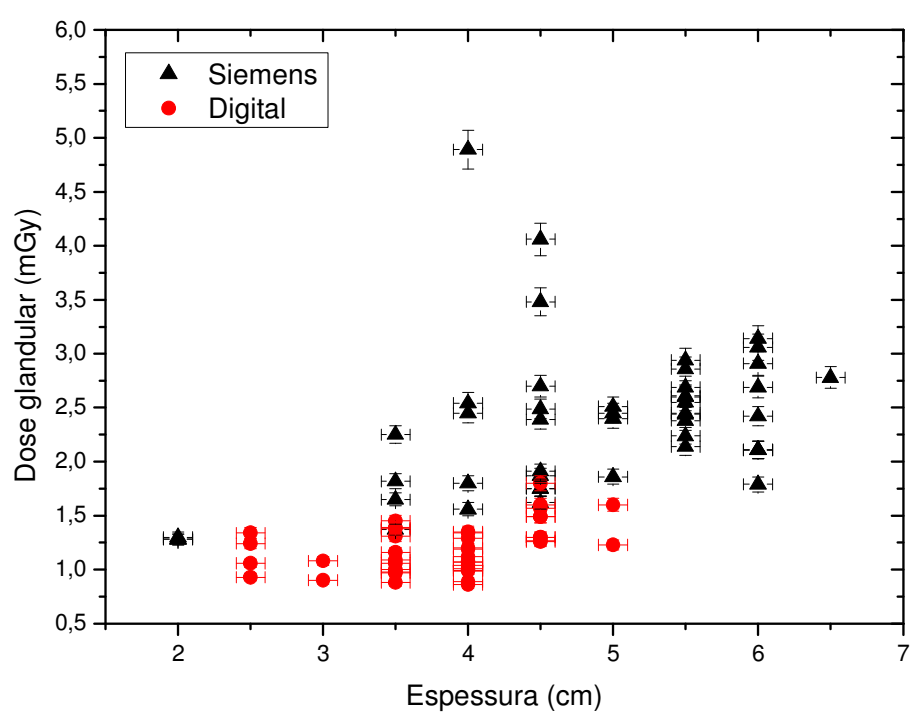

Figura 90 Comparação entre as estimativas de DGM calculada pelo método do TRS 457 para cada espessura de mama comprimida estudada na projeção CC nos equipamentos Siemens e GE DS - MoRh para mulheres com idade igual ou superior a 50 anos.

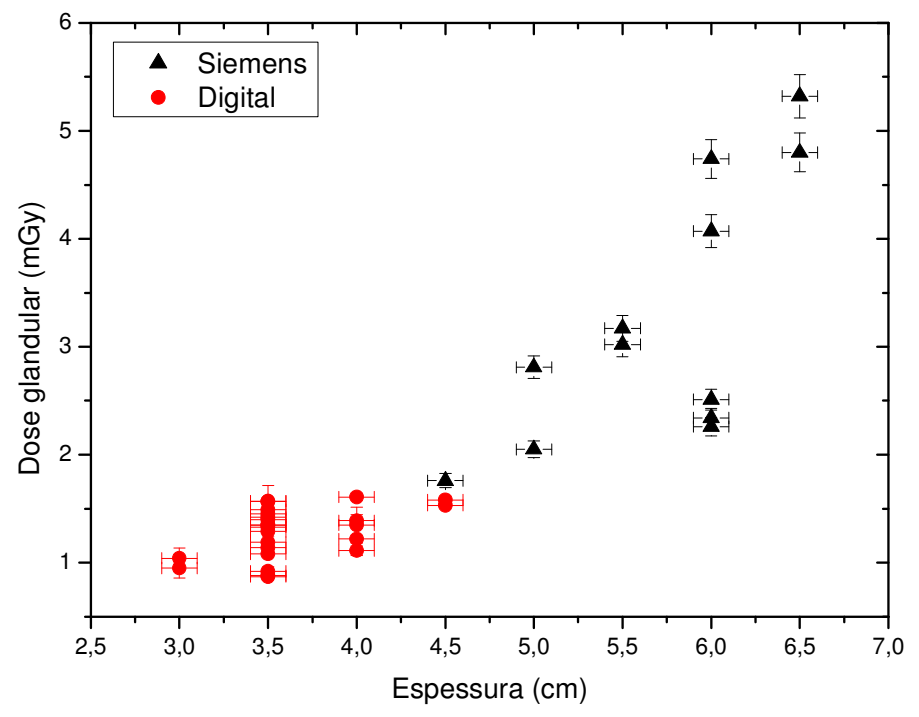

Figura 91 Comparação entre as estimativas de DGM calculada pelo método do TRS 457 para cada espessura de mama comprimida estudada na projeção MLO nos equipamentos Siemens e GE DS - MoRh para mulheres com idade inferior a 50 anos. 




Figura 92 Comparação entre as estimativas de DGM calculada pelo método do TRS 457 para cada espessura de mama comprimida estudada na projeção MLO nos equipamentos Siemens e GE DS - MoRh para mulheres com idade igual ou superior a 50 anos.

Para a combinação anodo-filtro Mo/Mo (Figuras 93 a 102), os valores de dose glandular média estimados por qualquer método, no equipamento Siemens, são sempre maiores que os valores obtidos no equipamento GE $700 \mathrm{~T}$, em todas as espessuras, para qualquer idade das pacientes. Alguns valores estimados pelo método do TRS $457^{22}$ obtidos no equipamento Siemens se igualam ou são menores que os valores obtidos no equipamento GE $700 \mathrm{~T}$, mas a maioria dos valores são maiores quando obtidos no Siemens.

Na projeção CC, os valores de dose estimados pelos métodos de Dance ${ }^{17,18}$

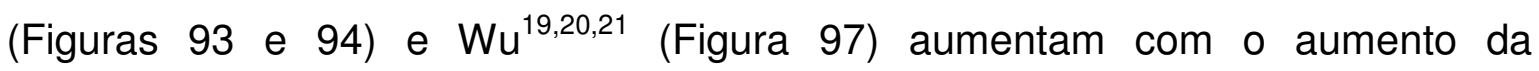
espessura. O mesmo resultado é observado na projeção MLO (Figuras 95, 96 e 98), com exceção das espessuras $5,5,5$ e $6 \mathrm{~cm}$. Tendo em vista que os valores de dose estimados pelo método do TRS $457^{22}$ referem-se a cada paciente que realizou o exame, os gráficos apresentados na Figuras 99 a 102 apresentam muitos dados. Isso dificulta a análise do resultado, porém é fácil notar que a maioria dos valores obtidos no equipamento Siemens são maiores que os valores obtidos no GE $700 \mathrm{~T}$. 


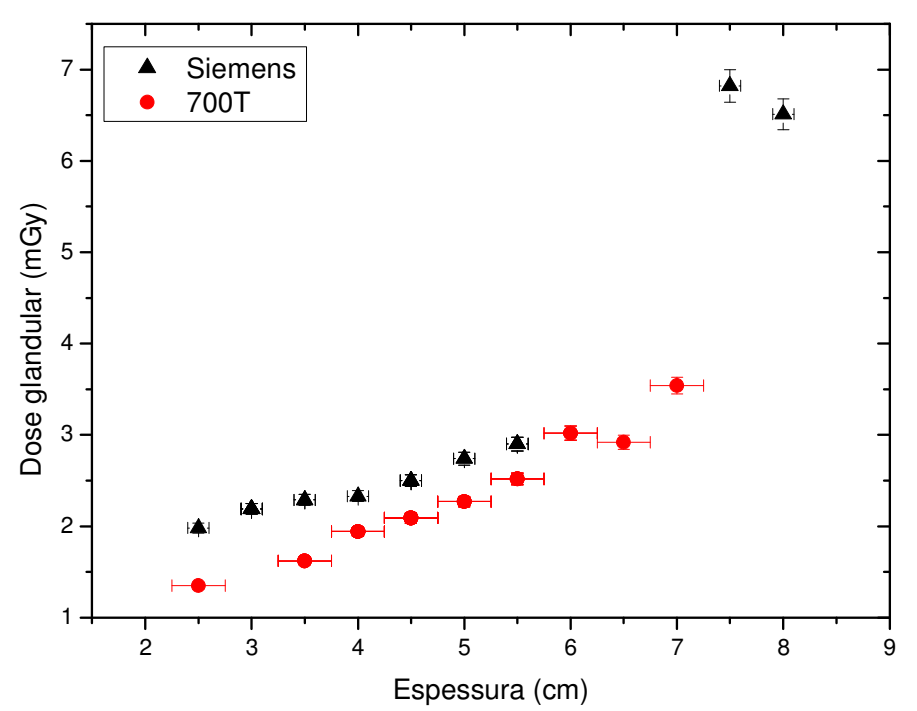

Figura 93 Comparação entre as estimativas de DGM calculada pelo método de Dance para cada espessura de mama comprimida estudada na projeção CC nos equipamentos Siemens e GE 700 T - MoMo para mulheres com idade inferior a 50 anos.

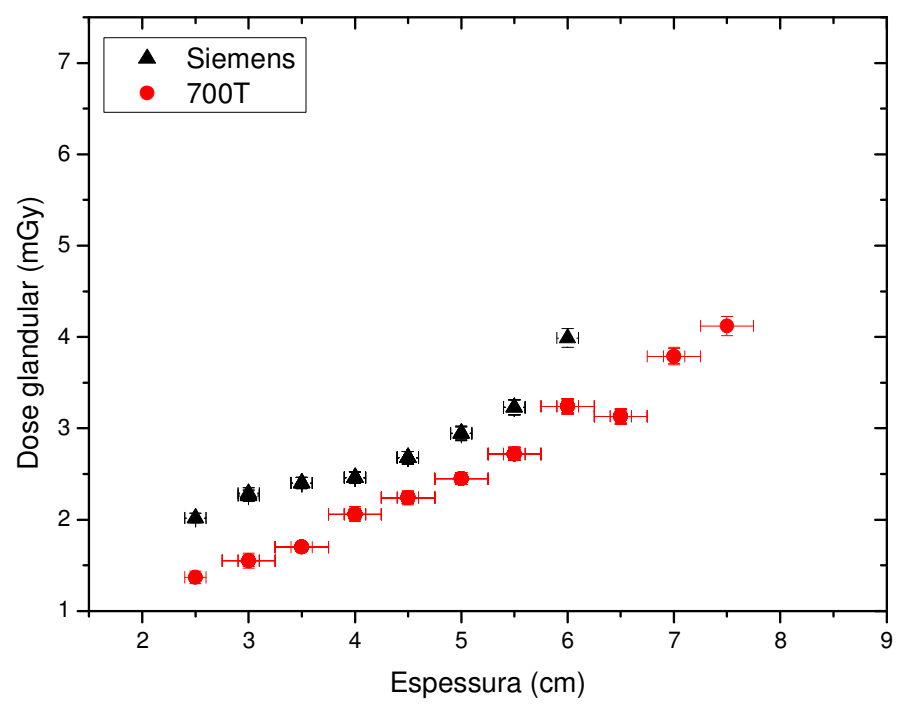

Figura 94 Comparação entre as estimativas de DGM calculada pelo método de Dance para cada espessura de mama comprimida estudada na projeção CC nos equipamentos Siemens e GE 700 T - MoMo para mulheres com idade igual ou superior a 50 anos. 


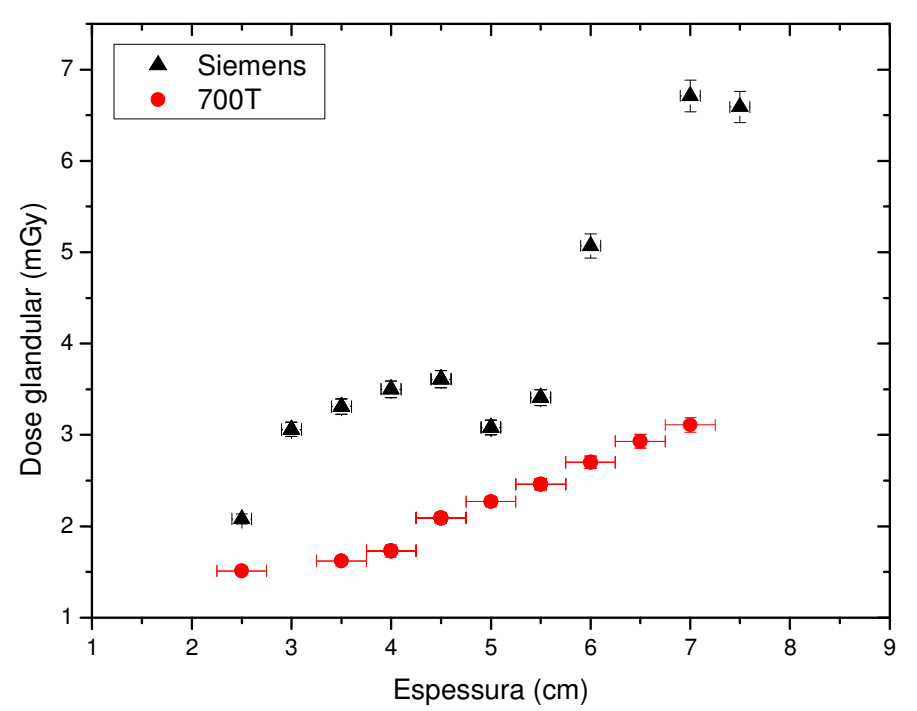

Figura 95 Comparação entre as estimativas de DGM calculada pelo método de Dance para cada espessura de mama comprimida estudada na projeção MLO nos equipamentos Siemens e GE 700 T - MoMo para mulheres com idade inferior a 50 anos.

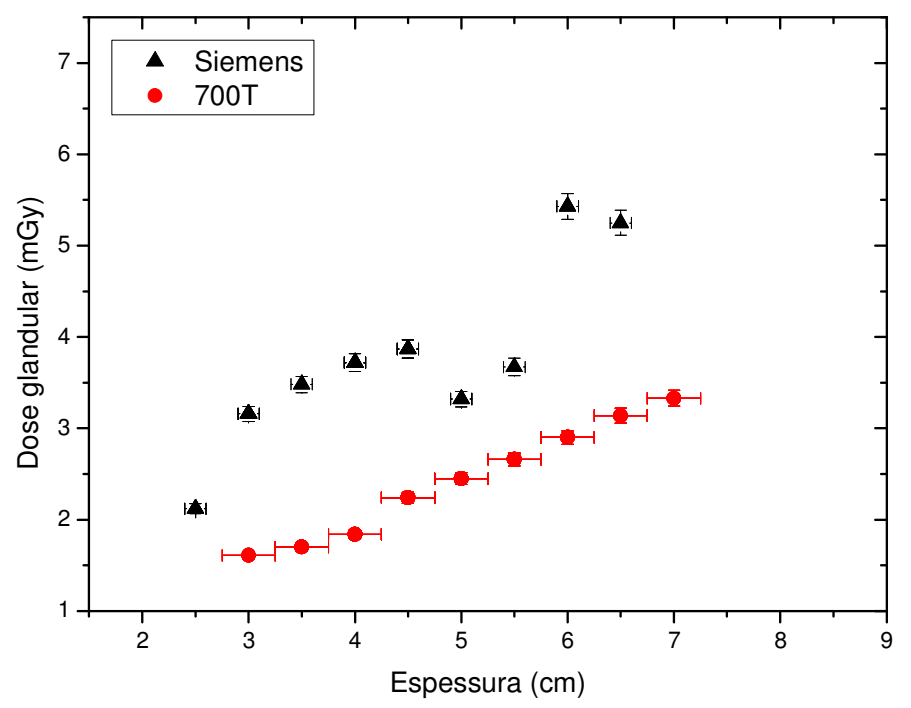

Figura 96 Comparação entre as estimativas de DGM calculada pelo método de Dance para cada espessura de mama comprimida estudada na projeção MLO nos equipamentos Siemens e GE 700 T - MoMo para mulheres com idade igual ou superior a 50 anos. 


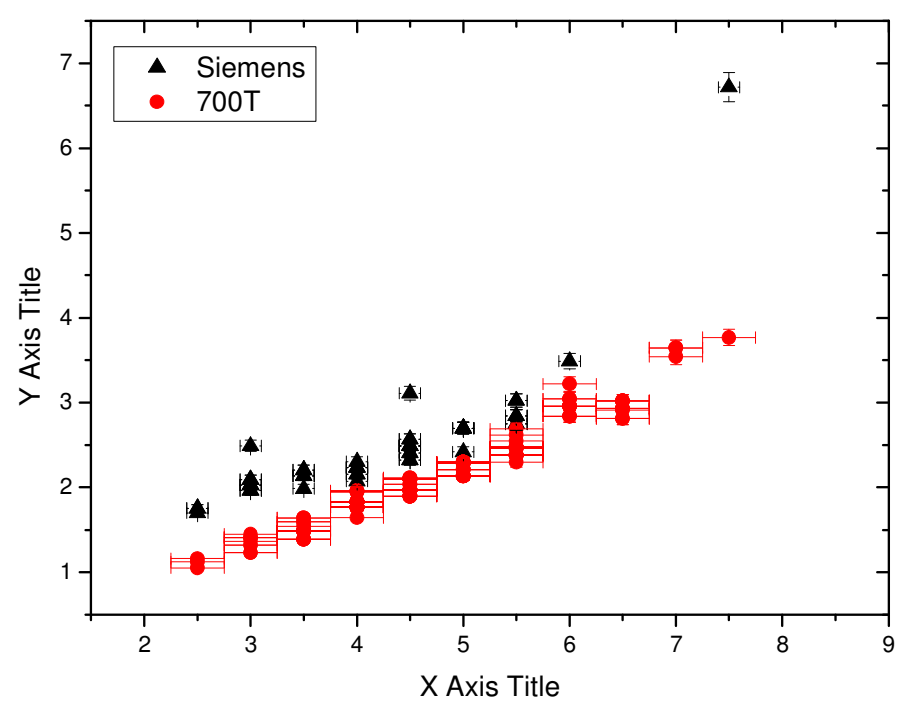

Figura 97 Comparação entre as estimativas de DGM calculada pelo método de Wu para cada espessura de mama comprimida estudada na projeção CC nos equipamentos Siemens e GE 700 T - MoMo.

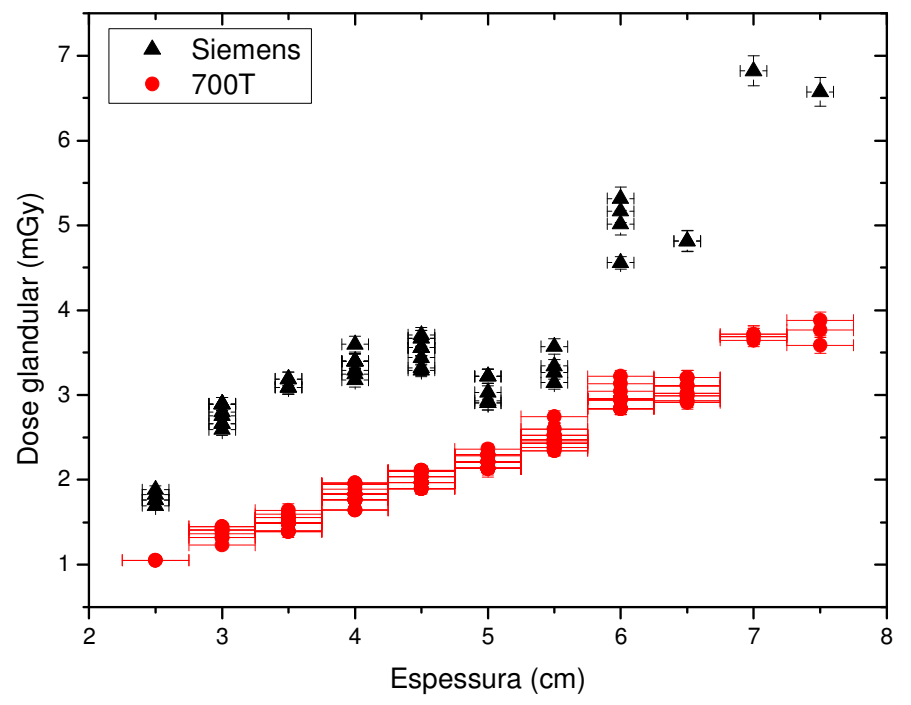

Figura 98 Comparação entre as estimativas de DGM calculada pelo método de Wu para cada espessura de mama comprimida estudada na projeção MLO nos equipamentos Siemens e GE 700 T - MoMo. 


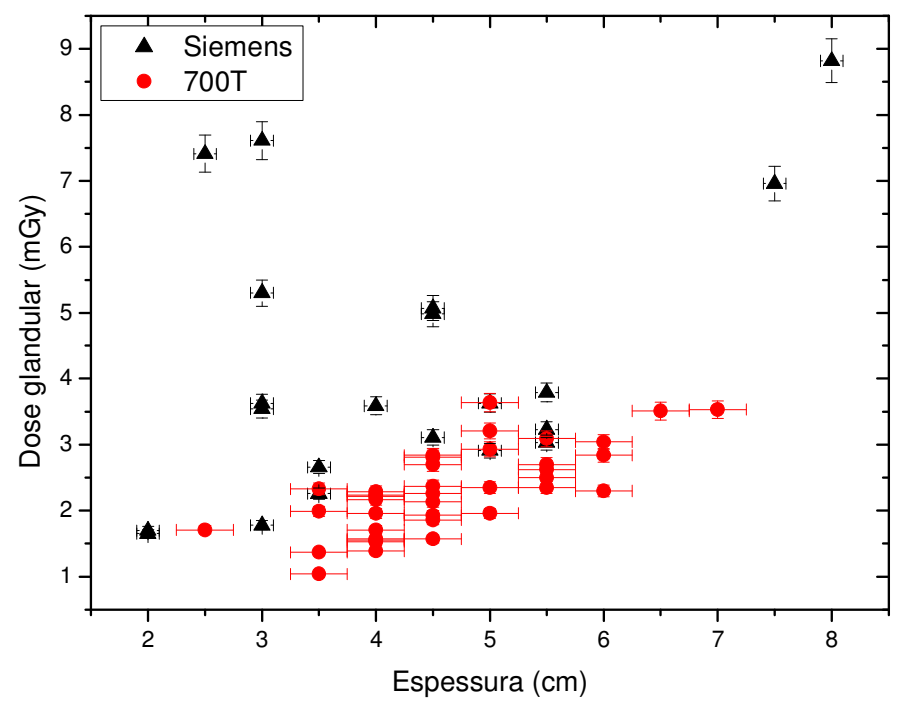

Figura 99 Comparação entre as estimativas de DGM calculada pelo método do TRS 457 para cada espessura de mama comprimida estudada na projeção CC nos equipamentos Siemens e GE 700 T - MoMo para mulheres com idade inferior a 50 anos.

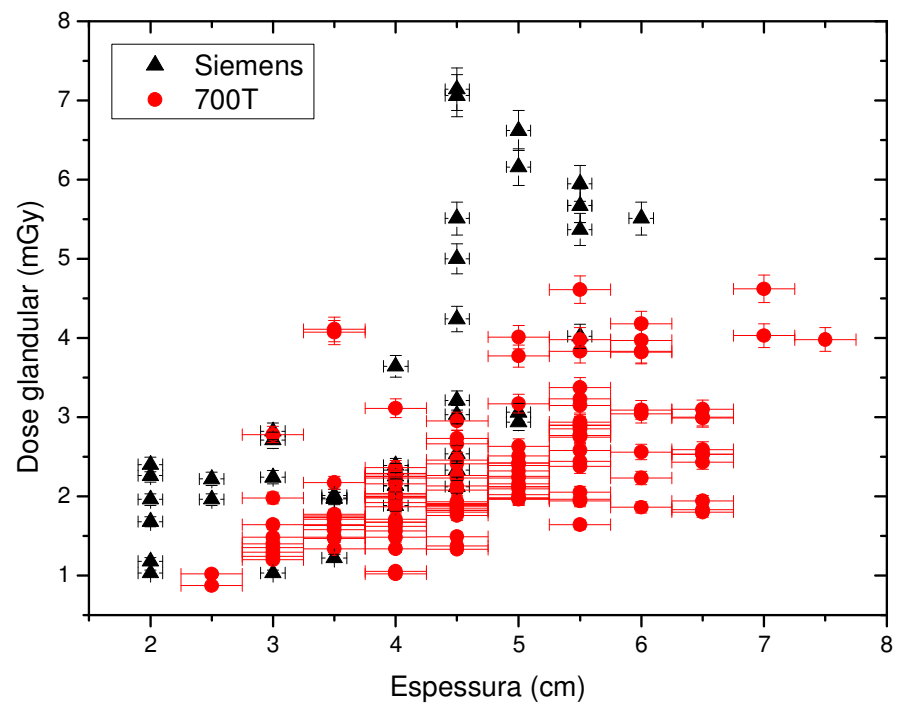

Figura 100 Comparação entre as estimativas de DGM calculada pelo método do TRS 457 para cada espessura de mama comprimida estudada na projeção CC nos equipamentos Siemens e GE 700 T - MoMo para mulheres com idade igual ou superior a 50 anos. 


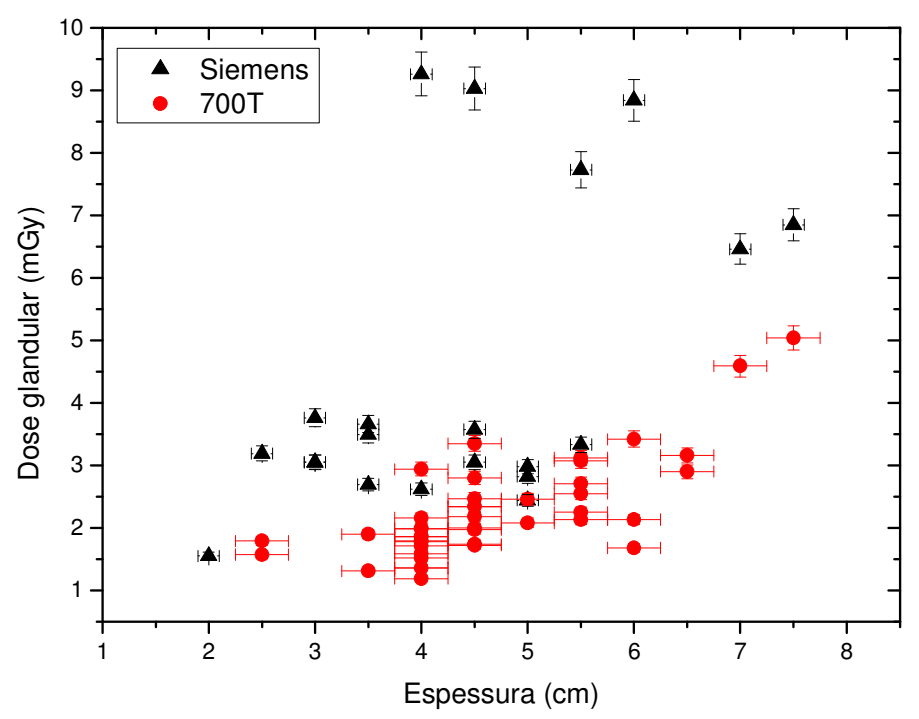

Figura 101 Comparação entre as estimativas de DGM calculada pelo método do TRS 457 para cada espessura de mama comprimida estudada na projeção MLO nos equipamentos Siemens e GE 700 T - MoMo para mulheres com idade inferior a 50 anos.

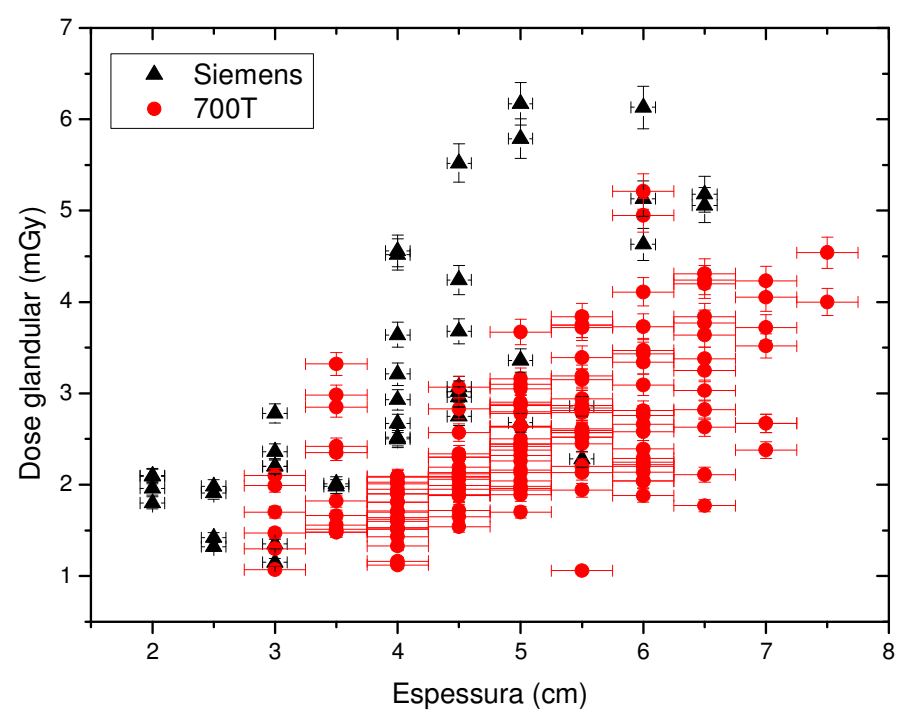

Figura 102 Comparação entre as estimativas de DGM calculada pelo método do TRS 457 para cada espessura de mama comprimida estudada na projeção MLO nos equipamentos Siemens e GE 700 T - MoMo para mulheres com idade igual ou superior a 50 anos. 


\subsection{Discussão dos resultados}

Ao realizar o posicionamento da paciente na projeção $\mathrm{CC}$, as técnicas procuravam não deixar que o músculo peitoral aparecesse na imagem, para atender à exigência de um dos radiologistas. O aparecimento do músculo peitoral na margem da imagem da projeção CC é um dos critérios de qualidade apresentados pelo guia europeu, pois garante que toda a mama esteja presente na imagem.

A processadora da mamografia é dedicada, o que facilita que se obtenha uma melhor qualidade da imagem, visto que os seus parâmetros são ajustados para atender exclusivamente aos filmes mamográficos.

O departamento faz o controle diário das condições da processadora antes de começar a atender às pacientes. Esse controle é bastante rigoroso e se a processadora apresentar alguma avaria, por menor que seja, o radiologista chefe não permite que sejam realizados exames enquanto o problema não seja solucionado. Essa atitude é excelente, visto que a processadora pode causar a perda da qualidade da imagem obtida nos mamógrafos convencionais.

Os equipamentos GE 700 T e GE DS não têm a mesa adequada para a aquisição de imagem de mamas grandes. Portanto as técnicas são obrigadas a realizar o exame no Siemens, apesar de muitas vezes o equipamento mais indicado ser o GE DS, visto que ele contém a combinação anodo-filtro $R h / R h$. Por isso, a média das doses glandulares para mulheres com mama grande é elevada. Para este problema não há uma solução simples, pois o equipamento GE $700 \mathrm{~T}$ contém apenas a combinação anodo-filtro Mo/Mo, que não é indicada para mamas espessas e, não há disponibilidade, no mercado, de mesa para este equipamento digital, que atenda às mamas grandes.

Por fatores burocráticos, pacientes que utilizam convênio para realizar a mamografia, não podem realizar a aquisição da imagem no equipamento GE DS, apenas as pacientes do Sistema Único de Saúde. A desvantagem deste acordo burocrático é que mesmo que a mama da paciente tenha características, cuja imagem apresentasse a melhor qualidade no equipamento GE DS, este não pode ser usado.

Foi observado, durante o acompanhamento da rotina do departamento, que boa parte das técnicas não tinham conhecimento sobre a diferença entre as 
combinações anodo-filtro. Portanto, escolhiam sempre a mesma combinação para qualquer tipo e tamanho de mama da paciente. A escolha inadequada da combinação anodo-filtro pode causar altas doses na paciente ${ }^{11}$.

Os equipamentos GE $700 \mathrm{~T}$ e Siemens são semi-automáticos, a técnica precisa selecionar a combinação anodo-filtro e a tensão de aceleração de elétrons para a realização do exame. O equipamento GE 700 T só apresenta uma combinação anodo-filtro, porém o Siemens apresenta três combinações anodofiltro, portanto é fundamental que a técnica saiba a diferença entre elas e escolha a mais adequada para o exame de cada paciente. A técnica é obrigada a escolher a tensão de aceleração de elétrons apenas visualizando e apalpando um pouco a mama, portanto a chance de selecionar uma tensão inadequada é alta. No equipamento GE DS isso não acontece, pois o sistema é totalmente automático. Não é necessário escolher nenhum parâmetro. A técnica é responsável apenas por posicionar a paciente adequadamente e obter a imagem.

Para uma mesma combinação anodo-filtro, as DEP's e DGM's obtidas pelo GE DS praticamente não aumentam à medida que a espessura da mama comprimida da paciente aumenta, elas ficam praticamente constantes para qualquer espessura. Esse resultado não é muito interessante, pois à medida que se aumenta a espessura da mama, as doses têm que aumentar um pouco, pois serão necessários fótons com energia maior para atravessar a mama mais espessa.

Uma vantagem do equipamento digital é a opção de ajustar alguns parâmetros da imagem, como contraste e brilho, não sendo necessário repetir o exame caso algum parâmetro desse não esteja em ótimas condições. O software permite a adequação da imagem no pós-processamento.

O maior problema nos sistemas écran-filme e digital se encontra no processamento da imagem. As imagens produzidas nos sistemas convencionais apresentam uma boa resolução espacial, porém perdem boa parte da qualidade em relação ao contraste durante o processo de revelação na processadora. Enquanto que os equipamentos digitais não apresentam uma resolução espacial suficiente, mas é compensada pela resolução de contraste e possuem uma limitação na quantidade de elementos detectores. 


\section{CONCLUSÕES}

Neste estudo foram avaliados três equipamentos de mamografia, dois que utilizam o sistema tela-filme, GE 700 T e Siemens Mammomat 3000 Nova e um digital, GE DS. Dentre estes estudos, encontram-se a implementação dos critérios de qualidade de imagem, índice de rejeição de imagem e estimativa de dose na entrada da pele e dose glandular média.

Os três mamógrafos apresentaram resultados de conformidade na maioria dos testes de controle de qualidade realizados pela equipe da STADI no IEE/USP. Nos requisitos em que não houve conformidade, a equipe de engenharia se encarregará de consertar imediatamente.

O mamógrafo digital apresentou resultados melhores do que os mamógrafos convencionais ao se analisar os critérios de qualidade de imagem, pois a processadora não interfere no processamento da imagem. Também apresentou o menor índice de rejeição de imagem, porém poderia apresentar um índice ainda menor se não houvesse tanta repetição de exame à procura da melhor imagem sem levar a dose em consideração.

A principal causa de rejeição de imagem nos três mamógrafos é o mau posicionamento da paciente. Portanto, isso mostra a necessidade de promover treinamentos periódicos para as técnicas.

$\mathrm{Na}$ determinação do valor da CSR para diferentes valores de tensão de aceleração de elétrons em cada combinação anodo-filtro dos três equipamentos, os resultados mostraram que à medida que se aumenta a tensão, o valor da CSR também aumenta, porém as combinações apresentam diferenças nos valores encontrados e isso pode ser explicado pelos diferentes rendimentos apresentados em cada combinação.

Para estimar a DEP foram utilizados os métodos do Guia da ANVISA $^{13}$ e do $\mathrm{ACR}^{16}$. Os diferentes métodos de Dance ${ }^{17,18}, \mathrm{Wu}^{19,20,21}$ e do TRS $457^{22}$ foram aplicados na estimativa da DGM.

Foi desenvolvido um manequim de acrílico em diferentes espessuras para medir o querma e estimar a DEP, pelo método do $\mathrm{ACR}^{16}$ e a $\mathrm{DGM}$, pelo método de $\mathrm{Wu}^{19,20,21}$. Este pode ser adotado para implementação rotineira de controle de 
qualidade, não só para avaliação de doses, mas também para avaliar compensação do sistema automático de exposição com a espessura da paciente.

Ao se comparar as doses, tanto a DEP quanto a DGM, para uma mesma espessura, nota-se que os valores se comportam praticamente da mesma maneira, tanto na projeção CC, quanto na MLO. Por outro lado, ao se analisar a dose recebida por uma mesma paciente durante a realização do exame de mamografia, percebe-se, nos métodos de Dance ${ }^{17,18}$ e $\mathrm{Wu}^{19,20,21}$ que a dose recebida na projeção MLO é, na maioria das vezes, maior que na projeção CC. Isso é explicado pelo fato de a espessura da mama comprimida na projeção MLO ser maior $\pm 0,5 \mathrm{~cm}$ do que na projeção CC.

Os resultados de DEP estimados pelo método do Guia da ANVISA ${ }^{13}$ são, na maioria, maiores que os estimados pelo método do $\mathrm{ACR}^{16}$ nas duas projeções estudadas em todas as combinações de todos os mamógrafos. Este resultado pode ser explicado pela aplicação do fator de correção nos valores obtidos por meio do método da ANVISA $^{13}$.

O valores de DEP estimados pelo método do Guia da ANVISA $^{13}$ nos equipamentos GE 700T e Siemens ultrapassam o nível de referência em ambas as projeções, enquanto que os valores obtidos no GE DS se encontram abaixo do nível de referência. $\mathrm{O}$ Guia do $\mathrm{ACR}^{16}$ não apresenta valores de nível de referência, portanto não é possível realizar esta análise.

Neste estudo foi realizada uma extrapolação nos grupos definidos por Dance ${ }^{18}$ para a determinação da glandularidade, que é utilizada na estimativa da DGM pelos métodos de Dance ${ }^{18}$ e do TRS $457^{22}$.

Em todas as combinações anodo-filtro dos três equipamentos, os valores estimados de DGM pelo método de Dance ${ }^{17,18}$ são maiores que os estimados pelo método de $\mathrm{Wu}^{19,20,21}$ na maioria das espessuras estudadas. Isso pode ser justificado também pelo fato da aplicação do fator de correção para o retroespalhamento na estimativa da dose por meio do método de Dance ${ }^{17,18}$. Os valores de DGM estimados pelo método do TRS $457^{22}$ giram em torno dos valores estimados pelos métodos de Dance ${ }^{17,18}$ e $\mathrm{Wu}^{19,20,21}$, mostrando a coerência entre os resultados de todos os métodos.

Os valores de DGM estimados pelo método de Dance ${ }^{17,18}$ no equipamento GE 700T estão abaixo do nível de referência para a espessura de $4,5 \mathrm{~cm}$ e acima para $6 \mathrm{~cm}$, em ambas as projeções e para qualquer faixa etária da paciente. No 
equipamento Siemens, para a combinação anodo-filtro Mo/Mo, os valores estimados de DGM estão acima do nível de referência nas duas espessuras analisadas, em ambas as projeções e em qualquer faixa etária da paciente. $\mathrm{Na}$ combinação Mo/Rh do Siemens, os valores estão abaixo do nível na projeção CC e acima do nível na MLO, com exceção dos valores estimados na espessura de $4,5 \mathrm{~cm}$ para mulheres com idade inferior a 50 anos. Todos os valores estimados no equipamento GE DS se encontram abaixo do nível de referência para ambas as espessuras.

Analisando-se os valores estimados pelo método de $\mathrm{Wu}^{19,20,21}$ nos equipamentos GE 700T e GE DS observa-se que todos os valores se encontram abaixo do nível de referência, em ambas as espessuras. No Siemens, os valores se encontram abaixo do nível de referência na projeção CC e acima na projeção MLO.

Existem valores de DGM estimados pelo método do TRS $457^{22}$ no equipamento GE 700T acima e abaixo do nível de referência, em ambas as espessuras e para qualquer faixa etária. Na combinação anodo-filtro Mo/Mo do equipamento Siemens todos os valores estão acima do nível de referência. $\mathrm{Na}$ combinação Mo/Rh deste mesmo equipamento, em ambas as espessuras existem valores acima e abaixo do nível de referência, com exceção dos valores obtidos para a espessura de $4,5 \mathrm{~cm}$ para mulheres com idade inferior a 50 anos, que se encontram abaixo do nível. Todos os valores obtidos no equipamento GE DS estimados em ambas as espessuras, se encontram abaixo do nível de referência para qualquer faixa etária.

O mamógrafo Siemens, na maioria dos resultados, obtém valores de DEP e DGM maiores que os valores apresentados pelo GE $700 \mathrm{~T}$, na combinação anodo-filtro Mo/Mo e GE DS, na combinação anodo-filtro Mo/Rh, comparando os resultados por espessura. Essa diferença pode ser justificada pelo fato dos tubos de raios $X$ possuírem diferentes rendimentos, terem sofrido diferentes desgastes, os equipamentos possuírem tempos de uso diferentes, e, especificamente no caso do GE DS, por apresentar um sistema de aquisição de imagem diferente.

Não foi viável mostrar, em gráfico, a relação da qualidade obtida na imagem contra a dose recebida pela paciente para a realização dela, em virtude da grande quantidade de dados. Porém, percebeu-se que o equipamento que fornece a 
melhor qualidade da imagem, junto à menor dose é o GE DS, na combinação Mo/Rh para mamas mais densas e $\mathrm{Rh} / \mathrm{Rh}$ para mamas menos densas.

O equipamento digital GE DS deste departamento apresentou resultados bastante satisfatórios em todas as análises realizadas, porém não é suficiente, pois não permite que sejam realizados exames de pacientes com mamas grandes.

O ideal quando uma paciente se submete a um exame de mamografia é que o departamento possua um controle de qualidade implementado, uma boa equipe de engenharia, treinamentos periódicos para as técnicas e os médicos, para que seja realizado o diagnóstico dela da maneira mais eficaz e segura, desde a exposição dela até o laudo. 


\section{FUTUROS TRABALHOS}

7.1. Desenvolver o tipo de treinamento que deve ser fornecido às técnicas para diminuir a rejeição de imagem, principalmente para as causas de maior porcentagem.

7.2. Implementar os critérios de qualidade de imagem apresentados pela publicação da European Commission ${ }^{6}$ e estimar a dose em outras regiões do corpo.

7.3. Realizar um estudo sobre a capacidade de detecção que um serviço de mamografia possui para uma futura implementação de um programa de rastreamento pelo Ministério da Saúde. 


\section{REFERÊNCIAS BIBLIOGRÁFICAS}

MINISTÉRIO DA SAÚDE. SECRETARIA DE ATENÇÃO À SAÚDE. INSTITUTO NACIONAL DO CÂNCER. COORDENAÇÃO DE PREVENÇÃO E VIGILÂNCIA DE CÂNCER. Estimativa 2008 - Incidência de Câncer no Brasil. Rio de Janeiro, INCA, 2007.

2 MINISTÉRIO DA SAÚDE. INSTITUTO NACIONAL DO CÂNCER. Estimativa 2005: Incidência de câncer no Brasil. Rio de Janeiro, INCA, 2004.

3 TOMAL, A., POLETTI, M. E., CALDAS, L. V. E. Evaluation of subject contrast and normalized average glandular dose by semi-analytical models. Applied Radiation and Isotopes, 2009. Aceito para publicação.

4 NATIONAL COUNCIL ON RADIATION PROTECTION AND MEASUREMENTS. A Guide to Mammography and Other Breast Imaging Procedures., NCRP, Report No. 149, Bethesda, MD, USA, 2004.

5 MINISTÉRIO DA SAÚDE. Diretrizes de proteção radiológica em radiodiagnóstico médico e odontológico. Brasília, Diário Oficial da União de 02 de junho de 1998 (PORTARIA DA SECRETARIA DE VIGILÂNCIA SANITÁRIA À SAÚDE 453).

${ }^{6}$ EUROPEAN COMMISSION. European guidelines on quality criteria for diagnostic radiographic images. EUR 16260 (Brussels: EC) (1996).

7 RAINFORD, L.A., AL-QATTAN, E., MCFADDEN, S., BRENNAN, P.C., CEC Analysis of radiological images produced in Europe and Asia. Science Direct, p. 1-8, 2006.

8 FURQUIM, T. A. C. Metodologia para correlação entre doses e detectabilidade em imagens mamográficas padrões: aplicação no Estado de São Paulo. 2005. Tese (Doutorado) - Instituto de Pesquisas Energéticas e Nucleares, Universidade de São Paulo, São Paulo.

9 CAROLL, Q. B. Fuch's radiographic exposure, processing and quality control. $6^{\text {th }}$ ed. Springfield, Charles C Thomas Publisher, Ltd., 1998.

${ }^{10}$ BASSETT, L. W., GOLD, R. H., KIMME-SMITH, C. History of the technical development of mammography. In: HAUS, A. G., YAFFE, M. J. Syllabus: $\boldsymbol{A}$ cathegorical course in physics. Technical aspects of breast imaging. 3..ed. Oak Brook: Radiological Society of North America (RSNA), 1994.

${ }^{11}$ THILANDER-KLANG, A. C., ACKERHOLM, P. H. R., BERLIN, I. C., BJURSTAM, N. G., MATTSSON, S. L. J., MÅNSSON, L. G., SCHÉELE, C., THUNBERG, S. J. Influence of anode-filter combinations on image quality and radiation dose in 965 women undergoing mammography. Radiology, v. 203, p. 348-354, 1997. 
${ }^{12}$ MOWLAVI, A. A. X-ray spectra calculation for different target-filter of mammograms using MCNP Code. Iranian Journal of Radiation Research. v. 3, p. 129-133, 2005.

${ }^{13}$ AGÊNCIA NACIONAL DE VIGILÂNCIA SANITÁRIA. Radiodiagnóstico médico - Segurança e Desempenho de Equipamentos. ANVISA, Brasília, 2005.

${ }^{14}$ BUSHBERG, J. T., SEIBERT, J. A., LEIDHOLDT, E. M, BOONE, J. M. The essential physics of medical imaging. Lippincott Williams \& Wilkins, Philadelphia, USA, 2002.

${ }^{15}$ KRAMER, R., DREXLER, G., PETOUSSI-HENSS, N., ZANKL, M., REGULLA, D., PANZER, W. Backscatter factors for mammography calculated with Monte Carlo methods. Physics in Medicine and Biology. v. 46, p. 771-781, 2001.

${ }^{16}$ AMERICAN COLLEGE OF RADIOLOGY. Mammography quality control manual. ACR - Committee on Quality Assurance in Mammography, 1999.

17 DANCE, D. R., Monte Carlo calculation of conversion factors for the estimation of mean glandular braest dose. Physics in Medicine and Biology, v.35, n.9, p. 1211-1219, 1990.

${ }^{18}$ DANCE, D. R., SKINNER, C. L., YOUNG, K. C., BECKETT, J. R., KOTRE, C. $J$. Additional factors for the estimation of mean glandular breast dose using the UK mammography dosimetry protocol. Physics in Medicine and Biology, v. 45, p. 3225-3240, 2000.

${ }^{19}$ WU, X., BARNES, G.T., TUCKER, D. M. Spectral dependence of tissue glandular dose in screen-film mammography. Radiology, v. 179, p. 143-148, 1991.

${ }^{20}$ WU, X., GINGOLD, E. L., BARNES, G. T., TUCKER, D. M. Normalized average glandular dose in molybdenum target-rhodium filter and rhodium target-rhodium filter mammography. Radiology, v. 193, p. 83-89, 1994.

${ }^{21}$ SOBOL, W. T., WU, X. Parametrization of mammography normalized average glandular dose tables. Medical Physics, v. 24, n. 4, p. 547-554, 1997.

22 INTERNATIONAL ATOMIC ENERGY AGENCY. Dosimetry in Diagnostic Radiology: An International Code of Practice., TRS 457, Vienna, 2007.

${ }^{23}$ KOPANS, D. B. Breast Imaging. 2. ed. Lippincott-Raven Publishers, Philadelphia, 1998.

${ }^{24}$ FREITAS, M. B. Panorama das exposições médicas em radiologia convencional no Estado de São Paulo. 2005. Tese (Doutorado) - Instituto de Física da Universidade de São Paulo, São Paulo.

${ }^{25}$ CLARKE, G. M., COOKE, D. A basic course in statistics. 2a. ed, Edward Arnold Publishers Ltd, Baltimore, USA, 1988. 
${ }^{26}$ EUROPEAN COMMISSION. European guidelines for quality assurance in breast cancer screening and diagnosis. (Brussels: EC) (2006). 